\title{
11. LATE QUATERNARY PALEOENVIRONMENTAL CHANGE ON THE NORTHEAST AUSTRALIAN MARGIN AS EVIDENCED IN OXYGEN ISOTOPE STRATIGRAPHY, MINERAL MAGNETISM, AND SEDIMENTOLOGY ${ }^{1}$
}

\author{
Ian Alexander, ${ }^{2}$ Dick Kroon, ${ }^{2}$ and Roy Thompson ${ }^{2}$
}

\begin{abstract}
To investigate late Quaternary paleoclimatic and paleoceanographic change in the sedimentary record, preserved on the Australian Continental Margin during the late Quaternary, core material was collected from Ocean Drilling Program, Leg 133, Site 819. An expanded sequence of late Quaternary, rhythmically bedded, predominantly hemipelagic sediments were recovered from Hole 819A. The foraminiferal $\delta^{18} \mathrm{O}$ record preserved at Hole $819 \mathrm{~A}$ suggests that the late Quaternary section is incomplete. Both benthic and planktonic $\delta^{18} \mathrm{O}$ stratigraphies can be traced tentatively downcore to stage 9 at about $32.5 \mathrm{mbsf}$, where a major hiatus occurs. At this level, a slump detachment surface has been identified (Shipboard Scientific Party, 1991). This slump has removed marine oxygen isotope stages 10 to 13 . Below $32.5 \mathrm{mbsf}$, continuous correlation can be achieved in the planktonic $\delta^{18} \mathrm{O}$ curve, with existing deep-sea foraminiferal oxygen isotope stratigraphies from stage 14 through stage 28.

The major hiatus at 32.5 mbsf marks the position of a significant change in the character of the sedimentation at Site 819 . Sediments below $32.5 \mathrm{mbsf}$, relative to those above $32.5 \mathrm{mbsf}$, are characterized by less variation in mean particle size; lower percentages of carbonate content in the coarse fraction $(>63 \mu \mathrm{m})$; a stronger relationship between the percentage of fine fraction and magnetic mineral concentration, and lower foraminiferal abundances. Above the hiatus, large fluctuations in mean particle size occurred, which have been interpreted to be the result of high foraminiferal abundances. Early highstands show high terrigenous influx in the fine fraction above the hiatus. This is the opposite of the general idea of high terrigenous influx during lowstands of sea level on siliciclastic dominated continental margins. We are far from understanding the origin of this material and further investigation will be required (see also Glenn et al., this volume).

All our records, except the planktonic foraminiferal oxygen isotope record, indicate that the major hiatus marks the position of a significant change in the environment at Site 819. The planktonic foraminiferal $\delta^{18} \mathrm{O}$ record suggests that environmental change occurred prior to the formation of the hiatus (i.e., near the Brunhes/Matuyama $[\mathrm{B} / \mathrm{M}]$ boundary). The interval between the $\mathrm{B} / \mathrm{M}$ boundary and the hiatus represents a transitional period between two different patterns of ocean circulation.

Throughout most of the lower part of the sequence, Site 819 was at a shallow-water depth and local oceanographic conditions were dominated by sluggish Subtropical Central Water (SCW) flow. However, near the B/M boundary, ocean circulation patterns intensified, reflecting a worldwide change in paleoenvironment. Enhanced ocean circulation patterns were possibly aided by tectonic subsidence. During this period Site 819 became progressively more under the influence of Antarctic Intermediate Water (AAIW), than SCW.

In the upper part of the sequence at Hole 819A, we see a continuation of the pattern of oceanographic reorganization suggested during stages 21 through 14. Intensification of the subsurface oceanographic circulation was also accompanied by the progressive wedging southward of surface waters associated with the East Australian Current (EAC).

The change in the nature of the records in the lower and upper parts of the sequence at Site 819 are thought to reflect perturbations by the orbital eccentricity cycle.
\end{abstract}

\section{INTRODUCTION}

The region offshore of the Australian continental margin is an extremely complex product of rifting, seafloor spreading, and margin accretion (Shipboard Scientific Party, 1991). During the evolution of the continental margin patterns of sediment production, transport and deposition have changed in response to fluctuations in both climatic and ocean circulation. The modern day physiographical setting on the continental margin, east of Cairns, is dominated by the presence of extensive carbonate bioherms (Davies et al., 1989; Harris et al., 1990). However, prior to the development of carbonate builds patterns of sediment production, transport and deposition, were most likely different and more open to the influence of ocean circulation pattern and sea level change.

To investigate the potential paleoclimatic and paleoceanographic change on the Australian Continental Margin, core material was collected from Ocean Drilling Program (ODP) Leg 133 Site 819 (NE-3). Site 819 forms part of an eastward-extending transect from the outer

\footnotetext{
'McKenzie, J.A., Davies, P.J., Palmer-Julson, A., et al., 1993. Proc. ODP, Sci. Results, 133: College Station, TX (Ocean Drilling Program).

${ }^{2}$ Department of Geology and Geophysics, University of Edinburgh, West Mains Road, Edinburgh EH9 3JW, Scotland.
}

shelf and slope of the continental margin (Sites 819 through 822) into the Queensland Trough (Site 823) and onto the Queensland Plateau (Sites 811/825 and 824) (Shipboard Scientific Party, 1991). This transect was designed to investigate the evolution of a basin and its adjacent platforms, with emphasis on developing sedimentary sequences.

\section{Site Description}

Site 819 (NE-3), drilled during Leg 133, is located in $565.2 \mathrm{~m}$ of water in Grafton Passage on the upper slope off the Great Barrier Reef (GBR) east of Cairns (Fig. 1). Sediments were recovered from Site 819 using an advanced hydraulic piston corer (APC) between 0-123 meters below seafloor (mbsf) and by the use of an extended piston core barrel (XCB) to $123-400 \mathrm{mbsf}$. Above $123 \mathrm{mbsf}, 133.5 \mathrm{~m}$ of sediment was recovered (gas expansion of cored sediments gave $108.6 \%$ recovery) at Site 819 . Below $123 \mathrm{mbsf}, 277 \mathrm{~m}$ of sediment was cored, with a $205.5-\mathrm{m}(74.3 \%)$ recovery.

Site 819 yielded an expanded Pleistocene section (spanning $<1.48$ Ma) of rhythmically bedded, hemipelagic, carbonate and siliciclastic sand and mud. Five major sedimentary units were identified by the Shipboard Scientific Party (1991), of which only the upper two fall within the scope of this study. Sedimentary Unit I, representing Cores $133-819 \mathrm{~A}-1 \mathrm{H}$ through to $-4 \mathrm{H}-3$ (0-32.5 mbsf), were found to consist 


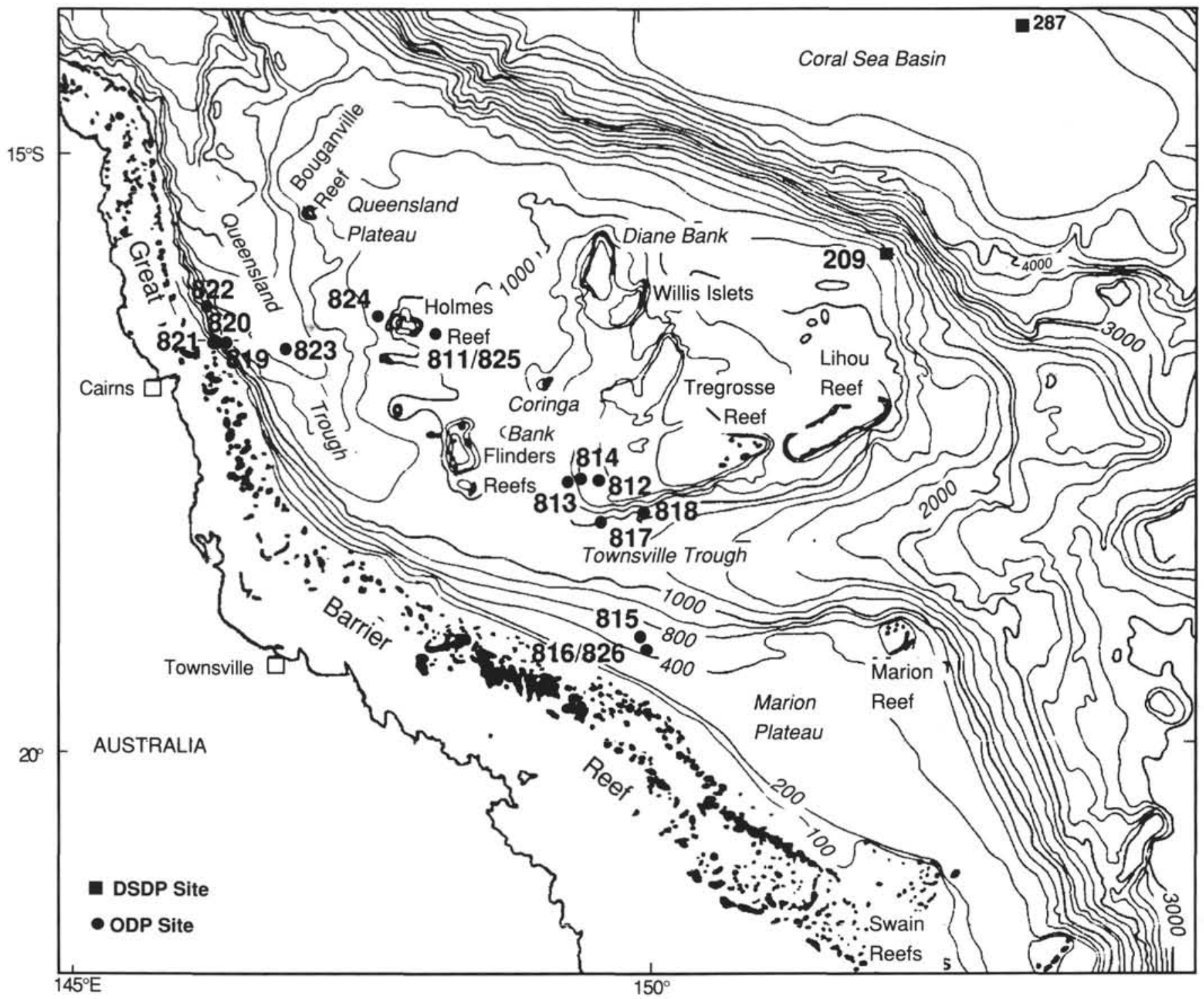

Figure 1. Map of northeastern Australian margin showing Leg 133 drill sites. Bathymetry in meters.

of rhythmically bedded sediment couplets about $9.6 \mathrm{~m}$ long; the upper half-couplet consisting of light greenish-gray clayey nannofossil ooze with assorted shallow-water bioclasts and the lower half-couplet made up of dark greenish gray clayey nannofossil ooze with numerous silt intercalations. Unit II, Cores 133-819A-4H-3 through to - 11H-2 (32.5$97 \mathrm{mbsf}$ ), made up five rhythmic couplets approximately $13 \mathrm{~m}$ thick. The lower half-couplets, in Unit II, were characterized by the presence of abundant intercalations of silt; these graded upward into the dolomitized clayey nannofossil oozes of the upper half-couplet. A stratigraphic hiatus separates sedimentary Unit I from Unit II and was visually characterized by steeply inclined bedding and a rapid change in contents of calcium carbonate (Shipboard Scientific Party, 1991).

The general depositional setting for all five sedimentary units has been interpreted as being that of an upper-slope environment, perhaps distally tapping fluviodeltaic terrigenous sources (Shipboard Scientific Party, 1991). Benthic foraminiferal assemblages indicate an upper bathyal paleodepth $(200-600 \mathrm{~m})$ for Hole $819 \mathrm{~A}$. Tentative interpretation of the sedimentary couplets suggests that the basal clay-rich half-couplets of each cycle may relate to periods of sea level lowstand and enhanced terrigenous influx, while coarser upper portions are related to periods of sea level highstand with enhanced neritic carbonate production and detrital shedding onto the slope (Shipboard Scientific Party, 1991).

\section{ANALYTICAL TECHNIQUES}

Shipboard samples were collected routinely at $20 \mathrm{~cm}$ intervals down the core to a depth of 400 mbsf. Samples from the uppermost $120 \mathrm{mbsf}$ at Hole $819 \mathrm{~A}$ form the basis of this study. Post-cruise (laboratory-based) measurements on discrete samples included magnetic susceptibility and laboratory-induced remanences, particle size characteristics, planktonic and benthic foraminiferal oxygen isotope analysis, and carbonate content determination. With the exception of benthic foraminiferal oxygen isotope analysis, all parameters were successfully completed downcore to $120 \mathrm{mbsf}$.

\section{Magnetic Mineralogy}

Five different types of laboratory-induced magnetization were employed to ascertain the likely composition and grain size of the assemblage of magnetic minerals in the Hole $819 \mathrm{~A}$ sediments. Since single magnetic parameters, such as susceptibility $(X)$, anhysteretic, 
and isothermal remanent magnetization (ARM and IRM, respectively) are largely dependent on the concentration of magnetic minerals present in a sample, several "interparametric" ratios (e.g., S ratio [Bloemendal, 1983], ARM/X, and ARM/SIRM) were determined to investigate changes in magnetic mineralogy (Kent, 1982; Robinson, 1986).

\section{Specific Magnetic Susceptibility $(X)$}

Susceptibility is a measure of the ease with which a sample can be magnetized. It is primarily dependent on the concentration of magnetic minerals present in a sample, such as magnetite and maghemite (Thompson, 1979), although it is also related to other parameters, such as magnetic grain size and shape.

Susceptibility measurements were taken on $10 \mathrm{~cm}^{3}$ sample core plugs using a Bartington Instruments susceptibility meter. Calibration was achieved using a paramagnetic salt $\left(\mathrm{FeSO}_{4} \cdot 7 \mathrm{H}_{2} \mathrm{O}\right)$ standard. Noise levels were below $0.110^{-6} \mathrm{~m}^{3} \mathrm{~kg}^{-1}$. Mass specific susceptibility measurements were obtained for samples by dividing the total susceptibility by the sample weight and expressed in units of $10^{-6} \mathrm{~m}^{3} \mathrm{~kg}^{-1}$ (Table 1).

\section{Anhysteretic Remanent Magnetization (ARM)}

Anhysteretic remanence is a laboratory-induced magnetization acquired when a sample is subjected to a declining alternating field in the presence of a direct magnetic field. The magnitude of the remanence depends on the strength of the direct magnetic field, the amplitude of the alternating magnetic field, and the susceptibility of the sample (Tarling, 1983).

ARM growth was performed using a modified Molyneux, variactype demagnetizer. Sample magnetic moments produced by the ARM growth were measured using a slow-spin Molspin fluxgate magnetometer. Results have been expressed as the square root of the sum of squares of the instrument readings (two orthogonal magnetic moments) divided by sample weight (Table 1) and are in $10^{-3} \mathrm{Am}^{2} \mathrm{~kg}^{-1}$ units. Calibration of the magnetometer was achieved using a strip of magnetic tape of known magnetic moment.

\section{Isothermal Remanent Magnetization (IRM)}

Isothermal remanence is a remanent magnetization acquired by the application of steady magnetic fields at a constant temperature, for a few seconds (Tarling, 1983). By increasing the magnetic field strength, eventually a point will be reached beyond which the remanent magnetization of the sample cannot be increased. The resulting magnetization is conventionally referred to as the saturation isothermal remanent magnetization (SIRM), although it is recognized that hematite and goethite minerals will not be strictly saturated even in high laboratory fields (Tarling, 1983).

A high-powered electromagnet was used to generate the magnetic fields for producing IRMs. Field strengths of 40,100 , and $1000 \mathrm{Mt}$ were used in our IRM acquisition work. Sample IRMs, were measured using the Molspin fluxgate magnetometer, in the same manner as outlined above for ARMs. The data obtained were divided by individual sample weights to give mass specific results, expressed in $10^{-3}$ $\mathrm{A}^{2} \mathrm{~kg}^{-1}$ (Table 1). Ratios of IRM acquired by samples in different magnetic fields, such as the S-ratio (Bloemendal, 1983), are mainly influenced by changes in the mineralogical composition of a sediments magnetic assemblage, especially the proportion of ferrimagnetic (magnetite-type) to imperfect antiferromagnetic (hematite-type) particles.

\section{Particle Size Characteristics}

Discrete sediment samples (taken from shipboard cores) were oven-dried at $60^{\circ} \mathrm{C}$, for $24 \mathrm{hr}$, weighed, and a $0.5 \mathrm{~g}$ subsample taken from each. Samples were then wet-sieved through a No. 250 mesh (63 $\mu \mathrm{m})$ stainless steel sieve. Both sediment fractions fine $(<63 \mu \mathrm{m})$ and coarse $(>63 \mu \mathrm{m})$ were retained, oven-dried at $60^{\circ} \mathrm{C}$, and later weighed to five decimal places. Fine fraction and coarse fraction dry weights were then expressed as percentages of the original dry sample weight.

Subsamples were analyzed for particle size variations using a Coulter LS-100 particle size analyzer. This instrument uses the principle of Fraunhofer diffraction of laser light in which light falling on suspended sediment particles is deflected (diffracted) by an amount that depends on the size of the particle (Agrawal et al., 1991). Subsamples were allowed to soak overnight in water, facilitating deflocculation. After a visual check for good dispersion, the samples were wet-sieved through a mesh $32(500 \mu \mathrm{m})$ stainless steel sieve, and the suspension retained. Sample volume was then made up, with water, to approximately $150 \mathrm{~mL}$. During the sieving procedure, scatted shell fragments greater than the upper running limit of the Coulter LS-100 particle size analyzer (i.e., $800 \mu \mathrm{m}$ ) were found in some of the samples (these samples were not included in the calculation of mean particle size). Samples were further disaggregated with ultrasound for $3 \mathrm{~min}$ before being run through the instrument.

\section{Oxygen Isotope Analysis}

The coarse sediment sample fraction was dry-sieved through mesh No. $45(355 \mu \mathrm{m})$ and mesh No. $60(250 \mu \mathrm{m})$ stainless steel sieves. Tests of the foraminifer species Globigerinoides ruber (planktonic) and Cibicidoides spp. (benthic) were hand-picked under a binocular microscope from the 250 - to $355-\mu$ m sediment fraction (Table 2). Care was taken to select only complete and undamaged foraminiferal tests. After selection, tests were soaked in methyl alcohol (analytical reagent) for several minutes and later cleaned in an ultrasonic bath to remove adherent contaminants. Following ultrasonic cleaning, excess methyl alcohol was drawn off with tissue paper and any residual alcohol allowed to evaporate. Foraminiferal sample weights were typically $<0.1 \mathrm{mg}$ and composed of between five to eight planktonic specimens and between two to four benthic specimens.

Following cleaning, foraminiferal tests were reacted in orthophosphoric acid (specific gravity, 1.9) at $90^{\circ} \mathrm{C}$, and the resulting $\mathrm{CO}_{2}$ gas analyzed using a VG Isogas Prism mass spectrometer. Foraminiferal samples were analyzed using a standard marble reference (SM1), and values thus obtained were converted to a PDB standard (Craig, 1957). Precision for oxygen isotope analysis was $0.085 \%$ (standard deviation for 100 analyses of a standard carbonate (SM1) conducted over several months) using SM1 sample weights of between 0.05 and 0.1 $\mathrm{mg}$. Standard deviations for duplicate samples gave typical ranges from 0.015 to 0.275 for planktonic oxygen isotope ratios and 0.093 to 0.250 for benthic oxygen isotope ratios.

\section{Carbonate Content Determination}

Percentages of carbonate contents were found using dried, $0.5-\mathrm{g}$ subsamples of the washed coarse fraction and on archive (unprocessed) sediment samples. We used the acid-base titration method to determine carbonate content, as described by Grimaldi et al. (1966).

\section{RESULTS}

\section{Oxygen Isotope Stratigraphy}

An attempt was made to correlate the foraminiferal oxygen isotope stratigraphy of Hole $819 \mathrm{~A}$ with the stacked record of Raymo et al. (1990). However, isotopic stage recognition and assignment has proved difficult, given the incomplete nature of the isotopic record at Hole 819A. Therefore, our presented isotopic stage assignment (Figs. 2 and 3 , Table 3 ) is only tentative and requires further corroboration, possibly from the isotopic stratigraphies of Holes 821 and 820 (Barton et al.; Peerdeman et al., both this volume) and/or other "Pacific type" isotope stratigraphies. Both biostratigraphic datum and magnetostratigraphic data have been used to validate our tentative foraminiferal oxygen isotope stratigraphy. Mainly nannofossil data (Wei and 
Table 1. Magnetic susceptibility and laboratory-imparted magnetization at Hole 819A.

\begin{tabular}{|c|c|c|c|c|c|c|c|c|}
\hline $\begin{array}{l}\text { Depth } \\
\text { (mbsf) }\end{array}$ & $\begin{array}{c}X \\
\left(\mu \mathrm{m}^{3} \mathrm{~kg}^{-1}\right)\end{array}$ & $\begin{array}{c}\text { ARM } \\
\left(\mathrm{mAm}^{3} \mathrm{~kg}^{-1}\right)\end{array}$ & $\begin{array}{c}\text { IRM } \\
(40 \mathrm{mT}) \\
\left(\mathrm{mAm}^{3} \mathrm{~kg}^{-1}\right)\end{array}$ & $\begin{array}{c}\text { IRM } \\
(100 \mathrm{mT}) \\
\left(\mathrm{mAm}^{3} \mathrm{~kg}^{-1}\right)\end{array}$ & $\begin{array}{c}\text { SIRM } \\
\left(\mathrm{mAm}^{3} \mathrm{~kg}^{-1}\right)\end{array}$ & $\begin{array}{c}\mathrm{ARM} / X \\
\left(\mathrm{~K} \mathrm{Am}^{-1}\right)\end{array}$ & $\begin{array}{l}\mathrm{SIRM} / X \\
\left(\mathrm{~K} \mathrm{Am}^{-1}\right)\end{array}$ & $S$ ratio \\
\hline 0.16 & 0.0055 & 3.85 & 5.26 & 8.78 & 10.05 & 7.03 & 18.36 & 0.87 \\
\hline 0.3 & 0.0058 & 4.32 & 5.22 & 8.99 & 9.58 & 7.48 & 16.58 & 0.94 \\
\hline 0.54 & 0.0048 & 4.48 & 5.40 & 9.38 & 10.27 & 9.24 & 21.19 & 0.91 \\
\hline 0.7 & 0.0052 & 4.25 & 5.66 & 9.61 & 10.35 & 8.16 & 19.87 & 0.93 \\
\hline 0.9 & 0.0054 & 4.30 & 5.53 & 9.49 & 10.75 & 7.94 & 19.84 & 0.88 \\
\hline 1.2 & 0.0068 & 4.45 & 6.01 & 10.21 & 11.13 & 6.54 & 16.35 & 0.92 \\
\hline 1.6 & 0.0059 & 4.32 & 5.35 & 8.87 & 10.07 & 7.27 & 16.93 & 0.88 \\
\hline 1.8 & 0.0047 & 3.38 & 4.56 & 7.33 & 8.31 & 7.22 & 17.73 & 0.88 \\
\hline 2.04 & 0.0057 & 4.62 & 6.43 & 10.80 & 12.31 & 8.11 & 21.58 & 0.88 \\
\hline 2.18 & 0.0063 & 5.33 & 6.69 & 11.29 & 12.12 & 8.50 & 19.31 & 0.93 \\
\hline 2.2 & 0.0069 & 5.49 & 6.48 & 10.84 & 12.18 & 7.98 & 17.71 & 0.89 \\
\hline 2.4 & 0.0070 & 5.94 & 7.06 & 12.25 & 13.28 & 8.43 & 18.84 & 0.92 \\
\hline 2.7 & 0.0081 & 6.17 & 7.59 & 13.46 & 14.72 & 7.59 & 18.12 & 0.91 \\
\hline 3.1 & 0.0080 & 5.24 & 6.79 & 11.99 & 13.61 & 6.53 & 16.95 & 0.88 \\
\hline 3.3 & 0.0081 & 5.49 & 7.57 & 13.20 & 15.03 & 6.75 & 18.48 & 0.88 \\
\hline 3.54 & 0.0068 & 5.75 & 7.41 & 12.68 & 14.10 & 8.41 & 20.64 & 0.90 \\
\hline 3.7 & 0.0080 & 6.06 & 7.69 & 12.85 & 13.04 & 7.59 & 16.33 & 0.99 \\
\hline 3.95 & 0.0092 & 5.92 & 8.21 & 12.99 & 15.04 & 6.40 & 16.27 & 0.86 \\
\hline 4.2 & 0.0104 & 5.24 & 7.74 & 12.46 & 13.78 & 5.06 & 13.30 & 0.90 \\
\hline 4.6 & 0.0127 & 6.25 & 9.47 & 14.72 & 16.24 & 4.91 & 12.77 & 0.91 \\
\hline 4.8 & 0.0135 & 5.88 & 9.43 & 13.99 & 15.29 & 4.35 & 11.31 & 0.92 \\
\hline 5.04 & 0.0208 & 6.72 & 13.52 & 17.39 & 19.06 & 3.24 & 9.18 & 0.91 \\
\hline 5.2 & 0.0222 & 6.98 & 13.55 & 17.81 & 19.63 & 3.15 & 8.85 & 0.91 \\
\hline 5.4 & 0.0178 & 6.52 & 12.60 & 17.05 & 17.93 & 3.66 & 10.07 & 0.95 \\
\hline 5.7 & 0.0213 & 5.33 & 13.74 & 18.09 & 19.76 & 2.50 & 9.26 & 0.92 \\
\hline 6.1 & 0.0385 & 3.77 & 23.88 & 35.13 & 40.75 & 0.98 & 10.60 & 0.86 \\
\hline 6.3 & 0.0293 & 2.95 & 20.64 & 27.35 & 29.92 & 1.01 & 10.21 & 0.91 \\
\hline 6.7 & 0.0078 & 1.81 & 5.07 & 7.60 & 8.69 & 2.33 & 11.21 & 0.87 \\
\hline 6.9 & 0.0068 & 2.38 & 5.33 & 8.38 & 9.24 & 3.51 & 13.59 & 0.91 \\
\hline 7.2 & 0.0031 & 2.05 & 2.81 & 5.10 & 5.56 & 6.51 & 17.67 & 0.92 \\
\hline 7.6 & 0.0041 & 2.71 & 3.63 & 5.71 & 6.14 & 6.64 & 15.05 & 0.93 \\
\hline 7.8 & 0.0018 & 0.08 & 0.38 & 0.64 & 0.97 & 0.42 & 5.38 & 0.66 \\
\hline 8.04 & 0.0022 & 0.10 & 0.52 & 0.86 & 1.21 & 0.48 & 5.61 & 0.71 \\
\hline 8.18 & 0.0018 & 0.07 & 0.39 & 0.67 & 1.02 & 0.42 & 5.80 & 0.66 \\
\hline 8.62 & 0.0020 & 0.12 & 0.53 & 0.86 & 1.22 & 0.61 & 6.16 & 0.70 \\
\hline 8.8 & 0.0023 & 0.08 & 0.46 & 0.79 & 1.10 & 0.37 & 4.85 & 0.71 \\
\hline 9.04 & 0.0032 & 0.09 & 0.55 & 0.92 & 1.24 & 0.27 & 3.88 & 0.74 \\
\hline 9.2 & 0.0024 & 0.09 & 0.54 & 0.88 & 1.25 & 0.37 & 5.28 & 0.70 \\
\hline 9.38 & 0.0027 & 0.09 & 0.57 & 0.94 & 1.34 & 0.34 & 5.06 & 0.70 \\
\hline 9.59 & 0.0031 & 0.09 & 0.54 & 0.89 & 1.25 & 0.30 & 4.04 & 0.71 \\
\hline 9.82 & 0.0038 & 0.09 & 0.52 & 0.84 & 1.17 & 0.25 & 3.10 & 0.72 \\
\hline 10.12 & 0.0031 & 0.09 & 0.58 & 0.95 & 1.35 & 0.29 & 4.33 & 0.70 \\
\hline 10.3 & 0.0033 & 0.11 & 0.58 & 0.96 & 1.42 & 0.33 & 4.35 & 0.68 \\
\hline 10.54 & 0.0036 & 0.11 & 0.73 & 1.08 & 1.56 & 0.31 & 4.37 & 0.69 \\
\hline 10.7 & 0.0036 & 0.11 & 0.65 & 1.09 & 1.67 & 0.30 & 4.69 & 0.65 \\
\hline 10.88 & 0.0036 & 0.11 & 0.68 & 1.13 & 1.74 & 0.31 & 4.79 & 0.65 \\
\hline 11.09 & 0.0039 & 0.12 & 0.74 & 1.25 & 1.95 & 0.31 & 5.03 & 0.64 \\
\hline 11.32 & 0.0050 & 0.12 & 0.74 & 1.22 & 1.83 & 0.24 & 3.66 & 0.67 \\
\hline 11.8 & 0.0040 & 0.11 & 0.63 & 1.02 & 1.35 & 0.28 & 3.41 & 0.75 \\
\hline 12.04 & 0.0036 & 0.11 & 0.64 & 1.06 & 1.49 & 0.31 & 4.08 & 0.71 \\
\hline 12.2 & 0.0047 & 0.10 & 0.58 & 0.97 & 1.30 & 0.21 & 2.76 & 0.74 \\
\hline 12.38 & 0.0039 & 0.10 & 0.57 & 0.89 & 1.18 & 0.27 & 3.00 & 0.76 \\
\hline 12.59 & 0.0040 & 0.10 & 0.52 & 0.83 & 1.09 & 0.25 & 2.72 & 0.76 \\
\hline 12.82 & 0.0045 & 0.10 & 0.64 & 0.85 & 1.08 & 0.22 & 2.42 & 0.79 \\
\hline 13.12 & 0.0032 & 0.08 & 0.41 & 0.69 & 0.96 & 0.25 & 2.98 & 0.72 \\
\hline 13.3 & 0.0034 & 0.08 & 0.37 & 0.60 & 0.82 & 0.22 & 2.38 & 0.73 \\
\hline 13.54 & 0.0034 & 0.06 & 0.32 & 0.49 & 0.63 & 0.19 & 1.85 & 0.78 \\
\hline 13.7 & 0.0029 & 0.06 & 0.32 & 0.49 & 0.68 & 0.22 & 2.37 & 0.72 \\
\hline 13.88 & 0.0035 & 0.07 & 0.38 & 0.57 & 0.72 & 0.21 & 2.07 & 0.79 \\
\hline 14.09 & 0.0042 & 0.08 & 0.36 & 0.53 & 0.68 & 0.18 & 1.63 & 0.79 \\
\hline 14.34 & 0.0027 & 0.06 & 0.30 & 0.46 & 0.58 & 0.22 & 2.18 & 0.78 \\
\hline 14.62 & 0.0024 & 0.05 & 0.32 & 0.47 & 0.60 & 0.22 & 2.49 & 0.77 \\
\hline 14.8 & 0.0024 & 0.05 & 0.27 & 0.44 & 0.57 & 0.21 & 2.33 & 0.77 \\
\hline 15.04 & 0.0020 & 0.05 & 0.24 & 0.37 & 0.46 & 0.23 & 2.32 & 0.81 \\
\hline 15.2 & 0.0018 & 0.05 & 0.21 & 0.33 & 0.43 & 0.28 & 2.45 & 0.77 \\
\hline 15.38 & 0.0015 & 0.05 & 0.23 & 0.36 & 0.49 & 0.34 & 3.15 & 0.74 \\
\hline 15.59 & 0.0017 & 0.05 & 0.23 & 0.37 & 0.49 & 0.31 & 2.94 & 0.75 \\
\hline 15.82 & 0.0018 & 0.05 & 0.24 & 0.39 & 0.50 & 0.31 & 2.86 & 0.78 \\
\hline 16.12 & 0.0015 & 0.06 & 0.28 & 0.45 & 0.54 & 0.41 & 3.50 & 0.83 \\
\hline 16.3 & 0.0017 & 0.06 & 0.25 & 0.41 & 0.56 & 0.34 & 3.35 & 0.74 \\
\hline 16.54 & 0.0016 & 0.06 & 0.28 & 0.47 & 0.59 & 0.37 & 3.68 & 0.78 \\
\hline 16.7 & 0.0023 & 0.06 & 0.29 & 0.46 & 0.58 & 0.25 & 2.56 & 0.80 \\
\hline 16.88 & 0.0020 & 0.06 & 0.32 & 0.49 & 0.65 & 0.32 & 3.32 & 0.76 \\
\hline 17.09 & 0.0016 & 0.05 & 0.26 & 0.41 & 0.53 & 0.33 & 3.20 & 0.78 \\
\hline 17.32 & 0.0026 & 0.08 & 0.32 & 0.51 & 0.65 & 0.30 & 2.52 & 0.78 \\
\hline 17.57 & 0.0018 & 0.07 & 0.28 & 0.42 & 0.49 & 0.37 & 2.66 & 0.86 \\
\hline 17.77 & 0.0015 & 0.06 & 0.25 & 0.37 & 0.50 & 0.37 & 3.26 & 0.74 \\
\hline 18.04 & 0.0020 & 0.06 & 0.25 & 0.38 & 0.50 & 0.27 & 2.49 & 0.75 \\
\hline 18.1 & 0.0019 & 0.06 & 0.27 & 0.21 & 0.56 & 0.33 & 2.97 & 0.37 \\
\hline 18.3 & 0.0022 & 0.06 & 0.30 & 0.40 & 0.62 & 0.28 & 2.86 & 0.65 \\
\hline 18.52 & 0.0024 & 0.07 & 0.33 & 0.52 & 0.65 & 0.29 & 2.70 & 0.79 \\
\hline 18.7 & 0.0019 & 0.06 & 0.27 & 0.43 & 0.55 & 0.32 & 2.86 & 0.78 \\
\hline 18.9 & 0.0020 & 0.06 & 0.27 & 0.43 & 0.57 & 0.31 & 2.88 & 0.75 \\
\hline 19.09 & 0.0016 & 0.06 & 0.23 & 0.38 & 0.52 & 0.35 & 3.23 & 0.72 \\
\hline
\end{tabular}


Table 1 (continued).

\begin{tabular}{|c|c|c|c|c|c|c|c|c|}
\hline $\begin{array}{l}\text { Depth } \\
\text { (mbsf) }\end{array}$ & $\begin{array}{c}X \\
\left(\mu \mathrm{m}^{3} \mathrm{~kg}^{-1}\right)\end{array}$ & $\begin{array}{c}\mathrm{ARM} \\
\left(\mathrm{mAm}^{3} \mathrm{~kg}^{-1}\right)\end{array}$ & $\begin{array}{c}\text { IRM } \\
(40 \mathrm{mT}) \\
\left(\mathrm{mAm}^{3} \mathrm{~kg}^{-1}\right)\end{array}$ & $\begin{array}{c}\text { IRM } \\
(100 \mathrm{mT}) \\
\left(\mathrm{mAm}^{3} \mathrm{~kg}^{-1}\right)\end{array}$ & $\begin{array}{c}\text { SIRM } \\
\left(\mathrm{mAm}^{3} \mathrm{~kg}^{-1}\right)\end{array}$ & $\begin{array}{c}\mathrm{ARM} / X \\
\left(\mathrm{~K} \mathrm{Am}^{-1}\right)\end{array}$ & $\begin{array}{c}\mathrm{SIRM} / X \\
\left(\mathrm{~K} \mathrm{Am}^{-1}\right)\end{array}$ & $S$ ratio \\
\hline 19.31 & 0.0016 & 0.06 & 0.23 & 0.37 & 0.50 & 0.35 & 3.10 & 0.75 \\
\hline 19.6 & 0.0013 & 0.05 & 0.20 & 0.34 & 0.48 & 0.42 & 3.67 & 0.71 \\
\hline 19.8 & 0.0017 & 0.06 & 0.21 & 0.35 & 0.48 & 0.32 & 2.82 & 0.74 \\
\hline 20.02 & 0.0016 & 0.08 & 0.31 & 0.56 & 0.76 & 0.53 & 4.83 & 0.74 \\
\hline 20.2 & 0.0017 & 0.07 & 0.37 & 0.60 & 0.79 & 0.41 & 4.63 & 0.77 \\
\hline 20.4 & 0.0019 & 0.09 & 0.44 & 0.72 & 0.98 & 0.45 & 5.12 & 0.74 \\
\hline 20.59 & 0.0021 & 0.08 & 0.47 & 0.78 & 1.08 & 0.40 & 5.23 & 0.72 \\
\hline 20.81 & 0.0024 & 0.10 & 0.58 & 1.01 & 1.44 & 0.40 & 6.06 & 0.70 \\
\hline 21.1 & 0.0042 & 0.16 & 1.02 & 1.70 & 2.45 & 0.39 & 5.82 & 0.69 \\
\hline 21.3 & 0.0074 & 6.90 & 8.72 & 13.67 & 15.05 & 9.35 & 20.40 & 0.91 \\
\hline 21.52 & 0.0078 & 7.46 & 8.62 & 14.34 & 16.31 & 9.58 & 20.95 & 0.88 \\
\hline 21.7 & 0.0078 & 7.22 & 8.03 & 13.40 & 15.12 & 9.24 & 19.33 & 0.89 \\
\hline 21.9 & 0.0049 & 0.14 & 0.72 & 1.24 & 1.92 & 0.28 & 3.90 & 0.65 \\
\hline 22.09 & 0.0048 & 0.13 & 0.72 & 1.26 & 1.70 & 0.28 & 3.54 & 0.74 \\
\hline 22.31 & 0.0050 & 0.15 & 0.79 & 1.36 & 1.83 & 0.30 & 3.68 & 0.74 \\
\hline 22.6 & 0.0038 & 0.19 & 1.17 & 2.10 & 2.75 & 0.49 & 7.28 & 0.76 \\
\hline 22.8 & 0.0047 & 0.16 & 0.85 & 1.57 & 1.92 & 0.34 & 4.09 & 0.82 \\
\hline 23.02 & 0.0044 & 0.13 & 0.79 & 1.30 & 1.80 & 0.29 & 4.09 & 0.72 \\
\hline 23.2 & 0.0047 & 0.14 & 0.76 & 1.27 & 1.69 & 0.29 & 3.57 & 0.75 \\
\hline 23.4 & 0.0048 & 0.16 & 0.82 & 1.46 & 1.99 & 0.32 & 4.10 & 0.74 \\
\hline 23.59 & 0.0051 & 0.16 & 0.87 & 1.55 & 2.14 & 0.32 & 4.18 & 0.73 \\
\hline 23.81 & 0.0048 & 0.21 & 1.00 & 1.80 & 2.38 & 0.43 & 4.93 & 0.76 \\
\hline 24.1 & 0.0041 & 0.14 & 0.63 & 1.08 & 1.52 & 0.34 & 3.74 & 0.71 \\
\hline 24.3 & 0.0036 & 0.10 & 0.56 & 0.98 & 1.49 & 0.29 & 4.17 & 0.66 \\
\hline 24.52 & 0.0161 & 2.15 & 13.70 & 19.15 & 21.40 & 1.33 & 13.26 & 0.89 \\
\hline 24.7 & 0.0193 & 1.83 & 14.37 & 20.56 & 23.90 & 0.95 & 12.40 & 0.86 \\
\hline 24.87 & 0.0199 & 0.53 & 5.19 & 7.63 & 10.42 & 0.27 & 5.23 & 0.73 \\
\hline 25.09 & 0.0098 & 0.08 & 0.45 & 0.74 & 1.09 & 0.09 & 1.11 & 0.67 \\
\hline 25.57 & 0.0016 & 0.00 & 0.00 & 0.00 & 0.00 & 0.00 & 0.00 & \\
\hline 25.76 & 0.0010 & 0.06 & 0.27 & 0.51 & 0.75 & 0.56 & 7.28 & 0.67 \\
\hline 26.02 & 0.0026 & 0.11 & 0.62 & 1.11 & 1.74 & 0.43 & 6.65 & 0.64 \\
\hline 26.17 & 0.0030 & 0.14 & 0.81 & 1.37 & 1.97 & 0.48 & 6.51 & 0.70 \\
\hline 26.4 & 0.0030 & 0.13 & 0.76 & 1.29 & 1.93 & 0.42 & 6.46 & 0.67 \\
\hline 26.59 & 0.0016 & 0.09 & 0.44 & 0.74 & 1.06 & 0.55 & 6.75 & 0.70 \\
\hline 26.81 & 0.0010 & 0.05 & 0.23 & 0.37 & 0.50 & 0.54 & 5.12 & 0.74 \\
\hline 27.1 & 0.0005 & 0.05 & 0.24 & 0.40 & 0.58 & 0.87 & 10.83 & 0.70 \\
\hline 27.8 & 0.0023 & 0.07 & 0.32 & 0.50 & 0.64 & 0.32 & 2.74 & 0.79 \\
\hline 28 & 0.0017 & 0.07 & 0.35 & 0.55 & 0.72 & 0.42 & 4.30 & 0.76 \\
\hline 28.2 & 0.0043 & 0.12 & 0.59 & 0.93 & 1.13 & 0.28 & 2.64 & 0.82 \\
\hline 28.4 & 0.0046 & 0.12 & 0.57 & 0.94 & 1.15 & 0.26 & 2.51 & 0.82 \\
\hline 28.6 & 0.0034 & 0.09 & 0.49 & 0.74 & 0.90 & 0.28 & 2.66 & 0.82 \\
\hline 28.8 & 0.0020 & 0.07 & 0.50 & 0.85 & 1.11 & 0.33 & 5.47 & 0.77 \\
\hline 29.1 & 0.0007 & 0.04 & 0.33 & 0.52 & 0.65 & 0.63 & 9.87 & 0.80 \\
\hline 29.3 & 0.0005 & 0.04 & 0.21 & 0.33 & 0.44 & 0.86 & 9.44 & 0.76 \\
\hline 29.5 & 0.0005 & 0.04 & 0.19 & 0.29 & 0.40 & 0.89 & 8.86 & 0.72 \\
\hline 29.7 & 0.0006 & 0.06 & 0.25 & 0.42 & 0.55 & 1.04 & 9.74 & 0.77 \\
\hline 29.9 & 0.0012 & 0.05 & 0.22 & 0.35 & 0.45 & 0.42 & 3.65 & 0.78 \\
\hline 30.1 & 0.0016 & 0.06 & 0.23 & 0.34 & 0.47 & 0.35 & 2.98 & 0.73 \\
\hline 30.3 & 0.0013 & 0.05 & 0.19 & 0.31 & 0.39 & 0.37 & 2.98 & 0.80 \\
\hline 30.6 & 0.0010 & 0.06 & 0.23 & 0.36 & 0.48 & 0.60 & 4.75 & 0.75 \\
\hline 30.8 & 0.0013 & 0.06 & 0.25 & 0.37 & 0.49 & 0.49 & 3.81 & 0.75 \\
\hline 31 & 0.0021 & 0.06 & 0.28 & 0.43 & 0.58 & 0.26 & 2.74 & 0.74 \\
\hline 31.2 & 0.0022 & 0.06 & 0.29 & 0.46 & 0.61 & 0.28 & 2.83 & 0.76 \\
\hline 31.4 & 0.0025 & 0.06 & 0.35 & 0.54 & 0.69 & 0.26 & 2.79 & 0.78 \\
\hline 31.6 & 0.0029 & 0.08 & 0.32 & 0.50 & 0.66 & 0.27 & 2.30 & 0.76 \\
\hline 31.8 & 0.0032 & 0.09 & 0.39 & 0.62 & 0.82 & 0.28 & 2.60 & 0.76 \\
\hline 32.1 & 0.0030 & 0.08 & 0.31 & 0.48 & 0.61 & 0.25 & 2.02 & 0.80 \\
\hline 32.3 & 0.0014 & 0.06 & 0.16 & 0.27 & 0.37 & 0.41 & 2.57 & 0.74 \\
\hline 32.5 & 0.0022 & 0.07 & 0.27 & 0.41 & 0.54 & 0.30 & 2.44 & 0.76 \\
\hline 32.7 & 0.0028 & 0.08 & 0.30 & 0.48 & 0.60 & 0.27 & 2.16 & 0.79 \\
\hline 32.9 & 0.0048 & 0.14 & 1.06 & 1.72 & 1.95 & 0.28 & 4.05 & 0.88 \\
\hline 33.1 & 0.0053 & 0.15 & 0.65 & 1.16 & 1.43 & 0.29 & 2.70 & 0.81 \\
\hline 33.3 & 0.0050 & 0.12 & 0.58 & 0.94 & 1.16 & 0.24 & 2.31 & 0.81 \\
\hline 33.6 & 0.0033 & 0.11 & 0.50 & 0.90 & 1.09 & 0.34 & 3.32 & 0.82 \\
\hline 33.8 & 0.0034 & 0.12 & 0.54 & 0.91 & 1.16 & 0.35 & 3.40 & 0.78 \\
\hline 34 & 0.0031 & 0.16 & 0.61 & 1.06 & 1.32 & 0.51 & 4.24 & 0.80 \\
\hline 34.2 & 0.0034 & 0.14 & 0.63 & 1.03 & 1.24 & 0.40 & 3.61 & 0.83 \\
\hline 34.4 & 0.0036 & 0.16 & 0.63 & 1.08 & 1.31 & 0.44 & 3.66 & 0.82 \\
\hline 34.6 & 0.0032 & 0.11 & 0.42 & 0.74 & 0.92 & 0.35 & 2.85 & 0.81 \\
\hline 34.8 & 0.0042 & 0.14 & 0.54 & 0.91 & 1.12 & 0.33 & 2.69 & 0.81 \\
\hline 35.1 & 0.0028 & 0.11 & 0.49 & 0.79 & 0.97 & 0.40 & 3.50 & 0.81 \\
\hline 35.3 & 0.0031 & 0.12 & 0.49 & 0.86 & 1.10 & 0.38 & 3.50 & 0.78 \\
\hline 35.5 & 0.0033 & 0.12 & 0.49 & 0.87 & 1.01 & 0.37 & 3.09 & 0.86 \\
\hline 35.7 & 0.0029 & 0.10 & 0.42 & 0.73 & 0.93 & 0.34 & 3.25 & 0.78 \\
\hline 35.9 & 0.0031 & 0.10 & 0.50 & 0.77 & 0.98 & 0.33 & 3.20 & 0.78 \\
\hline 36.1 & 0.0031 & 0.11 & 0.55 & 0.89 & 1.10 & 0.35 & 3.58 & 0.81 \\
\hline 36.3 & 0.0032 & 0.13 & 0.59 & 0.95 & 1.16 & 0.40 & 3.61 & 0.82 \\
\hline 36.6 & 0.0031 & 0.12 & 0.47 & 0.82 & 1.00 & 0.38 & 3.25 & 0.82 \\
\hline 36.8 & 0.0030 & 0.12 & 0.43 & 0.80 & 0.99 & 0.40 & 3.33 & 0.81 \\
\hline 37 & 0.0026 & 0.11 & 0.43 & 0.80 & 0.91 & 0.42 & 3.47 & 0.89 \\
\hline 37.2 & 0.0023 & 0.11 & 0.40 & 0.67 & 0.84 & 0.48 & 3.72 & 0.79 \\
\hline 37.1 & 0.0033 & 0.11 & 0.42 & 0.77 & 0.89 & 0.34 & 2.67 & 0.86 \\
\hline 37.3 & 0.0026 & 0.10 & 0.45 & 0.79 & 0.97 & 0.40 & 3.77 & 0.81 \\
\hline 37.5 & 0.0026 & 0.11 & 0.47 & 0.82 & 1.01 & 0.42 & 3.90 & 0.81 \\
\hline
\end{tabular}


Table 1 (continued).

\begin{tabular}{|c|c|c|c|c|c|c|c|c|}
\hline $\begin{array}{l}\text { Depth } \\
\text { (mbsf) }\end{array}$ & $\begin{array}{c}X \\
\left(\mu \mathrm{m}^{3} \mathrm{~kg}^{-1}\right)\end{array}$ & $\underset{\left(\mathrm{mAm}^{3} \mathrm{~kg}^{-1}\right)}{\mathrm{ARM}}$ & $\begin{array}{c}\text { IRM } \\
(40 \mathrm{mT}) \\
\left(\mathrm{mAm}^{3} \mathrm{~kg}^{-1}\right)\end{array}$ & $\begin{array}{c}\text { IRM } \\
(100 \mathrm{mT}) \\
\left(\mathrm{mAm}^{3} \mathrm{~kg}^{-1}\right)\end{array}$ & $\underset{\left(\mathrm{mAm}^{3} \mathrm{~kg}^{-1}\right)}{\mathrm{SIRM}}$ & $\begin{array}{c}\mathrm{ARM} / \mathrm{X} \\
\left(\mathrm{K} \mathrm{Am}^{-1}\right)\end{array}$ & $\begin{array}{c}\mathrm{SIRM} / X \\
\left(\mathrm{~K} \mathrm{Am}^{-1}\right)\end{array}$ & $S$ ratio \\
\hline 37.7 & 0.0025 & 0.11 & 0.48 & 0.80 & 1.00 & 0.44 & 3.98 & 0.80 \\
\hline 37.9 & 0.0024 & 0.10 & 0.43 & 0.71 & 0.88 & 0.43 & 3.67 & 0.81 \\
\hline 38.1 & 0.0025 & 0.11 & 0.45 & 0.77 & 0.96 & 0.44 & 3.88 & 0.80 \\
\hline 38.3 & 0.0022 & 0.09 & 0.39 & 0.69 & 0.86 & 0.41 & 3.86 & 0.80 \\
\hline 38.6 & 0.0024 & 0.09 & 0.37 & 0.65 & 0.81 & 0.36 & 3.40 & 0.80 \\
\hline 38.8 & 0.0024 & 0.08 & 0.35 & 0.62 & 0.81 & 0.35 & 3.37 & 0.77 \\
\hline 39 & 0.0025 & 0.08 & 0.33 & 0.56 & 0.70 & 0.33 & 2.76 & 0.80 \\
\hline 39.2 & 0.0025 & 0.09 & 0.38 & 0.65 & 0.83 & 0.36 & 3.27 & 0.79 \\
\hline 39.4 & 0.0020 & 0.09 & 0.36 & 0.61 & 0.77 & 0.42 & 3.78 & 0.80 \\
\hline 39.6 & 0.0022 & 0.09 & 0.46 & 0.77 & 0.97 & 0.42 & 4.41 & 0.79 \\
\hline 39.8 & 0.0023 & 0.08 & 0.36 & 0.63 & 0.76 & 0.37 & 3.37 & 0.82 \\
\hline 40.1 & 0.0021 & 0.07 & 0.34 & 0.60 & 0.77 & 0.33 & 3.78 & 0.78 \\
\hline 40.3 & 0.0021 & 0.06 & 0.30 & 0.54 & 0.63 & 0.27 & 2.99 & 0.86 \\
\hline 40.5 & 0.0025 & 0.09 & 0.37 & 0.64 & 0.81 & 0.36 & 3.18 & 0.79 \\
\hline 40.7 & 0.0028 & 0.09 & 0.41 & 0.75 & 0.92 & 0.33 & 3.25 & 0.81 \\
\hline 40.9 & 0.0026 & 0.14 & 0.53 & 0.94 & 1.15 & 0.53 & 4.40 & 0.82 \\
\hline 41.1 & 0.0027 & 0.17 & 0.55 & 1.00 & 1.19 & 0.64 & 4.34 & 0.84 \\
\hline 41.3 & 0.0028 & 0.16 & 0.54 & 1.21 & 1.51 & 0.55 & 5.31 & 0.80 \\
\hline 41.6 & 0.0033 & 0.15 & 0.53 & 0.84 & 1.03 & 0.45 & 3.17 & 0.82 \\
\hline 41.8 & 0.0031 & 0.17 & 0.54 & 1.00 & 1.25 & 0.53 & 4.03 & 0.80 \\
\hline 42 & 0.0029 & 0.17 & 0.67 & 1.66 & 2.12 & 0.60 & 7.23 & 0.78 \\
\hline 42,2 & 0.0027 & 0.17 & 0.64 & 1.20 & 1.46 & 0.64 & 5.51 & 0.82 \\
\hline 42.4 & 0.0029 & 0.16 & 0.59 & 1.02 & 1.19 & 0.54 & 4.14 & 0.86 \\
\hline 42.6 & 0.0026 & 0.17 & 0.61 & 1.00 & 1.20 & 0.64 & 4.57 & 0.83 \\
\hline 42.8 & 0.0027 & 0.21 & 0.61 & 0.95 & 1.11 & 0.76 & 4.11 & 0.85 \\
\hline 43.1 & 0.0031 & 0.20 & 0.68 & 1.03 & 1.22 & 0.64 & 3.92 & 0.84 \\
\hline 43.3 & 0.0027 & 0.21 & 0.71 & 1.14 & 1.29 & 0.81 & 4.86 & 0.89 \\
\hline 43.5 & 0.0026 & 0.22 & 0.68 & 1.03 & 1.18 & 0.86 & 4.58 & 0.87 \\
\hline 43.7 & 0.0033 & 0.23 & 0.72 & 1.13 & 1.34 & 0.71 & 4.13 & 0.84 \\
\hline 43.9 & 0.0024 & 0.19 & 0.64 & 0.97 & 1.14 & 0.78 & 4.72 & 0.85 \\
\hline 44.1 & 0.0026 & 0.18 & 0.57 & 0.88 & 1.03 & 0.67 & 3.89 & 0.86 \\
\hline 44.3 & 0.0023 & 0.15 & 0.48 & 0.74 & 0.90 & 0.64 & 3.95 & 0.82 \\
\hline 44.6 & 0.0026 & 0.13 & 0.46 & 0.74 & 0.92 & 0.50 & 3.54 & 0.80 \\
\hline 44.8 & 0.0024 & 0.13 & 0.43 & 0.66 & 0.81 & 0.56 & 3.38 & 0.81 \\
\hline 45 & 0.0022 & 0.11 & 0.44 & 0.69 & 0.86 & 0.49 & 3.90 & 0.80 \\
\hline 45.2 & 0.0027 & 0.13 & 0.44 & 0.71 & 0.88 & 0.49 & 3.23 & 0.81 \\
\hline 45.4 & 0.0025 & 0.13 & 0.46 & 0.70 & 0.87 & 0.53 & 3.46 & 0.80 \\
\hline 45.6 & 0.0030 & 0.19 & 0.57 & 0.87 & 1.06 & 0.61 & 3.51 & 0.82 \\
\hline 45.8 & 0.0030 & 0.16 & 0.56 & 0.86 & 1.04 & 0.54 & 3.48 & 0.83 \\
\hline 46.1 & 0.0035 & 0.21 & 0.62 & 1.03 & 1.26 & 0.58 & 3.55 & 0.82 \\
\hline 46.3 & 0.0034 & 0.21 & 0.62 & 1.03 & 1.19 & 0.61 & 3.49 & 0.86 \\
\hline 46.5 & 0.0026 & 0.23 & 0.67 & 1.03 & 1.17 & 0.89 & 4.49 & 0.88 \\
\hline 46.7 & 0.0026 & 0.19 & 0.64 & 0.96 & 1.11 & 0.76 & 4.32 & 0.86 \\
\hline 46.6 & 0.0035 & 0.29 & 0.87 & 1.31 & 1.53 & 0.82 & 4.38 & 0.86 \\
\hline 46.8 & 0.0036 & 0.30 & 0.91 & 1.37 & 1.59 & 0.84 & 4.45 & 0.86 \\
\hline 47 & 0.0035 & 0.31 & 1.03 & 1.51 & 1.71 & 0.88 & 4.95 & 0.88 \\
\hline 47.2 & 0.0036 & 0.33 & 1.03 & 1.53 & 1.78 & 0.92 & 4.91 & 0.86 \\
\hline 47.4 & 0.0040 & 0.32 & 0.98 & 1.54 & 1.60 & 0.82 & 4.04 & 0.96 \\
\hline 47.6 & 0.0040 & 0.33 & 0.97 & 1.54 & 1.72 & 0.83 & 4.31 & 0.89 \\
\hline 47.8 & 0.0039 & 0.29 & 0.86 & 1.40 & 1.64 & 0.74 & 4.24 & 0.86 \\
\hline 48.1 & 0.0041 & 0.28 & 0.85 & 1.41 & 1.63 & 0.69 & 3.99 & 0.87 \\
\hline 48.3 & 0.0040 & 0.26 & 0.76 & 1.19 & 1.36 & 0.65 & 3.44 & 0.87 \\
\hline 48.5 & 0.0034 & 0.27 & 0.78 & 1.15 & 1.34 & 0.79 & 3.99 & 0.85 \\
\hline 48.7 & 0.0037 & 0.21 & 0.64 & 0.96 & 1.15 & 0.55 & 3.07 & 0.84 \\
\hline 48.9 & 0.0037 & 0.21 & 0.70 & 1.02 & 1.20 & 0.55 & 3.20 & 0.85 \\
\hline 49.1 & 0.0035 & 0.18 & 0.58 & 0.86 & 1.00 & 0.52 & 2.83 & 0.87 \\
\hline 49.3 & 0.0034 & 0.14 & 0.45 & 0.72 & 0.86 & 0.42 & 2.54 & 0.84 \\
\hline 49.6 & 0.0033 & 0.09 & 0.35 & 0.58 & 0.75 & 0.29 & 2.29 & 0.78 \\
\hline 49.8 & 0.0030 & 0.10 & 0.32 & 0.58 & 0.73 & 0.33 & 2.43 & 0.79 \\
\hline 50 & 0.0025 & 0.09 & 0.31 & 0.53 & 0.67 & 0.34 & 2.65 & 0.79 \\
\hline 50.2 & 0.0021 & 0.08 & 0.32 & 0.49 & 0.55 & 0.39 & 2.67 & 0.90 \\
\hline 50.4 & 0.0019 & 0.09 & 0.27 & 0.49 & 0.65 & 0.47 & 3.44 & 0.75 \\
\hline 50.6 & 0.0022 & 0.07 & 0.26 & 0.48 & 0.62 & 0.32 & 2.85 & 0.77 \\
\hline 50.8 & 0.0020 & 0.07 & 0.27 & 0.49 & 0.63 & 0.38 & 3.20 & 0.77 \\
\hline 51.1 & 0.0017 & 0.07 & 0.24 & 0.42 & 0.56 & 0.41 & 3.22 & 0.74 \\
\hline 51.3 & 0.0020 & 0.07 & 0.25 & 0.44 & 0.57 & 0.35 & 2.87 & 0.78 \\
\hline 51.5 & 0.0025 & 0.07 & 0.26 & 0.47 & 0.62 & 0.26 & 2.42 & 0.76 \\
\hline 51.7 & 0.0019 & 0.06 & 0.25 & 0.45 & 0.59 & 0.33 & 3.01 & 0.76 \\
\hline 51.9 & 0.0020 & 0.06 & 0.26 & 0.45 & 0.54 & 0.33 & 2.73 & 0.84 \\
\hline 52.1 & 0.0020 & 0.07 & 0.24 & 0.47 & 0.57 & 0.37 & 2.86 & 0.82 \\
\hline 52.3 & 0.0017 & 0.07 & 0.25 & 0.45 & 0.59 & 0.42 & 3.60 & 0.76 \\
\hline 52.6 & 0.0016 & 0.07 & 0.25 & 0.45 & 0.57 & 0.46 & 3.63 & 0.79 \\
\hline 52.8 & 0.0014 & 0.06 & 0.23 & 0.42 & 0.62 & 0.43 & 4.47 & 0.68 \\
\hline 53 & 0.0017 & 0.09 & 0.29 & 0.51 & 0.67 & 0.54 & 4.01 & 0.75 \\
\hline 53.2 & 0.0016 & 0.08 & 0.23 & 0.47 & 0.53 & 0.48 & 3.37 & 0.88 \\
\hline 53.4 & 0.0012 & 0.06 & 0.23 & 0.41 & 0.50 & 0.48 & 4.08 & 0.83 \\
\hline 53.6 & 0.0014 & 0.06 & 0.23 & 0.41 & 0.53 & 0.43 & 3.73 & $0-77$ \\
\hline 53.8 & 0.0013 & 0.06 & 0.21 & 0.39 & 0.48 & 0.44 & 3.59 & 0.82 \\
\hline 54.1 & 0.0013 & 0.05 & 0.20 & 0.38 & 0.52 & 0.36 & 3.98 & 0.73 \\
\hline 54.3 & 0.0015 & 0.08 & 0.28 & 0.51 & 0.71 & 0.51 & 4.67 & 0.72 \\
\hline 54.5 & 0.0014 & 0.07 & 0.25 & 0.43 & 0.56 & 0.55 & 4.12 & 0.77 \\
\hline 54.7 & 0.0014 & 0.07 & 0.23 & 0.42 & 0.55 & 0.50 & 3.97 & 0.76 \\
\hline 54.9 & 0.0013 & 0.06 & 0.19 & 0.36 & 0.50 & 0.48 & 3.81 & 0.72 \\
\hline 55.1 & 0.0014 & 0.08 & 0.24 & 0.45 & 0.60 & 0.57 & 4.39 & 0.75 \\
\hline
\end{tabular}


Table 1 (continued).

\begin{tabular}{|c|c|c|c|c|c|c|c|c|}
\hline $\begin{array}{l}\text { Depth } \\
\text { (mbsf) }\end{array}$ & $\begin{array}{c}X \\
\left(\mu \mathrm{m}^{3} \mathrm{~kg}^{-1}\right)\end{array}$ & $\begin{array}{c}\text { ARM } \\
\left(\mathrm{mAm}^{3} \mathrm{~kg}^{-1}\right)\end{array}$ & $\begin{array}{c}\text { IRM } \\
(40 \mathrm{mT}) \\
\left(\mathrm{mAm}^{3} \mathrm{~kg}^{-1}\right)\end{array}$ & $\begin{array}{c}\text { IRM } \\
(100 \mathrm{mT}) \\
\left(\mathrm{mAm}^{3} \mathrm{~kg}^{-1}\right)\end{array}$ & $\underset{\left(\mathrm{mAm}^{3} \mathrm{~kg}^{-1}\right)}{\text { SIRM }}$ & $\begin{array}{c}\mathrm{ARM} / \mathrm{X} \\
\left(\mathrm{K} \mathrm{Am}^{-1}\right)\end{array}$ & $\begin{array}{l}\mathrm{SIRM} / X \\
\left(\mathrm{~K} \mathrm{Am}^{-1}\right)\end{array}$ & $S$ ratio \\
\hline 55.3 & 0.0022 & 0.07 & 0.25 & 0.47 & 0.55 & 0.31 & 2.49 & 0.84 \\
\hline 56.1 & 0.0016 & 0.07 & 0.22 & 0.40 & 0.52 & 0.44 & 3.32 & 0.78 \\
\hline 56.3 & 0.0016 & 0.07 & 0.26 & 0.47 & 0.61 & 0.43 & 3.80 & 0.78 \\
\hline 56.5 & 0.0014 & 0.07 & 0.29 & 0.52 & 0.67 & 0.47 & 4.69 & 0.76 \\
\hline 56.7 & 0.0014 & 0.09 & 0.30 & 0.56 & 0.68 & 0.64 & 4.77 & 0.82 \\
\hline 56.9 & 0.0015 & 0.08 & 0.28 & 0.49 & 0.58 & 0.54 & 3.96 & 0.85 \\
\hline 57.1 & 0.0018 & 0.07 & 0.32 & 0.58 & 0.76 & 0.42 & 4.24 & 0.77 \\
\hline 57.3 & 0.0017 & 0.08 & 0.28 & 0.49 & 0.62 & 0.46 & 3.55 & 0.79 \\
\hline 57.6 & 0.0016 & 0.08 & 0.35 & 0.60 & 0.79 & 0.51 & 4.78 & 0.77 \\
\hline 57.9 & 0.0016 & 0.09 & 0.28 & 0.51 & 0.65 & 0.57 & 4.17 & 0.78 \\
\hline 58 & 0.0017 & 0.08 & 0.26 & 0.51 & 0.62 & 0.48 & 3.73 & 0.82 \\
\hline 58.2 & 0.0017 & 0.08 & 0.30 & 0.56 & 0.73 & 0.49 & 4.29 & 0.78 \\
\hline 58.4 & 0.0017 & 0.09 & 0.33 & 0.59 & 0.78 & 0.51 & 4.52 & 0.75 \\
\hline 58.6 & 0.0016 & 0.08 & 0.36 & 0.65 & 0.83 & 0.51 & 5.09 & 0.78 \\
\hline 58.8 & 0.0016 & 0.09 & 0.35 & 0.63 & 0.82 & 0.55 & 5.20 & 0.77 \\
\hline 59.1 & 0.0020 & 0.09 & 0.36 & 0.64 & 0.82 & 0.46 & 4.16 & 0.78 \\
\hline 59.3 & 0.0018 & 0.09 & 0.37 & 0.65 & 0.81 & 0.51 & 4.48 & 0.80 \\
\hline 59.5 & 0.0018 & 0.10 & 0.35 & 0.62 & 0.82 & 0.52 & 4.47 & 0.76 \\
\hline 59.7 & 0.0018 & 0.09 & 0.35 & 0.60 & 0.75 & 0.47 & 4.15 & 0.79 \\
\hline 59.9 & 0.0017 & 0.09 & 0.35 & 0.62 & 0.80 & 0.51 & 4.68 & 0.77 \\
\hline 60.1 & 0.0021 & 0.09 & 0.34 & 0.61 & 0.74 & 0.42 & 3.57 & 0.82 \\
\hline 60.3 & 0.0018 & 0.09 & 0.34 & 0.60 & 0.78 & 0.50 & 4.45 & 0.76 \\
\hline 60.6 & 0.0021 & 0.09 & 0.38 & 0.67 & 0.80 & 0.41 & 3.80 & 0.84 \\
\hline 60.8 & 0.0026 & 0.11 & 0.42 & 0.74 & 0.93 & 0.42 & 3.61 & 0.80 \\
\hline 61 & 0.0019 & 0.08 & 0.34 & 0.60 & 0.74 & 0.40 & 3.93 & 0.81 \\
\hline 61.2 & 0.0020 & 0.10 & 0.33 & 0.59 & 0.77 & 0.49 & 3.78 & 0.77 \\
\hline 61.4 & 0.0025 & 0.08 & 0.33 & 0.56 & 0.73 & 0.33 & 2.88 & 0.77 \\
\hline 61.6 & 0.0024 & 0.11 & 0.39 & 0.71 & 0.95 & 0.43 & 3.87 & 0.75 \\
\hline 61.8 & 0.0027 & 0.09 & 0.34 & 0.59 & 0.70 & 0.34 & 2.58 & 0.84 \\
\hline 62.1 & 0.0027 & 0.10 & 0.40 & 0.71 & 0.86 & 0.37 & 3.23 & 0.82 \\
\hline 62.3 & 0.0026 & 0.09 & 0.38 & 0.65 & 0.83 & 0.35 & 3.15 & 0.78 \\
\hline 62.5 & 0.0025 & 0.09 & 0.38 & 0.65 & 0.79 & 0.34 & 3.09 & 0.83 \\
\hline 62.7 & 0.0031 & 0.10 & 0.42 & 0.71 & 0.90 & 0.33 & 2.94 & 0.79 \\
\hline 62.9 & 0.0029 & 0.10 & 0.41 & 0.68 & 0.84 & 0.34 & 2.92 & 0.82 \\
\hline 63.1 & 0.0028 & 0.10 & 0.43 & 0.72 & 0.87 & 0.36 & 3.10 & 0.83 \\
\hline 63.6 & 0.0030 & 0.09 & 0.43 & 0.71 & 0.91 & 0.31 & 3.01 & 0.77 \\
\hline 63.8 & 0.0032 & 0.10 & 0.45 & 0.75 & 0.92 & 0.32 & 2.91 & 0.82 \\
\hline 64 & 0.0027 & 0.10 & 0.43 & 0.71 & 0.95 & 0.38 & 3.49 & 0.75 \\
\hline 64.2 & 0.0029 & 0.10 & 0.44 & 0.75 & 0.94 & 0.33 & 3.20 & 0.80 \\
\hline 64.4 & 0.0033 & 0.10 & 0.46 & 0.79 & 0.93 & 0.31 & 2.84 & 0.85 \\
\hline 64.6 & 0.0035 & 0.11 & 0.51 & 0.80 & 1.01 & 0.30 & 2.88 & 0.79 \\
\hline 64.8 & 0.0034 & 0.08 & 0.47 & 0.79 & 0.98 & 0.24 & 2.88 & 0.80 \\
\hline 65.1 & 0.0037 & 0.09 & 0.58 & 0.93 & 1.12 & 0.25 & 3.05 & 0.83 \\
\hline 65.3 & 0.0039 & 0.11 & 0.62 & 1.02 & 1.23 & 0.29 & 3.20 & 0.83 \\
\hline 65.5 & 0.0036 & 0.10 & 0.58 & 0.96 & 1.19 & 0.29 & 3.32 & 0.80 \\
\hline 65.7 & 0.0038 & 0.14 & 0.71 & 1.20 & 1.48 & 0.36 & 3.88 & 0.81 \\
\hline 65.6 & 0.0027 & 0.15 & 0.66 & 1.09 & 1.39 & 0.55 & 5.21 & 0.79 \\
\hline 65.6 & 0.0027 & 0.15 & 0.66 & 1.09 & 1.39 & 0.55 & 5.21 & 0.79 \\
\hline 65.8 & 0.0027 & 0.15 & 0.71 & 1.19 & 1.44 & 0.57 & 5.40 & 0.83 \\
\hline 66 & 0.0034 & 0.17 & 0.77 & 1.30 & 1.59 & 0.51 & 4.70 & 0.82 \\
\hline 66.22 & 0.0036 & 0.24 & 1.05 & 1.81 & 2.18 & 0.67 & 5.99 & 0.83 \\
\hline 66.42 & 0.0037 & 0.25 & 1.08 & 1.91 & 2.42 & 0.67 & 6.48 & 0.79 \\
\hline 66.62 & 0.0041 & 0.40 & 1.50 & 2.73 & 3.35 & 0.99 & 8.19 & 0.81 \\
\hline 66.82 & 0.0055 & 0.55 & 3.42 & 6.97 & 9.18 & 0.99 & 16.69 & 0.76 \\
\hline 67.02 & 0.0060 & 0.46 & 1.72 & 3.49 & 4.88 & 0.77 & 8.15 & 0.72 \\
\hline 67.22 & 0.0064 & 0.45 & 1.73 & 3.24 & 4.52 & 0.71 & 7.12 & 0.72 \\
\hline 67.42 & 0.0062 & 0.33 & 1.47 & 2.72 & 3.90 & 0.54 & 6.34 & 0.70 \\
\hline 67.72 & 0.0064 & 0.26 & 1.33 & 2.32 & 3.47 & 0.40 & 5.42 & 0.67 \\
\hline 67.92 & 0.0056 & 0.26 & 1.29 & 2.21 & 3.27 & 0.47 & 5.88 & 0.68 \\
\hline 68.12 & 0.0050 & 0.20 & 1.17 & 2.03 & 3.07 & 0.41 & 6.15 & 0.66 \\
\hline 68.32 & 0.0042 & 0.16 & 1.04 & 1.80 & 2.69 & 0.37 & 6.39 & 0.67 \\
\hline 68.52 & 0.0037 & 0.14 & 0.94 & 1.61 & 2.31 & 0.37 & 6.25 & 0.70 \\
\hline 68.72 & 0.0036 & 0.12 & 1.01 & 1.73 & 2.63 & 0.34 & 7.40 & 0.66 \\
\hline 68.92 & 0.0043 & 0.14 & 1.05 & 1.75 & 2.67 & 0.33 & 6.26 & 0.66 \\
\hline 69.22 & 0.0042 & 0.13 & 0.97 & 1.67 & 2.62 & 0.32 & 6.31 & 0.63 \\
\hline 69.42 & 0.0035 & 0.13 & 0.93 & 1.57 & 2.46 & 0.38 & 7.09 & 0.64 \\
\hline 69.62 & 0.0038 & 0.16 & 1.00 & 1.70 & 2.65 & 0.41 & 6.96 & 0.64 \\
\hline 69.82 & 0.0042 & 0.16 & 0.92 & 1.64 & 2.54 & 0.38 & 6.06 & 0.65 \\
\hline 70.02 & 0.0034 & 0.15 & 0.88 & 1.52 & 2.32 & 0.44 & 6.77 & 0.66 \\
\hline 70.22 & 0.0041 & 0.16 & 1.17 & 1.92 & 2.86 & 0.38 & 7.02 & 0.67 \\
\hline 70.42 & 0.0047 & 0.16 & 1.22 & 2.07 & 2.98 & 0.35 & 6.32 & 0.70 \\
\hline 70.72 & 0.0041 & 0.15 & 0.88 & 1.52 & 2.43 & 0.36 & 5.91 & 0.62 \\
\hline 70.92 & 0.0052 & 0.17 & 1.38 & 2.20 & 3.23 & 0.34 & 6.23 & 0.68 \\
\hline 71.12 & 0.0051 & 0.16 & 1.42 & 2.27 & 3.18 & 0.32 & 6.28 & 0.71 \\
\hline 71.32 & 0.0049 & 0.17 & 1.34 & 2.18 & 3.23 & 0.34 & 6.52 & 0.68 \\
\hline 71.52 & 0.0050 & 0.16 & 1.28 & 2.12 & 2.96 & 0.32 & 5.87 & 0.72 \\
\hline 71.72 & 0.0055 & 0.18 & 1.46 & 2.34 & 3.43 & 0.33 & 6.24 & 0.68 \\
\hline 71.92 & 0.0055 & 0.19 & 1.49 & 2.36 & 3.30 & 0.34 & 5.96 & 0.72 \\
\hline 72.22 & 0.0052 & 0.18 & 1.39 & 2.21 & 3.14 & 0.34 & 6.05 & 0.70 \\
\hline 72.42 & 0.0055 & 0.16 & 1.19 & 1.92 & 2.88 & 0.28 & 5.20 & 0.67 \\
\hline 72.62 & 0.0040 & 0.15 & 0.85 & 1.45 & 2.26 & 0.38 & 5.71 & 0.64 \\
\hline 72.82 & 0.0043 & 0.15 & 0.81 & 1.39 & 2.00 & 0.34 & 4.65 & 0.69 \\
\hline 73.02 & 0.0044 & 0.16 & 0.86 & 1.43 & 2.01 & 0.37 & 4.59 & 0.71 \\
\hline 73.22 & 0.0042 & 0.14 & 0.78 & 1.33 & 1.77 & 0.33 & 4.21 & 0.76 \\
\hline
\end{tabular}


Table 1 (continued).

\begin{tabular}{|c|c|c|c|c|c|c|c|c|}
\hline $\begin{array}{l}\text { Depth } \\
\text { (mbsf) }\end{array}$ & $\begin{array}{c}X \\
\left(\mu \mathrm{m}^{3} \mathrm{~kg}^{-1}\right)\end{array}$ & $\begin{array}{c}\text { ARM } \\
\left(\mathrm{mAm}^{3} \mathrm{~kg}^{-1}\right)\end{array}$ & $\begin{array}{c}\text { IRM } \\
\left(40 \mathrm{mT}^{3}\right) \\
\left(\mathrm{mAm}^{3} \mathrm{~kg}^{-1}\right)\end{array}$ & $\begin{array}{c}\text { IRM } \\
(100 \mathrm{mT}) \\
\left(\mathrm{mAm}^{3} \mathrm{~kg}^{-1}\right)\end{array}$ & $\begin{array}{c}\text { SIRM } \\
\left(\mathrm{mAm}^{3} \mathrm{~kg}^{-1}\right)\end{array}$ & $\begin{array}{c}\mathrm{ARM} / X \\
\left(\mathrm{~K} \mathrm{Am}^{-1}\right)\end{array}$ & $\begin{array}{l}\mathrm{SIRM} / X \\
\left(\mathrm{~K} \mathrm{Am}^{-1}\right)\end{array}$ & $S$ ratio \\
\hline 73.42 & 0.0044 & 0.14 & 0.84 & 1.40 & 1.80 & 0.31 & 4.12 & 0.77 \\
\hline 73.72 & 0.0040 & 0.16 & 0.81 & 1.38 & 1.81 & 0.40 & 4.51 & 0.77 \\
\hline 73.92 & 0.0043 & 0.18 & 0.89 & 1.50 & 1.91 & 0.43 & 4.49 & 0.79 \\
\hline 74.12 & 0.0042 & 0.19 & 0.83 & 1.40 & 1.80 & 0.46 & 4.34 & 0.78 \\
\hline 74.32 & 0.0047 & 0.20 & 0.91 & 1.58 & 1.90 & 0.42 & 4.07 & 0.83 \\
\hline 74.52 & 0.0046 & 0.25 & 1.01 & 1.78 & 2.23 & 0.55 & 4.90 & 0.80 \\
\hline 74.72 & 0.0046 & 0.27 & 1.08 & 1.88 & 2.37 & 0.58 & 5.14 & 0.80 \\
\hline 74.92 & 0.0053 & 0.55 & 0.98 & 1.66 & 2.01 & 1.04 & 3.82 & 0.83 \\
\hline 75.1 & 0.0048 & 0.40 & 1.40 & 2.60 & 3.26 & 0.84 & 6.86 & 0.80 \\
\hline 75.3 & 0.0056 & 0.31 & 1.30 & 2.37 & 2.82 & 0.56 & 5.08 & 0.84 \\
\hline 75.45 & 0.0059 & 0.31 & 1.43 & 2.74 & 3.56 & 0.52 & 6.06 & 0.77 \\
\hline 75.65 & 0.0067 & 0.29 & 1.38 & 2.41 & 3.46 & 0.43 & 5.18 & 0.70 \\
\hline 75.85 & 0.0056 & 0.30 & 1.50 & 2.47 & 3.56 & 0.54 & 6.38 & 0.69 \\
\hline 76.05 & 0.0063 & 0.34 & 1.64 & 2.81 & 4.13 & 0.54 & 6.51 & 0.68 \\
\hline 76.25 & 0.0071 & 0.79 & 2.00 & 6.37 & 8.08 & 1.11 & 11.39 & 0.79 \\
\hline 76.45 & 0.0058 & 0.42 & 2.40 & 7.14 & 8.89 & 0.74 & 15.47 & 0.80 \\
\hline 76.65 & 0.0060 & 0.34 & 1.53 & 2.56 & 3.66 & 0.57 & 6.08 & 0.70 \\
\hline 76.95 & 0.0058 & 0.35 & 1.52 & 2.59 & 3.64 & 0.60 & 6.25 & 0.71 \\
\hline 77.15 & 0.0059 & 0.34 & 1.44 & 2.48 & 3.57 & 0.58 & 6.07 & 0.69 \\
\hline 77.35 & 0.0056 & 0.36 & 1.48 & 2.51 & 3.73 & 0.65 & 6.68 & 0.67 \\
\hline 77.55 & 0.0051 & 0.28 & 1.16 & 2.01 & 2.81 & 0.55 & 5.54 & 0.71 \\
\hline 77.75 & 0.0049 & 0.30 & 1.31 & 2.14 & 2.92 & 0.62 & 5.94 & 0.73 \\
\hline 77.95 & 0.0051 & 0.28 & 1.21 & 2.11 & 2.82 & 0.55 & 5.57 & 0.75 \\
\hline 78.15 & 0.0045 & 0.19 & 0.90 & 1.49 & 1.95 & 0.42 & 4.38 & 0.77 \\
\hline 78.45 & 0.0046 & 0.19 & 0.98 & 1.68 & 2.16 & 0.42 & 4.71 & 0.78 \\
\hline 78.65 & 0.0047 & 0.20 & 0.96 & 1.58 & 2.08 & 0.43 & 4.42 & 0.76 \\
\hline 78.85 & 0.0046 & 0.18 & 0.95 & 1.69 & 2.17 & 0.38 & 4.73 & 0.78 \\
\hline 79.05 & 0.0051 & 0.19 & 0.93 & 1.59 & 2.08 & 0.38 & 4.08 & 0.76 \\
\hline 79.25 & 0.0045 & 0.15 & 0.84 & 1.41 & 1.80 & 0.33 & 3.99 & 0.78 \\
\hline 79.45 & 0.0049 & 0.15 & 0.79 & 1.29 & 1.65 & 0.31 & 3.39 & 0.78 \\
\hline 79.65 & 0.0048 & 0.15 & 0.75 & 1.24 & 1.61 & 0.31 & 3.38 & 0.77 \\
\hline 79.95 & 0.0026 & 0.08 & 0.40 & 0.56 & 0.81 & 0.30 & 3.06 & 0.69 \\
\hline 80.15 & 0.0026 & 0.11 & 0.44 & 0.70 & 0.84 & 0.43 & 3.24 & 0.84 \\
\hline 80.35 & 0.0021 & 0.09 & 0.42 & 0.67 & 0.80 & 0.42 & 3.78 & 0.84 \\
\hline 80.55 & 0.0023 & 0.10 & 0.46 & 0.76 & 0.92 & 0.42 & 3.95 & 0.83 \\
\hline 80.75 & 0.0025 & 0.09 & 0.46 & 0.73 & 0.90 & 0.38 & 3.68 & 0.81 \\
\hline 80.95 & 0.0022 & 0.10 & 0.46 & 0.69 & 0.88 & 0.46 & 4.04 & 0.78 \\
\hline 81.15 & 0.0026 & 0.10 & 0.42 & 0.64 & 0.80 & 0.39 & 3.13 & 0.80 \\
\hline 81.45 & 0.0024 & 0.10 & 0.42 & 0.67 & 0.81 & 0.42 & 3.32 & 0.83 \\
\hline 81.65 & 0.0020 & 0.09 & 0.40 & 0.69 & 0.87 & 0.43 & 4.30 & 0.79 \\
\hline 81.85 & 0.0023 & 0.10 & 0.42 & 0.69 & 0.84 & 0.44 & 3.65 & 0.82 \\
\hline 82.05 & 0.0023 & 0.10 & 0.44 & 0.69 & 0.89 & 0.44 & 3.90 & 0.78 \\
\hline 82.25 & 0.0027 & 0.10 & 0.47 & 0.72 & 0.88 & 0.38 & 3.21 & 0.83 \\
\hline 82.45 & 0.0027 & 0.10 & 0.46 & 0.73 & 0.89 & 0.37 & 3.35 & 0.82 \\
\hline 82.65 & 0.0026 & 0.10 & 0.44 & 0.69 & 0.86 & 0.36 & 3.23 & 0.81 \\
\hline 83.95 & 0.0028 & 0.10 & 0.46 & 0.82 & 0.88 & 0.37 & 3.18 & 0.93 \\
\hline 83.15 & 0.0027 & 0.08 & 0.44 & 0.68 & 0.89 & 0.32 & 3.36 & 0.76 \\
\hline 83.35 & 0.0023 & 0.08 & 0.43 & 0.67 & 0.84 & 0.33 & 3.57 & 0.810 \\
\hline 83.55 & 0.0027 & 0.09 & 0.48 & 0.84 & 0.99 & 0.34 & 3.68 & 0.84 \\
\hline 83.75 & 0.0026 & 0.11 & 0.50 & 0.79 & 0.97 & 0.42 & 3.67 & 0.81 \\
\hline 83.95 & 0.0022 & 0.10 & 0.46 & 0.77 & 1.00 & 0.48 & 4.63 & 0.77 \\
\hline 84.15 & 0.0020 & 0.10 & 0.43 & 0.69 & 0.83 & 0.50 & 4.12 & 0.83 \\
\hline 84.45 & 0.0024 & 0.10 & 0.43 & 0.74 & 0.86 & 0.42 & 3.59 & 0.86 \\
\hline 84.65 & 0.0024 & 0.09 & 0.43 & 0.75 & 0.96 & 0.39 & 4.04 & 0.78 \\
\hline 84.85 & 0.0028 & 0.11 & 0.46 & 0.75 & 0.89 & 0.39 & 3.19 & 0.84 \\
\hline 85.05 & 0.0025 & 0.10 & 0.45 & 0.72 & 0.86 & 0.39 & 3.44 & 0.84 \\
\hline 84.6 & 0.0021 & 0.10 & 0.45 & 0.77 & 0.94 & 0.46 & 4.54 & 0.82 \\
\hline 84.8 & 0.0029 & 0.09 & 0.56 & 0.92 & 1.12 & 0.30 & 3.85 & 0.82 \\
\hline 85 & 0.0030 & 0.10 & 0.74 & 1.14 & 1.32 & 0.34 & 4.41 & 0.86 \\
\hline 85.2 & 0.0029 & 0.11 & 0.60 & 0.97 & 1.21 & 0.37 & 4.24 & 0.80 \\
\hline 85.4 & 0.0028 & 0.11 & 0.58 & 1.08 & 1.24 & 0.41 & 4.45 & 0.87 \\
\hline 85.6 & 0.0026 & 0.12 & 0.62 & 1.00 & 1.22 & 0.44 & 4.67 & 0.82 \\
\hline 85.8 & 0.0026 & 0.12 & 0.57 & 0.96 & 1.18 & 0.46 & 4.54 & 0.81 \\
\hline 86 & 0.0027 & 0.13 & 0.59 & 1.01 & 1.22 & 0.47 & 4.48 & 0.82 \\
\hline 86.2 & 0.0030 & 0.12 & 0.58 & 0.94 & 1.14 & 0.40 & 3.81 & 0.82 \\
\hline 86.5 & 0.0032 & 0.12 & 0.55 & 0.88 & 1.11 & 0.37 & 3.52 & 0.79 \\
\hline 86.7 & 0.0032 & 0.15 & 0.74 & 1.19 & 1.40 & 0.48 & 4.43 & 0.84 \\
\hline 86.9 & 0.0030 & 0.13 & 0.74 & 1.22 & 1.48 & 0.42 & 4.87 & 0.82 \\
\hline 87.1 & 0.0034 & 0.12 & 0.71 & 1.16 & 1.42 & 0.36 & 4.17 & 0.82 \\
\hline 87.3 & 0.0033 & 0.14 & 0.73 & 1.28 & 1.48 & 0.42 & 4.50 & 0.87 \\
\hline 87.5 & 0.0031 & 0.14 & 0.71 & 1.15 & 1.41 & 0.44 & 4.47 & 0.82 \\
\hline 87.7 & 0.0033 & 0.13 & 0.72 & 1.15 & 1.42 & 0.39 & 4.31 & 0.81 \\
\hline 88 & 0.0037 & 0.15 & 0.84 & 1.39 & 1.64 & 0.41 & 4.38 & 0.85 \\
\hline 88.2 & 0.0039 & 0.15 & 0.77 & 1.23 & 1.48 & 0.39 & 3.77 & 0.83 \\
\hline 88.4 & 0.0041 & 0.16 & 0.62 & 1.36 & 1.69 & 0.40 & 4.15 & 0.81 \\
\hline 88.6 & 0.0044 & 0.13 & 0.75 & 1.24 & 1.53 & 0.30 & 3.50 & 0.81 \\
\hline 88.8 & 0.0046 & 0.17 & 0.93 & 1.50 & 1.84 & 0.37 & 3.96 & 0.82 \\
\hline 89 & 0.0050 & 0.14 & 0.86 & 1.41 & 1.76 & 0.29 & 3.55 & 0.80 \\
\hline 89.2 & 0.0055 & 0.17 & 0.97 & 1.54 & 1.90 & 0.30 & 3.48 & 0.81 \\
\hline 89.5 & 0.0045 & 0.17 & 1.00 & 1.67 & 2.03 & 0.38 & 4.48 & 0.82 \\
\hline 89.7 & 0.0056 & 0.16 & 0.97 & 1.57 & 1.90 & 0.28 & 3.38 & 0.83 \\
\hline 89.9 & 0.0000 & 0.19 & 1.03 & 1.63 & 2.02 & & & 0.81 \\
\hline 90.1 & 0.0059 & 0.17 & 0.92 & 1.48 & 1.73 & 0.28 & 2.91 & 0.85 \\
\hline 90.3 & 0.0051 & 0.15 & 0.73 & 1.19 & 1.47 & 0.29 & 2.87 & 0.81 \\
\hline
\end{tabular}


Table 1 (continued).

\begin{tabular}{|c|c|c|c|c|c|c|c|c|}
\hline $\begin{array}{l}\text { Depth } \\
\text { (mbsf) }\end{array}$ & $\begin{array}{c}X \\
\left(\mu \mathrm{m}^{3} \mathrm{~kg}^{-1}\right)\end{array}$ & $\begin{array}{c}\text { ARM } \\
\left(\mathrm{mAm}^{3} \mathrm{~kg}^{-1}\right)\end{array}$ & $\begin{array}{c}\text { IRM } \\
(40 \mathrm{mT}) \\
\left(\mathrm{mAm}^{3} \mathrm{~kg}^{-1}\right)\end{array}$ & $\begin{array}{c}\text { IRM } \\
(100 \mathrm{mT}) \\
\left(\mathrm{mAm}^{3} \mathrm{~kg}^{-1}\right)\end{array}$ & $\begin{array}{c}\text { SIRM } \\
\left(\mathrm{mAm}^{3} \mathrm{~kg}^{-1}\right)\end{array}$ & $\begin{array}{c}\mathrm{ARM} / X \\
\left(\mathrm{~K} \mathrm{Am}^{-1}\right)\end{array}$ & $\begin{array}{l}\mathrm{SIRM} / X \\
\left(\mathrm{~K} \mathrm{Am}^{-1}\right)\end{array}$ & $S$ ratio \\
\hline 90.5 & 0.0053 & 0.15 & 0.90 & 1.41 & 1.68 & 0.29 & 3.18 & 0.84 \\
\hline 91 & 0.0049 & 0.13 & 0.71 & 1.13 & 1.37 & 0.27 & 2.79 & 0.82 \\
\hline 91.2 & 0.0042 & 0.13 & 0.66 & 1.04 & 1.26 & 0.31 & 3.00 & 0.83 \\
\hline 91.4 & 0.0043 & 0.12 & 0.65 & 1.06 & 1.29 & 0.28 & 3.02 & 0.83 \\
\hline 91.6 & 0.0045 & 0.10 & 0.59 & 0.92 & 1.14 & 0.22 & 2.56 & 0.81 \\
\hline 91.8 & 0.0033 & 0.09 & 1.03 & 0.80 & 0.99 & 0.27 & 3.02 & 0.81 \\
\hline 92 & 0.0046 & 0.12 & 0.58 & 0.94 & 1.16 & 0.27 & 2.53 & 0.80 \\
\hline 92.2 & 0.0046 & 0.10 & 0.60 & 0.96 & 1.17 & 0.22 & 2.57 & 0.82 \\
\hline 92.5 & 0.0045 & 0.13 & 0.62 & 0.99 & 1.19 & 0.28 & 2.67 & 0.83 \\
\hline 92.7 & 0.0044 & 0.15 & 0.69 & 1.09 & 1.32 & 0.33 & 2.98 & 0.83 \\
\hline 92.9 & 0.0048 & 0.12 & 0.62 & 0.97 & 1.15 & 0.24 & 2.41 & 0.84 \\
\hline 93.1 & 0.0042 & 0.12 & 0.62 & 0.97 & 1.18 & 0.28 & 2.78 & 0.82 \\
\hline 93.3 & 0.0044 & 0.12 & 0.63 & 0.98 & 1.21 & 0.28 & 2.77 & 0.81 \\
\hline 93.5 & 0.0048 & 0.14 & 0.61 & 0.95 & 1.16 & 0.28 & 2.38 & 0.82 \\
\hline 93.7 & 0.0048 & 0.11 & 0.64 & 0.98 & 1.17 & 0.22 & 2.46 & 0.84 \\
\hline 94 & 0.0047 & 0.10 & 0.60 & 0.91 & 1.15 & 0.21 & 2.47 & 0.79 \\
\hline 94.2 & 0.0045 & 0.11 & 0.64 & 1.01 & 1.22 & 0.25 & 2.70 & 0.83 \\
\hline 94.4 & 0.0048 & 0.12 & 0.61 & 0.96 & 1.18 & 0.25 & 2.45 & 0.81 \\
\hline 94.6 & 0.0044 & 0.12 & 0.55 & 0.90 & 1.16 & 0.27 & 2.63 & 0.77 \\
\hline 94.1 & 0.0049 & 0.09 & 0.60 & 1.01 & 1.17 & 0.18 & 2.38 & 0.86 \\
\hline 94.42 & 0.0051 & 0.13 & 0.61 & 0.76 & 1.24 & 0.26 & 2.42 & 0.61 \\
\hline 94.62 & 0.0041 & 0.11 & 0.50 & 0.78 & 0.96 & 0.27 & 2.36 & 0.81 \\
\hline 94.82 & 0.0041 & 0.13 & 0.54 & 0.84 & 1.05 & 0.32 & 2.57 & 0.80 \\
\hline 95.02 & 0.0043 & 0.11 & 0.47 & 0.78 & 0.98 & 0.26 & 2.27 & 0.80 \\
\hline 95.22 & 0.0041 & 0.12 & 0.54 & 0.90 & 1.11 & 0.30 & 2.70 & 0.81 \\
\hline 95.42 & 0.0036 & 0.11 & 0.47 & 0.79 & 0.93 & 0.30 & 2.60 & 0.85 \\
\hline 95.62 & 0.0037 & 0.07 & 0.46 & 0.78 & 0.93 & 0.19 & 2.51 & 0.83 \\
\hline 95.92 & 0.0038 & 0.11 & 0.46 & 0.77 & 0.94 & 0.29 & 2.47 & 0.82 \\
\hline 96.12 & 0.0037 & 0.06 & 0.44 & 0.79 & 0.91 & 0.16 & 2.50 & 0.86 \\
\hline 96.32 & 0.0037 & 0.11 & 0.45 & 0.74 & 0.86 & 0.29 & 2.31 & 0.87 \\
\hline 96.52 & 0.0035 & 0.11 & 0.43 & 0.71 & 0.81 & 0.31 & 2.35 & 0.88 \\
\hline 96.72 & 0.0034 & 0.13 & 0.50 & 0.81 & 1.00 & 0.38 & 2.98 & 0.81 \\
\hline 96.92 & 0.0037 & 0.06 & 0.44 & 0.73 & 0.92 & 0.16 & 2.48 & 0.79 \\
\hline 97.12 & 0.0036 & 0.12 & 0.50 & 0.81 & 1.02 & 0.33 & 2.81 & 0.79 \\
\hline 97.42 & 0.0042 & 0.12 & 0.47 & 0.80 & 1.00 & 0.29 & 2.37 & 0.81 \\
\hline 97.62 & 0.0036 & 0.10 & 0.44 & 0.72 & 0.87 & 0.29 & 2.41 & 0.83 \\
\hline 97.82 & 0.0035 & 0.11 & 0.43 & 0.71 & 0.87 & 0.32 & 2.49 & 0.82 \\
\hline 98.02 & 0.0037 & 0.08 & 0.46 & 0.77 & 0.97 & 0.21 & 2.64 & 0.79 \\
\hline 98.22 & 0.0034 & 0.08 & 0.43 & 0.74 & 0.92 & 0.22 & 2.70 & 0.80 \\
\hline 98.42 & 0.0030 & 0.06 & 0.41 & 0.69 & 0.88 & 0.21 & 2.89 & 0.79 \\
\hline 98.62 & 0.0030 & 0.12 & 0.43 & 0.73 & 0.92 & 0.40 & 3.07 & 0.79 \\
\hline 98.92 & 0.0028 & 0.06 & 0.36 & 0.73 & 0.91 & 0.23 & 3.27 & 0.80 \\
\hline 99.12 & 0.0034 & 0.07 & 0.45 & 0.75 & 0.89 & 0.20 & 2.66 & 0.84 \\
\hline 99.32 & 0.0029 & 0.12 & 0.44 & 0.71 & 0.89 & 0.41 & 3.13 & 0.80 \\
\hline 99.52 & 0.0029 & 0.06 & 0.39 & 0.68 & 0.87 & 0.21 & 2.99 & 0.79 \\
\hline 99.72 & 0.0033 & 0.10 & 0.34 & 0.71 & 0.87 & 0.32 & 2.67 & 0.82 \\
\hline 99.92 & 0.0027 & 0.11 & 0.39 & 0.71 & 0.84 & 0.39 & 3.08 & 0.84 \\
\hline 100.12 & 0.0023 & 0.07 & 0.42 & 0.76 & 0.93 & 0.29 & 3.99 & 0.81 \\
\hline 100.42 & 0.0027 & 0.06 & 0.40 & 0.67 & 0.85 & 0.23 & 3.14 & 0.78 \\
\hline 100.62 & 0.0032 & 0.10 & 0.38 & 0.67 & 0.83 & 0.31 & 2.62 & 0.81 \\
\hline 100.82 & 0.0028 & 0.12 & 0.42 & 0.72 & 0.87 & 0.42 & 3.14 & 0.82 \\
\hline 101.02 & 0.0025 & 0.09 & 0.38 & 0.63 & 0.81 & 0.38 & 3.29 & 0.78 \\
\hline 101.22 & 0.0028 & 0.10 & 0.40 & 0.68 & 0.89 & 0.36 & 3.13 & 0.77 \\
\hline 101.42 & 0.0028 & 0.09 & 0.38 & 0.67 & 0.84 & 0.34 & 3.03 & 0.80 \\
\hline 101.62 & 0.0026 & 0.11 & 0.37 & 0.65 & 0.75 & 0.41 & 2.90 & 0.86 \\
\hline 101.92 & 0.0026 & 0.10 & 0.42 & 0.70 & 0.87 & 0.39 & 3.31 & 0.80 \\
\hline 102.12 & 0.0026 & 0.11 & 0.42 & 0.76 & 0.94 & 0.40 & 3.58 & 0.81 \\
\hline 102.32 & 0.0025 & 0.05 & 0.39 & 0.65 & 0.83 & 0.21 & 3.36 & 0.79 \\
\hline 102.52 & 0.0023 & 0.11 & 0.42 & 0.70 & 0.92 & 0.47 & 3.97 & 0.76 \\
\hline 102.72 & 0.0027 & 0.08 & 0.37 & 0.80 & 0.98 & 0.28 & 3.62 & 0.81 \\
\hline 102.92 & 0.0025 & 0.07 & 0.43 & 0.75 & 0.94 & 0.28 & 3.76 & 0.80 \\
\hline 103.12 & 0.0024 & 0.10 & 0.37 & 0.67 & 0.83 & 0.40 & 3.47 & 0.80 \\
\hline 103.42 & 0.0022 & 0.08 & 0.34 & 0.74 & 0.89 & 0.37 & 3.98 & 0.84 \\
\hline 103.62 & 0.0025 & 0.12 & 0.45 & 0.77 & 0.95 & 0.48 & 3.73 & 0.81 \\
\hline 103.82 & 0.0025 & 0.10 & 0.45 & 0.80 & 0.96 & 0.42 & 3.86 & 0.83 \\
\hline 104.02 & 0.0022 & 0.07 & 0.43 & 0.75 & 0.93 & 0.30 & 4.26 & 0.81 \\
\hline 103.6 & 0.0024 & 0.12 & 0.41 & 0.76 & 0.92 & 0.48 & 3.76 & 0.83 \\
\hline 103.8 & 0.0030 & 0.15 & 0.58 & 0.99 & 1.27 & 0.51 & 4.20 & 0.79 \\
\hline 104 & 0.0031 & 0.16 & 0.58 & 1.05 & 1.28 & 0.51 & 4.15 & 0.82 \\
\hline 104.2 & 0.0029 & 0.10 & 0.57 & 1.03 & 1.25 & 0.35 & 4.34 & 0.83 \\
\hline 104.4 & 0.0000 & & & & & & & \\
\hline 105.1 & 0.0035 & 0.17 & 0.69 & 1.16 & 1.47 & 0.49 & 4.25 & 0.79 \\
\hline 105.3 & 0.0029 & 0.13 & 0.54 & 0.95 & 1.20 & 0.45 & 4.21 & 0.79 \\
\hline 105.5 & 0.0029 & 0.13 & 0.55 & 0.99 & 1.19 & 0.47 & 4.16 & 0.83 \\
\hline 105.7 & 0.0024 & 0.12 & 0.52 & 0.92 & 1.17 & 0.50 & 4.90 & 0.79 \\
\hline 105.9 & 0.0024 & 0.13 & 0.45 & 0.89 & 1.08 & 0.52 & 4.42 & 0.82 \\
\hline 106.1 & 0.0021 & 0.10 & 0.43 & 0.79 & 0.98 & 0.47 & 4.72 & 0.81 \\
\hline 106.3 & 0.0018 & 0.08 & 0.33 & 0.60 & 0.81 & 0.44 & 4.50 & 0.74 \\
\hline 106.6 & 0.0022 & 0.08 & 0.33 & 0.57 & 0.74 & 0.36 & 3.34 & 0.77 \\
\hline 106.8 & 0.0024 & 0.09 & 0.31 & 0.63 & 0.81 & 0.39 & 3.36 & 0.78 \\
\hline 107 & 0.0019 & 0.08 & 0.29 & 0.58 & 0.72 & 0.43 & 3.73 & 0.80 \\
\hline 107.2 & 0.0013 & 0.03 & 0.32 & 0.59 & 0.74 & 0.64 & 5.58 & 0.80 \\
\hline 107.33 & 0.0017 & 0.07 & 0.26 & 0.49 & 0.59 & 0.44 & 3.44 & 0.84 \\
\hline 107.6 & 0.0022 & 0.10 & 0.38 & 0.66 & 0.77 & 0.43 & 3.47 & 0.86 \\
\hline
\end{tabular}


Table 1 (continued).

\begin{tabular}{|c|c|c|c|c|c|c|c|c|}
\hline $\begin{array}{l}\text { Depth } \\
\text { (mbsf) }\end{array}$ & $\begin{array}{c}X \\
\left(\mu \mathrm{m}^{3} \mathrm{~kg}^{-1}\right)\end{array}$ & $\begin{array}{c}\text { ARM } \\
\left(\mathrm{mAm}^{3} \mathrm{~kg}^{-1}\right)\end{array}$ & $\begin{array}{c}\text { IRM } \\
(40 \mathrm{mT}) \\
\left(\mathrm{mAm}^{3} \mathrm{~kg}^{-1}\right)\end{array}$ & $\begin{array}{c}\text { IRM } \\
(100 \mathrm{mT}) \\
\left(\mathrm{mAm}^{3} \mathrm{~kg}^{-1}\right)\end{array}$ & $\begin{array}{c}\text { SIRM } \\
\left(\mathrm{mAm}^{3} \mathrm{~kg}^{-1}\right)\end{array}$ & $\begin{array}{c}\mathrm{ARM} / X \\
\left(\mathrm{~K} \mathrm{Am}^{-1}\right)\end{array}$ & $\begin{array}{l}\mathrm{SIRM} / X \\
\left(\mathrm{~K} \mathrm{Am}^{-1}\right)\end{array}$ & $S$ ratio \\
\hline 108.1 & 0.0019 & 0.11 & 0.44 & 0.72 & 0.82 & 0.60 & 4.35 & 0.87 \\
\hline 108.3 & 0.0022 & 0.07 & 0.27 & 0.45 & 0.58 & 0.34 & 2.63 & 0.77 \\
\hline 108.5 & 0.0020 & 0.03 & 0.32 & 0.53 & 0.67 & 0.45 & 3.33 & 0.73 \\
\hline 108.7 & 0.0022 & 0.08 & 0.24 & 0.49 & 0.62 & 0.38 & 2.73 & 0.73 \\
\hline 108.9 & 0.0019 & 0.03 & 0.34 & 0.57 & 0.70 & 0.48 & 3.63 & 0.81 \\
\hline 103.1 & 0.0020 & 0.03 & 0.31 & 0.54 & 0.64 & 0.46 & 3.22 & 0.85 \\
\hline 103.3 & 0.0021 & 0.08 & 0.31 & 0.50 & 0.64 & 0.38 & 3.02 & 0.78 \\
\hline 103.6 & 0.0018 & 0.11 & 0.48 & 0.71 & 0.91 & 0.63 & 4.95 & 0.73 \\
\hline 103.8 & 0.0015 & 0.10 & 0.37 & 0.64 & 0.82 & 0.65 & 5.35 & 0.78 \\
\hline 110 & 0.0021 & 0.09 & 0.27 & 0.52 & 0.66 & 0.43 & 3.03 & 0.73 \\
\hline 110.2 & 0.0019 & 0.03 & 0.36 & 0.63 & 0.80 & 0.50 & 4.24 & 0.80 \\
\hline 110.4 & 0.0017 & 0.03 & 0.36 & 0.63 & 0.78 & 0.50 & 4.53 & 0.81 \\
\hline 110.6 & 0.0025 & 0.10 & 0.36 & 0.65 & 0.81 & 0.39 & 3.24 & 0.81 \\
\hline 110.78 & 0.0034 & 0.03 & 0.35 & 0.63 & 0.74 & 0.26 & 2.20 & 0.85 \\
\hline 111.1 & 0.0014 & 0.03 & 0.26 & 0.44 & 0.47 & 0.65 & 3.37 & 0.93 \\
\hline 111.3 & 0.0014 & 0.08 & 0.25 & 0.42 & 0.54 & 0.58 & 3.88 & 0.73 \\
\hline 111.55 & 0.0017 & 0.03 & 0.22 & 0.48 & 0.62 & 0.55 & 3.70 & 0.77 \\
\hline 111.68 & 0.0015 & 0.03 & 0.26 & 0.44 & 0.56 & 0.61 & 3.67 & 0.73 \\
\hline 111.88 & 0.0013 & 0.09 & 0.28 & 0.44 & 0.55 & 0.66 & 4.15 & 0.81 \\
\hline 112.08 & 0.0013 & 0.03 & 0.26 & 0.44 & 0.56 & 0.65 & 4.14 & 0.78 \\
\hline 112.28 & 0.0013 & 0.03 & 0.29 & 0.52 & 0.56 & 0.69 & 4.24 & 0.92 \\
\hline 112.46 & 0.0013 & 0.10 & 0.26 & 0.55 & 0.67 & 0.75 & 5.19 & 0.82 \\
\hline 112.68 & 0.0019 & 0.03 & 0.30 & 0.53 & 0.65 & 0.49 & 3.47 & 0.81 \\
\hline 112.88 & 0.0017 & 0.10 & 0.29 & 0.55 & 0.61 & 0.57 & 3.55 & 0.90 \\
\hline 113.13 & 0.0019 & 0.10 & 0.31 & 0.61 & 0.72 & 0.52 & 3.75 & 0.85 \\
\hline 113.38 & 0.0022 & 0.11 & 0.37 & 0.65 & 0.77 & 0.50 & 3.48 & 0.84 \\
\hline 113.58 & 0.0021 & 0.10 & 0.33 & 0.58 & 0.76 & 0.48 & 3.57 & 0.77 \\
\hline 113.78 & 0.0019 & 0.10 & 0.39 & 0.65 & 0.78 & 0.52 & 4.03 & 0.83 \\
\hline 113.96 & 0.0024 & 0.10 & 0.35 & 0.67 & 0.78 & 0.41 & 3.22 & 0.87 \\
\hline 114.18 & 0.0022 & 0.11 & 0.38 & 0.67 & 0.82 & 0.51 & 3.74 & 0.81 \\
\hline 114.38 & 0.0023 & 0.11 & 0.36 & 0.80 & 0.73 & 0.46 & 3.15 & 1.03 \\
\hline 114.68 & 0.0027 & 0.13 & 0.41 & 0.77 & 0.98 & 0.47 & 3.66 & 0.78 \\
\hline 114.88 & 0.0023 & 0.12 & 0.42 & 0.85 & 1.04 & 0.54 & 4.59 & 0.82 \\
\hline 115.08 & 0.0026 & 0.14 & 0.42 & 0.81 & 0.98 & 0.52 & 3.77 & 0.84 \\
\hline 115.28 & 0.0025 & 0.12 & 0.44 & 0.77 & 0.94 & 0.49 & 3.78 & 0.83 \\
\hline 115.46 & 0.0031 & 0.13 & 0.47 & 0.84 & 0.98 & 0.42 & 3.20 & 0.85 \\
\hline 115.68 & 0.0031 & 0.13 & 0.51 & 0.94 & 1.08 & 0.42 & 3.44 & 0.87 \\
\hline 115.88 & 0.0035 & 0.13 & 0.46 & 0.84 & 1.08 & 0.37 & 3.08 & 0.79 \\
\hline 116.18 & 0.0030 & 0.12 & 0.51 & 0.36 & 1.03 & 0.41 & 3.59 & 0.88 \\
\hline 116.38 & 0.0036 & 0.15 & 0.55 & 0.95 & 1.17 & 0.41 & 3.21 & 0.81 \\
\hline 116.58 & 0.0033 & 0.16 & 0.61 & 1.06 & 1.31 & 0.48 & 3.97 & 0.81 \\
\hline 116.78 & 0.0033 & 0.12 & 0.59 & 1.12 & 1.37 & 0.37 & 4.19 & 0.82 \\
\hline 116.96 & 0.0038 & 0.13 & 0.74 & 1.32 & 1.48 & 0.34 & 3.88 & 0.89 \\
\hline 117.18 & 0.0035 & 0.13 & 0.67 & 1.31 & 1.61 & 0.39 & 4.65 & 0.81 \\
\hline 117.68 & 0.0041 & 0.19 & 0.77 & 1.41 & 1.66 & 0.46 & 4.07 & 0.85 \\
\hline 117.88 & 0.0045 & 0.15 & 0.76 & 1.42 & 1.62 & 0.33 & 3.58 & 0.88 \\
\hline 118.08 & 0.0043 & 0.14 & 0.73 & 1.46 & 1.70 & 0.33 & 3.98 & 0.86 \\
\hline 118.28 & 0.0035 & 0.18 & 0.68 & 1.48 & 1.68 & 0.53 & 4.81 & 0.88 \\
\hline 118.46 & 0.0033 & 0.15 & 0.84 & 1.55 & 1.81 & 0.45 & 5.46 & 0.86 \\
\hline 118.68 & 0.0029 & 0.17 & 0.70 & 1.38 & 1.70 & 0.57 & 5.84 & 0.81 \\
\hline 118.88 & 0.0023 & 0.15 & 0.69 & 1.30 & 1.60 & 0.65 & 6.93 & 0.81 \\
\hline 119.18 & 0.0028 & 0.13 & 0.65 & 1.29 & I. 51 & 0.46 & 5.46 & 0.85 \\
\hline 119.38 & 0.0034 & 0.17 & 0.66 & 1.24 & 1.55 & 0.50 & 4.53 & 0.80 \\
\hline 119.58 & 0.0032 & 0.15 & 0.66 & 1.30 & 1.53 & 0.49 & 4.86 & 0.85 \\
\hline 119.78 & 0.0029 & 0.11 & 0.65 & 1.20 & 1.46 & 0.40 & 5.07 & 0.82 \\
\hline 119.96 & 0.0028 & 0.12 & 0.69 & 1.34 & 1.62 & 0.45 & 5.88 & 0.83 \\
\hline 120.18 & 0.0034 & 0.11 & 0.67 & 1.21 & 1.45 & 0.32 & 4.31 & 0.84 \\
\hline
\end{tabular}

Gartner, this volume) were used to constrain isotopic stage assignment. The lowest occurrence (LO) of Emiliania huxleyi was located at $29.4 \mathrm{mbsf}$ and indicates the position of stages 9 through 8 , while the highest occurrence (HO) of Pseudoemiliania lacunosa at 32.4 mbsf indicates the position of stage 12 . The foraminiferal datum event, the $\mathrm{HO}$ of the pink-pigmented foraminifer Globigerinoides ruber, was used to fix the position of the stage $6 / 5$ e oxygen isotope boundary (Thompson et al., 1979) at about 18 mbsf.

The Brunhes/Matuyama boundary has been identified at $41 \mathrm{mbsf}$, and the top of the Jaramillo Subchron was recorded between 115 and 120 mbsf (Barton et al., this volume); both were used to define the approximate position of the stage $19 / 20$ boundary and stage 28 , respectively.

According to the biostratigraphy the foraminiferal $\delta^{18} \mathrm{O}$ stratigraphy at Hole $819 \mathrm{~A}$ appears to be incomplete. A major hiatus is present at $32.5 \mathrm{mbsf}$, as indicated by the condensed nature of the nannofossil stratigraphy (i.e., the $\mathrm{HO}$ of $P$. lacunosa, $0.465 \mathrm{Ma}$ ) is almost at the same depth as the location of E. huxleyi $(0.275 \mathrm{Ma})$. Also, the upper part of the isotopic record is punctuated with several minor hiatuses. In our interpretation, part of glacial stage 6 and possibly stage 5 is absent. Clearly, no easy solution is available for the problem of isotopic stage assignment above $32.5 \mathrm{mbsf}$ at Hole 819A. 


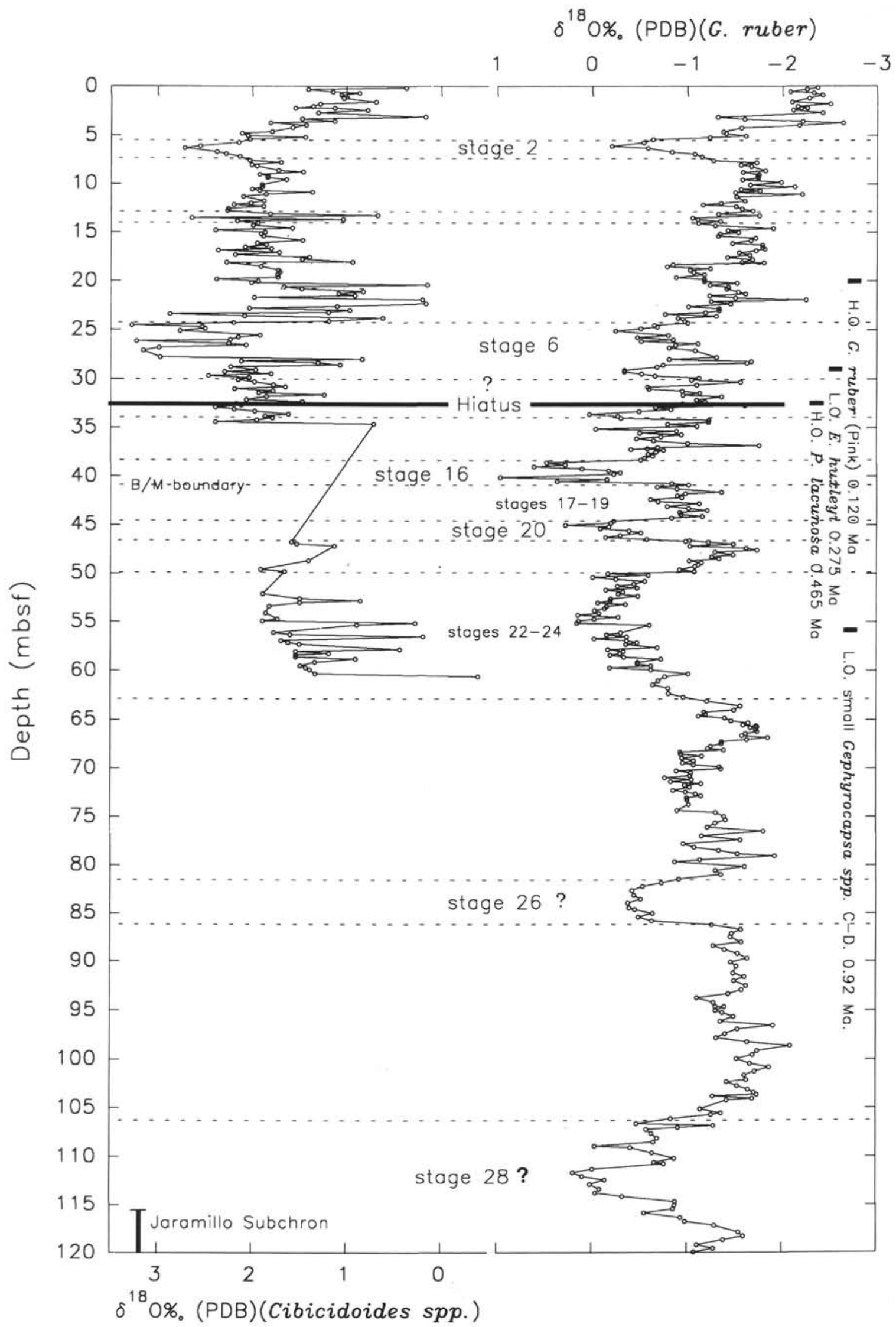

Figure 2. Planktonic (G. ruber) and benthic (Cibicidoides spp.) foraminiferal oxygen isotope records plotted vs. depth at Hole 819A. The solid horizontal line at 32.5 mbsf marks the position of a major stratigraphical hiatus. 
Table 2. Planktonic and benthic foraminiferal oxygen isotope ratios.

\begin{tabular}{|c|c|c|c|c|c|c|c|c|c|c|c|}
\hline Sample & $\begin{array}{l}\text { Depth } \\
\text { (mbst) }\end{array}$ & $\begin{array}{l}\text { G. ruber } \\
\delta^{18} \mathrm{O} \% \\
\text { (PDB) }\end{array}$ & $\begin{array}{c}\text { Cibicidoides spp. } \\
\delta^{18} \mathrm{O} \% \\
\text { (PDB) }\end{array}$ & Sample & $\begin{array}{l}\text { Depth } \\
\text { (mbsf) }\end{array}$ & $\begin{array}{l}\text { G. ruber } \\
8^{18} \mathrm{O} \% \\
\text { (PDB) }\end{array}$ & $\begin{array}{c}\text { Cibicidoides spp. } \\
\delta^{18} \mathrm{O} \% \\
(\mathrm{PDB})\end{array}$ & Sample & $\begin{array}{l}\text { Depth } \\
\text { (mbsf) }\end{array}$ & $\begin{array}{l}\text { G. ruber } \\
\delta^{18} \mathrm{O} \% \\
\text { (PDB) }\end{array}$ & $\begin{array}{c}\text { Cibicidoides spp. } \\
\delta^{18} \mathrm{O} \% \\
\text { (PDB) }\end{array}$ \\
\hline $1 \mathrm{H}-1,16-18$ & 0.16 & -2.407 & 0.332 & $3 \mathrm{H}-1,131-132$ & 19.31 & -1.216 & 1.689 & $5 \mathrm{H}-1,70-72$ & 37.7 & -0.629 & \\
\hline $1 \mathrm{H}-1,30-32$ & 0.3 & -2.295 & 1.368 & $3 \mathrm{H}-2,10-12$ & 19.6 & -0.917 & 1.688 & $5 \mathrm{H}-1,90-92$ & 37.9 & -0.679 & \\
\hline $1 \mathrm{H}-1,54-56$ & 0.54 & -2.117 & 1.106 & $3 \mathrm{H}-2,30-32$ & 19.8 & -1.214 & 2.339 & $5 \mathrm{H}-1,110-112$ & 38.1 & -0.59 & \\
\hline $1 \mathrm{H}-1,70-72$ & 0.7 & -2.366 & 0.827 & $3 \mathrm{H}-2,52-54$ & 20.02 & -1.214 & 1.897 & $5 \mathrm{H}-1,130-132$ & 38.3 & -0.553 & \\
\hline $1 \mathrm{H}-1,90-92$ & 0.9 & -2.46 & 1.018 & $3 \mathrm{H}-2,70-72$ & 20.2 & -1.558 & 1.97 & $5 \mathrm{H}-2,10-12$ & 38.6 & 0.441 & \\
\hline $1 \mathrm{H}-1,120-122$ & 1.2 & -2.315 & 0.995 & $3 \mathrm{H}-2,90-92$ & 20.4 & -1.275 & 0.108 & $5 \mathrm{H}-2,30-32$ & 38.8 & 0.245 & \\
\hline $1 \mathrm{H}-2,10-12$ & 1.6 & -2.133 & 0.652 & $3 \mathrm{H}-2,109-111$ & 20.59 & -1.466 & 1.624 & $5 \mathrm{H}-2,50-52$ & 39 & 0.573 & \\
\hline $1 \mathrm{H}-2,30-32$ & 1.8 & -2.544 & 1.247 & $3 \mathrm{H}-2,130-132$ & 20.81 & -1.45 & 1.435 & $5 \mathrm{H}-2,70-72$ & 39.2 & 0.071 & \\
\hline $1 \mathrm{H}-2,54-56$ & 2.04 & -2.194 & 1.319 & $3 \mathrm{H}-3,10-12$ & 21.1 & -1.574 & 0.784 & $5 \mathrm{H}-2,90-92$ & 39.4 & -0.213 & \\
\hline $1 \mathrm{H}-2,68-70$ & 2.18 & -2.293 & 1.506 & $3 \mathrm{H}-3,30-32$ & 21.3 & -1.648 & 1.856 & $5 \mathrm{H}-2,110-112$ & 39.6 & -0.333 & \\
\hline $1 \mathrm{H}-2,70-72$ & 2.2 & -2.232 & 1.091 & $3 \mathrm{H}-3,52-54$ & 21.52 & -1.269 & 0.87 & $5 \mathrm{H}-2,130-132$ & 39.8 & -0.264 & \\
\hline $1 \mathrm{H}-2,90-92$ & 2.4 & -2.47 & 0.743 & $3 \mathrm{H}-3,70-72$ & 21.7 & -1.542 & 1.94 & $5 \mathrm{H}-3,10-12$ & 40.1 & 0.927 & \\
\hline $1 \mathrm{H}-2,120-122$ & 2.7 & -2.458 & 1.266 & $3 \mathrm{H}-3,90-92$ & 21.9 & -2.285 & 0.16 & $5 \mathrm{H}-3,30-32$ & 40.3 & -0.193 & \\
\hline $1 \mathrm{H}-3,10-12$ & 3.1 & -1.355 & 0.13 & $3 \mathrm{H}-3,109-111$ & 22.09 & -1.286 & & $5 \mathrm{H}-3,50-52$ & 40.5 & 0.329 & \\
\hline $1 \mathrm{H}-3,30-32$ & 3.3 & -1.633 & 1.434 & $3 \mathrm{H}-3,131-132$ & 22.31 & -1.493 & 0.121 & $5 \mathrm{H}-3,70-72$ & 40.7 & -0.876 & \\
\hline $1 \mathrm{H}-3,54-56$ & 3.54 & -2.247 & 1.091 & $3 \mathrm{H}-4,10-12$ & 22.6 & -1.048 & 1.062 & $5 \mathrm{H}-3,90-92$ & 40.9 & -1.052 & \\
\hline $1 \mathrm{H}-3,70-72$ & 3.7 & -2.676 & 1.769 & $3 \mathrm{H}-4,30-32$ & 22.8 & -1.37 & 1.988 & $5 \mathrm{H}-3,110-112$ & 41.1 & -0.725 & \\
\hline $1 \mathrm{H}-3,95-97$ & 3.95 & -2.21 & 1.392 & $3 \mathrm{H}-4,52-54$ & 23.02 & -1.371 & 0.927 & $5 \mathrm{H}-3,130-132$ & 41.3 & -0.93 & \\
\hline $1 \mathrm{H}-3,120-122$ & 4.2 & -1.601 & 1.534 & $3 \mathrm{H}-4,70-72$ & 23.2 & -1.23 & 1.154 & $5 \mathrm{H}-4,10-12$ & 41.6 & -1.398 & \\
\hline $1 \mathrm{H}-4,10-12$ & 4.6 & -1.412 & 1.752 & $3 \mathrm{H}-4,90-92$ & 23.4 & -0.805 & 2.835 & $5 \mathrm{H}-4,30-32$ & 41.8 & -1.016 & \\
\hline $1 \mathrm{H}-4,30-32$ & 4.8 & -1.442 & 2.073 & $3 \mathrm{H}-4,109-111$ & 23.59 & -1.34 & 2.042 & $5 \mathrm{H}-4,50-52$ & 42 & -0.932 & \\
\hline $1 \mathrm{H}-4,54-56$ & 5.04 & -1.649 & 2.011 & $3 \mathrm{H}-4,131-133$ & 23.81 & -0.936 & 0.576 & $5 \mathrm{H}-4,70-72$ & 42.2 & -0.979 & \\
\hline $1 \mathrm{H}-4,70-72$ & 5.2 & -1.269 & 1.403 & $3 \mathrm{H}-5,10-12$ & 24.1 & -1.009 & 3.158 & $5 \mathrm{H}-4,90-92$ & 42.4 & -0.653 & \\
\hline $1 \mathrm{H}-4,90-92$ & 5.4 & -0.675 & 1.988 & $3 \mathrm{H}-5,30-32$ & 24.3 & -1.037 & 2.156 & $5 \mathrm{H}-4,110-112$ & 42.6 & -0.731 & \\
\hline $1 \mathrm{H}-4,120-122$ & 5.7 & -0.573 & 2.107 & $3 \mathrm{H}-5,52-54$ & 24.52 & -0.69 & 3.238 & $5 \mathrm{H}-4,130-132$ & 42.8 & -1.165 & \\
\hline $1 \mathrm{H}-5,10-12$ & 6.1 & -0.24 & 2.514 & $3 \mathrm{H}-5,70-72$ & 24.7 & -0.719 & 2.49 & $5 \mathrm{H}-5,10-12$ & 43.1 & -0.829 & \\
\hline 1H $-5,30-32$ & 6.3 & -0.621 & 2.678 & $3 \mathrm{H}-5,87-89$ & 24.87 & -0.546 & 2.461 & $5 \mathrm{H}-5,30-32$ & 43.3 & -1.052 & \\
\hline $1 \mathrm{H}-5,70-72$ & 6.7 & -0.872 & 2.337 & $3 \mathrm{H}-5,109-111$ & 25.09 & -0.281 & 2.723 & $5 \mathrm{H}-5,50-52$ & 43.5 & -1.247 & \\
\hline $1 \mathrm{H}-5,90-92$ & 6.9 & -1.109 & 2.242 & $3 \mathrm{H}-6,7-9$ & 25.57 & -0.837 & 1.878 & $5 \mathrm{H}-5,70-72$ & 43.7 & -0.959 & \\
\hline $1 \mathrm{H}-5,120-122$ & 7.2 & -1.186 & 2.095 & $3 \mathrm{H}-6,26-28$ & 25.76 & -0.51 & 2.109 & $5 \mathrm{H}-5,90-92$ & 43.9 & -0.971 & \\
\hline $1 \mathrm{H}-6,10-12$ & 7.6 & -1.306 & 1.991 & $3 \mathrm{H}-6,52-54$ & 26.02 & -0.887 & & $5 \mathrm{H}-5,110-112$ & 44.1 & -1.197 & \\
\hline $1 \mathrm{H}-6,30-32$ & 7.8 & -1.761 & 1.658 & $3 \mathrm{H}-6,67-69$ & 26.17 & -0.551 & 3.188 & $5 \mathrm{H}-5,130-132$ & 44.3 & -0.876 & \\
\hline $1 \mathrm{H}-6,54-56$ & 8.04 & -1.599 & 1.968 & $3 \mathrm{H}-6,90-92$ & 26.4 & -1.147 & 2.206 & $5 \mathrm{H}-6,10-12$ & 44.6 & -0.267 & \\
\hline $1 \mathrm{H}-6,68-70$ & 8.18 & -1.708 & 1.914 & $3 \mathrm{H}-6,109-111$ & 26.59 & -0.913 & 2.023 & $5 \mathrm{H}-6,30-32$ & 44.8 & -0.235 & \\
\hline $2 \mathrm{H}-1,12-14$ & 8.62 & -1.853 & 1.683 & $3 \mathrm{H}-6,131-133$ & 26.81 & -0.845 & 2.948 & $5 \mathrm{H}-6,50-52$ & 45 & 0.244 & \\
\hline $2 \mathrm{H}-1,30-32$ & 8.8 & -1.617 & 1.421 & $3 \mathrm{H}-7,10-12$ & 27.1 & -1.118 & 3.113 & $5 \mathrm{H}-6,70-72$ & 45.2 & -0.217 & \\
\hline $2 \mathrm{H}-1,54-56$ & 9.04 & -1.787 & 1.883 & $4 \mathrm{H}-1,30-32$ & 27.8 & -1.343 & 2.934 & $5 \mathrm{H}-6,90-92$ & 45.4 & -0.126 & \\
\hline $2 \mathrm{H}-1,70-72$ & 9.2 & -1.772 & 1.797 & $4 \mathrm{H}-1,50-52$ & 28 & -0.848 & 0.793 & $5 \mathrm{H}-6,110-112$ & 45.6 & -0.426 & \\
\hline $2 \mathrm{H}-1,88-90$ & 9.38 & -1.776 & 1.8 & $4 \mathrm{H}-1,70-72$ & 28.2 & -1.71 & 2.074 & $5 \mathrm{H}-6,130-132$ & 45.8 & -0.55 & \\
\hline $2 \mathrm{H}-1,109-111$ & 9.59 & -1.613 & 1.597 & $4 \mathrm{H}-1,90-92$ & 28.4 & -1.663 & 1.261 & $5 \mathrm{H}-7,10-12$ & 46.1 & -0.334 & \\
\hline $2 \mathrm{H}-1,132-134$ & 9.82 & -2.022 & & $4 \mathrm{H}-1,110-112$ & 28.6 & -0.783 & 1.028 & $5 \mathrm{H}-7,30-32$ & 46.3 & -0.18 & \\
\hline $2 \mathrm{H}-2,12-14$ & 10.12 & -1.695 & 1.86 & $4 \mathrm{H}-1,130-132$ & 28.8 & -0.721 & 2.185 & $5 \mathrm{H}-7,50-52$ & 46.5 & -0.614 & \\
\hline $2 \mathrm{H}-2,30-32$ & 10.3 & -2.165 & 1.855 & $4 \mathrm{H}-2,10-12$ & 29.1 & -0.373 & 1.924 & $5 \mathrm{H}-7,70-72$ & 46.7 & -1.024 & \\
\hline $2 \mathrm{H}-2,54-56$ & 10.54 & -1.599 & 1.965 & $4 \mathrm{H}-2,30-32$ & 29.3 & -0.376 & 2.248 & $6 \mathrm{H}-1,10-12$ & 46.6 & -1.062 & \\
\hline $2 \mathrm{H}-2,70-72$ & 10.7 & -1.793 & 1.885 & $4 \mathrm{H}-2,50-52$ & 29.5 & -0.554 & 1.76 & $6 \mathrm{H}-1,30-32$ & 46.8 & -1.262 & 1.579 \\
\hline $2 \mathrm{H}-2,88-90$ & 10.88 & -1.539 & 1.327 & $4 \mathrm{H}-2,70-72$ & 29.7 & -0.697 & 2.423 & $6 \mathrm{H}-1,50-52$ & 47 & -1.524 & 1.524 \\
\hline $2 \mathrm{H}-2,109-111$ & 11.09 & -2.245 & 1.819 & $4 \mathrm{H}-2,90-92$ & 29.9 & -1.16 & 1.991 & $6 \mathrm{H}-1,70-72$ & 47.2 & -1.068 & 1.127 \\
\hline $2 \mathrm{H}-2,132-134$ & 11.32 & -1.55 & 2.057 & $4 \mathrm{H}-2,110-112$ & 30.1 & -1.082 & 2.109 & $6 \mathrm{H}-1,90-92$ & 47.4 & -1.66 & \\
\hline $2 \mathrm{H}-3,3 \mathrm{O}-32$ & 11.8 & -1.639 & 1.84 & $4 \mathrm{H}-2,130-132$ & 30.3 & -1.599 & 1.935 & $6 \mathrm{H}-1,110-112$ & 47.6 & -1.774 & \\
\hline $2 \mathrm{H}-3,54-56$ & 12.04 & -1.386 & 1.978 & $4 \mathrm{H}-3,10-12$ & 30.6 & -1.134 & 1.733 & $6 \mathrm{H}-1,130-132$ & 47.8 & -1.334 & \\
\hline $2 \mathrm{H}-3,70-72$ & 12.2 & -1.198 & 2.157 & $4 \mathrm{H}-3,30-32$ & 30.8 & -0.62 & 1.607 & $6 \mathrm{H}-2,10-12$ & 48.1 & -1.52 & \\
\hline $2 \mathrm{H}-3,88-90$ & 12.38 & -1.545 & 1.842 & $4 \mathrm{H}-3,50-52$ & 31 & -0.638 & 2.147 & $6 \mathrm{H}-2,30-32$ & 48.3 & -1.299 & \\
\hline $2 \mathrm{H}-3,109-111$ & 12.59 & -1.61 & 2.216 & $4 \mathrm{H}-3,70-72$ & 31.2 & -0.984 & 1.738 & $6 \mathrm{H}-2,50-52$ & 48.5 & -1.373 & \\
\hline $2 \mathrm{H}-3,132-134$ & 12.82 & -1.722 & 2.225 & $4 \mathrm{H}-3,90-92$ & 31.4 & -1.17 & 1.888 & $6 \mathrm{H}-2,70-72$ & 48.7 & -1.059 & 1.398 \\
\hline $2 \mathrm{H}-4,12-14$ & 13.12 & -1.362 & 1.771 & $4 \mathrm{H}-3,110-112$ & 31.6 & -0.991 & 1.196 & $6 \mathrm{H}-2,90-92$ & 48.9 & -1.191 & \\
\hline $2 \mathrm{H}-4,30-32$ & 13.3 & -1.792 & 0.634 & $4 \mathrm{H}-3,130-132$ & 31.8 & -1.4 & 1.807 & $6 \mathrm{H}-2,110-112$ & 49.1 & -1.156 & \\
\hline $2 \mathrm{H}-4,54-56$ & 13.54 & -1.089 & 2.602 & $4 \mathrm{H}-4,10-12$ & 32.1 & -1.135 & 2.02 & $6 \mathrm{H}-2,130-132$ & 49.3 & -1.117 & \\
\hline $2 \mathrm{H}-4,70-72$ & 13.7 & -1.145 & 0.996 & $4 \mathrm{H}-4,30-32$ & 32.3 & -1.222 & 1.427 & $6 \mathrm{H}-3,10-12$ & 49.6 & -0.959 & 1.907 \\
\hline $2 \mathrm{H}-4,88-90$ & 13.88 & -1.381 & 2.12 & $4 \mathrm{H}-4,50-52$ & 32.5 & -0.987 & 2.122 & $6 \mathrm{H}-3,30-32$ & 49.8 & -1.115 & 1.652 \\
\hline $2 \mathrm{H}-4,109-111$ & 14.09 & -1.154 & 1.901 & $4 \mathrm{H}-4,70-72$ & 32.7 & -1.642 & 2.075 & $6 \mathrm{H}-3,50-52$ & 50 & -0.206 & \\
\hline $2 \mathrm{H}-4,132-134$ & 14.34 & -1.328 & 1.953 & $4 \mathrm{H}-4,90-92$ & 32.9 & -0.707 & 2.346 & $6 \mathrm{H}-3,70-72$ & 50.2 & -0.63 & \\
\hline $2 \mathrm{H}-5,12-14$ & 14.62 & -1.936 & 1.537 & $4 \mathrm{H}-4,110-112$ & 33.1 & -0.865 & 2.149 & $6 \mathrm{H}-3,90-92$ & 50.4 & -0.048 & \\
\hline $2 \mathrm{H}-5,30-32$ & 14.8 & -1.461 & 2.351 & $4 \mathrm{H}-4,130-132$ & 33.3 & -0.531 & 1.931 & $6 \mathrm{H}-3,110-112$ & 50.6 & -0.291 & \\
\hline $2 \mathrm{H}-5,54-56$ & 15.04 & -1.571 & 1.827 & $4 \mathrm{H}-5,10-12$ & 33.6 & -0.008 & 1.574 & $6 \mathrm{H}-3,130-132$ & 50.8 & -0.595 & \\
\hline $2 \mathrm{H}-5,70-72$ & 15.2 & -1.38 & 1.868 & $4 \mathrm{H}-5,30-32$ & 33.8 & -0.308 & 1.821 & $6 \mathrm{H}-4,10-12$ & 51.1 & -0.479 & \\
\hline $2 \mathrm{H}-5,88-90$ & 15.38 & -1.36 & 1.841 & $4 \mathrm{H}-5,50-52$ & 34 & -0.342 & 1.74 & $6 \mathrm{H}-4,30-32$ & 51.3 & -0.307 & \\
\hline $2 \mathrm{H}-5,109-111$ & 15.59 & -1.754 & & $4 \mathrm{H}-5,70-72$ & 34.2 & -1.264 & 1.911 & $6 \mathrm{H}-4,50-52$ & 51.5 & -0.514 & \\
\hline $2 \mathrm{H}-5,132-134$ & 15.82 & -1.704 & 1.425 & $4 \mathrm{H}-5,90-92$ & 34.4 & -1.263 & 2.345 & $6 \mathrm{H}-4,70-72$ & 51.7 & -0.185 & \\
\hline $2 \mathrm{H}-6,12-14$ & 16.12 & -1.511 & 1.908 & $4 \mathrm{H}-5,110-112$ & 34.6 & -0.835 & 0.67 & $6 \mathrm{H}-4,90-92$ & 51.9 & -0.362 & \\
\hline $2 \mathrm{H}-6,30-32$ & 16.3 & -1.824 & 1.811 & $4 \mathrm{H}-5,130-132$ & 34.8 & -1.139 & & $6 \mathrm{H}-4,110-112$ & 52.1 & -0.317 & 1.883 \\
\hline $2 \mathrm{H}-6,54-56$ & 16.54 & -1.826 & 2.035 & $4 \mathrm{H}-6,10-12$ & 35.1 & -0.076 & & $6 \mathrm{H}-4,130-132$ & 52.3 & -0.523 & \\
\hline $2 \mathrm{H}-6,70-72$ & 16.7 & -1.847 & 1.759 & $4 \mathrm{H}-6,30-32$ & 35.3 & -0.925 & & $6 \mathrm{H}-5,10-12$ & 52.6 & -0.235 & 1.49 \\
\hline $2 \mathrm{H}-6,88-90$ & 16.88 & -1.755 & 2.321 & $4 \mathrm{H}-6,50-52$ & 35.5 & -0.541 & & $6 \mathrm{H}-5,30-32$ & 52.8 & -0.232 & 0.849 \\
\hline $2 \mathrm{H}-6,109-111$ & 17.09 & -1.579 & 1.675 & $4 \mathrm{H}-6,70-72$ & 35.7 & -0.977 & & $6 \mathrm{H}-5,50-52$ & 53 & -0.1 & 1.49 \\
\hline $2 \mathrm{H}-6,132-134$ & 17.32 & -1.69 & 2.142 & $4 \mathrm{H}-6,90-92$ & 35.9 & -0.76 & & $6 \mathrm{H}-5,70-72$ & 53.2 & -0.39 & \\
\hline $2 \mathrm{H}-7,7-8$ & 17.57 & -1.458 & 1.354 & $4 \mathrm{H}-6,110-112$ & 36.1 & -0.506 & & $6 \mathrm{H}-5,90-92$ & 53.4 & -0.192 & 1.816 \\
\hline $2 \mathrm{H}-7,27-29$ & 17.77 & -1.719 & 1.431 & $4 \mathrm{H}-6,130-132$ & 36.3 & -0.686 & & $6 \mathrm{H}-5,110-112$ & 53.6 & -0.174 & \\
\hline $2 \mathrm{H}-7,54-56$ & 18.04 & -1.611 & 0.897 & $4 \mathrm{H}-7,10-12$ & 36.6 & -1.041 & & $6 \mathrm{H}-5,130-132$ & 53.8 & -0.067 & \\
\hline $3 \mathrm{H}-1,10-12$ & 18.1 & -1.839 & 2.233 & $4 \mathrm{H}-7,30-32$ & 36.8 & -1.795 & & $6 \mathrm{H}-6,10-12$ & 54.1 & -0.114 & 1.852 \\
\hline $3 \mathrm{H}-1,30-32$ & 18.3 & -0.886 & & $4 \mathrm{H}-7,50-52$ & 37 & -0.728 & & $6 \mathrm{H}-6,30-32$ & 54.3 & 0.108 & \\
\hline $3 \mathrm{H}-1,52-54$ & 18.52 & -0.824 & 1.868 & $4 \mathrm{H}-7,70-72$ & 37.2 & -0.448 & & $6 \mathrm{H}-6,50-52$ & 54.5 & -0.314 & \\
\hline $3 \mathrm{H}-1,70-72$ & 18.7 & -1.278 & & $5 \mathrm{H}-1,10-12$ & 37.1 & -0.62 & & $6 \mathrm{H}-6,70-72$ & 54.7 & -0.062 & 1.725 \\
\hline $3 \mathrm{H}-1,90-92$ & 18.9 & -1.065 & 1.683 & $5 \mathrm{H}-1,30-32$ & 37.3 & -0.79 & & $6 \mathrm{H}-6,90-92$ & 54.9 & 0.102 & 1.885 \\
\hline $3 \mathrm{H}-1,109-111$ & 19.09 & -1.101 & 1.666 & $5 \mathrm{H}-1,50-52$ & 37.5 & -0.713 & & $6 \mathrm{H}-6,110-112$ & 55.1 & 0.123 & 0.269 \\
\hline
\end{tabular}


Table 2 (continued).

\begin{tabular}{|c|c|c|c|c|c|c|c|c|c|c|c|}
\hline Sample & $\begin{array}{l}\text { Depth } \\
\text { (mbsf) }\end{array}$ & $\begin{array}{l}\text { G. ruber } \\
\delta^{18} \mathrm{O} \% \\
\text { (PDB) }\end{array}$ & $\begin{array}{c}\text { Cibicidoides spp. } \\
\delta^{18} \mathrm{O} \% \\
\text { (PDB) }\end{array}$ & Sample & $\begin{array}{l}\text { Depth } \\
\text { (mbsf) }\end{array}$ & $\begin{array}{l}\text { G. ruber } \\
\delta^{18} \mathrm{O} \% \\
\text { (PDB) }\end{array}$ & $\begin{array}{c}\text { Cibicidoides spp. } \\
\delta^{18} \mathrm{O} \% \\
\text { (PDB) }\end{array}$ & Sample & $\begin{array}{l}\text { Depth } \\
\text { (mbsf) }\end{array}$ & $\begin{array}{l}\text { G. ruber } \\
\delta^{18} \mathrm{O} \% \\
\text { (PDB) }\end{array}$ & $\begin{array}{c}\text { Cibicidoides spp. } \\
\delta^{18} \mathrm{O} \% \\
(\mathrm{PDB})\end{array}$ \\
\hline $6 \mathrm{H}-6,130-132$ & 55.3 & -0.644 & 0.886 & $8 \mathrm{H}-7,10-12$ & 73.72 & -1.021 & & $10 \mathrm{H}-6,10-12$ & 91 & & \\
\hline $7 \mathrm{H}-1,10-12$ & 56.1 & -0.341 & $\begin{array}{l}1.768 \\
1.768\end{array}$ & $8 \mathrm{H}-7,30-32$ & 73.92 & -1.021 & & $10 \mathrm{H}-6,30-32$ & 91.2 & -1.492 & \\
\hline $7 \mathrm{H}-1,30-32$ & 56.3 & -0.196 & 1.591 & $8 \mathrm{H}-7,50-52$ & 74.12 & & & $10 \mathrm{H}-6,50-52$ & 91.4 & & \\
\hline $7 \mathrm{H}-1,50-52$ & 56.5 & -0.406 & 0.188 & $8 \mathrm{H}-7,70-72$ & 74.32 & -0.897 & & $10 \mathrm{H}-6,70-72$ & 91.6 & -1.609 & \\
\hline $7 \mathrm{H}-1,70-72$ & 56.7 & -0.063 & & $8 \mathrm{H}-7,90-92$ & 74.52 & -1.307 & & $10 \mathrm{H}-6,90-92$ & 91.8 & & \\
\hline $7 \mathrm{H}-1,90-92$ & 56.9 & -0.403 & 1.69 & $8 \mathrm{H}-7,110-112$ & 74.72 & & & $10 \mathrm{H}-6,110-112$ & 92 & -1.5 & \\
\hline $7 \mathrm{H}-1,110-112$ & 57.1 & -0.511 & 1.614 & $8 \mathrm{H}-7,130-132$ & 74.92 & -1.393 & & $10 \mathrm{H}-6,130-132$ & 92.2 & & \\
\hline $7 \mathrm{H}-1,130-132$ & 57.3 & -0.395 & 1.495 & $9 \mathrm{H}-1,10-12$ & 75.1 & & & $10 \mathrm{H}-7,10-12$ & 92.5 & -1.622 & \\
\hline $7 \mathrm{H}-2,10-12$ & 57.6 & -0.727 & & $9 \mathrm{H}-1,30-32$ & 75.3 & -1.412 & & $10 \mathrm{H}-7,30-32$ & 92.7 & & \\
\hline $7 \mathrm{H}-2,30-32$ & 57.8 & -0.207 & 0.433 & $9 \mathrm{H}-2,10-12$ & 75.45 & & & $10 \mathrm{H}-7,50-52$ & 92.9 & -1.578 & \\
\hline $7 \mathrm{H}-2,50-52$ & 58 & -0.369 & 1.531 & $9 \mathrm{H}-2,30-32$ & 75.65 & -1.301 & & $10 \mathrm{H}-7,70-72$ & 93.1 & & \\
\hline $7 \mathrm{H}-2,70-72$ & 58.2 & -0.334 & 1.185 & $9 \mathrm{H}-2,50-52$ & 75.85 & & & $10 \mathrm{H}-7,90-92$ & 93.3 & -1.439 & \\
\hline $7 \mathrm{H}-2,90-92$ & 58.4 & -0.233 & 1.534 & $9 \mathrm{H}-2,70-72$ & 76.05 & -1.217 & & $10 \mathrm{H}-7,110-112$ & 93.5 & & \\
\hline $7 \mathrm{H}-2,110-112$ & 58.6 & -0.374 & 1.535 & $9 \mathrm{H}-2,90-92$ & 76.25 & -1.217 & & $10 \mathrm{H}-7,130-132$ & 93.7 & -1.107 & \\
\hline $7 \mathrm{H}-2,130-132$ & 58.8 & -0.765 & 0.897 & $9 \mathrm{H}-2,110-112$ & 76.45 & -1.806 & & $10 \mathrm{H}-8,10-12$ & 94 & 1.101 & \\
\hline $7 \mathrm{H}-3,10-12$ & 59.1 & -0.516 & 1.333 & $9 \mathrm{H}-2,130-132$ & 76.65 & & & $10 \mathrm{H}-8,30-32$ & 94.2 & -1.286 & \\
\hline $7 \mathrm{H}-3,30-32$ & 59.3 & -0.519 & & $9 \mathrm{H}-3,10-12$ & 76.95 & -1.156 & & $10 \mathrm{H}-8,50-52$ & 94.4 & & \\
\hline $7 \mathrm{H}-3,50-52$ & 59.5 & 1.487 & & $9 \mathrm{H}-3,30-32$ & 77.15 & -1.150 & & $10 \mathrm{H}-8,70-72$ & 94.6 & -1.304 & \\
\hline $7 \mathrm{H}-3,70-72$ & 59.7 & -0.663 & 1.43 & $9 \mathrm{H}-3,50-52$ & 77.35 & -1.56 & & $11 \mathrm{H}-1,10-12$ & 94.1 & 1.507 & \\
\hline $7 \mathrm{H}-3,90-92$ & 59.9 & -0.228 & 1.387 & $9 \mathrm{H}-3,70-72$ & 77.55 & & & $11 \mathrm{H}-2,10-12$ & 94.42 & & \\
\hline $7 \mathrm{H}-3,110-112$ & 60.1 & -0.659 & 1.328 & $9 \mathrm{H}-3,90-92$ & 77.75 & -0.962 & & $11 \mathrm{H}-2,30-32$ & 94.62 & -1.398 & \\
\hline $7 \mathrm{H}-3,130-132$ & 60.3 & -1.01 & -0.394 & $9 \mathrm{H}-3,110-112$ & 77.95 & & & $11 \mathrm{H}-2,50-52$ & 94.82 & & \\
\hline $7 \mathrm{H}-4,10-12$ & 60.6 & -0.768 & & $9 \mathrm{H}-3,130-132$ & 78.15 & -1.075 & & $11 \mathrm{H}-2,70-72$ & 95.02 & -1.303 & \\
\hline $7 \mathrm{H}-4,30-32$ & 60.8 & & & $9 \mathrm{H}-4,10-12$ & 78.45 & -1.333 & & $11 \mathrm{H}-2,90-92$ & 95.22 & -1.375 & \\
\hline $7 \mathrm{H}-4,50-52$ & 61 & -0.696 & & $9 \mathrm{H}-4,30-32$ & 78.65 & & & $11 \mathrm{H}-2,110-112$ & 95.42 & & \\
\hline $7 \mathrm{H}-4,70-72$ & 61.2 & & & $9 \mathrm{H}-4,50-52$ & 78.85 & -1.533 & & $11 \mathrm{H}-2,130-132$ & 95.62 & -1.495 & \\
\hline $7 \mathrm{H}-4,90-92$ & 61.4 & -0.64 & & $9 \mathrm{H}-4,70-72$ & 79.05 & -1.921 & & $11 \mathrm{H}-3,10-12$ & 95.92 & & \\
\hline $7 \mathrm{H}-4,110-112$ & 61.6 & & & $9 \mathrm{H}-4,90-92$ & 79.25 & 1.721 & & $11 \mathrm{H}-3,30-32$ & 96.12 & -1.357 & \\
\hline $7 \mathrm{H}-4,130-132$ & 61.8 & -0.801 & & $9 \mathrm{H}-4,110-112$ & 79.45 & -1.136 & & $11 \mathrm{H}-3,50-52$ & 96.32 & & \\
\hline $7 \mathrm{H}-5,10-12$ & 62.1 & & & $9 \mathrm{H}-4,130-132$ & 79.65 & -0.872 & & $11 \mathrm{H}-3,70-72$ & 96.52 & -1.91 & \\
\hline $7 \mathrm{H}-5,30-32$ & 62.3 & -0.804 & & $9 \mathrm{H}-5,10-12$ & 79.95 & & & $11 \mathrm{H}-3,90-92$ & 96.72 & & \\
\hline $7 \mathrm{H}-5,50-52$ & 62.5 & & & $9 \mathrm{H}-5,30-32$ & 80.15 & -1.608 & & $11 \mathrm{H}-3,110-112$ & 96.92 & -1.536 & \\
\hline $7 \mathrm{H}-5,70-72$ & 62.7 & -0.962 & & $9 \mathrm{H}-5,50-52$ & 80.35 & & & $11 \mathrm{H}-3,130-132$ & 97.12 & & \\
\hline $7 \mathrm{H}-5,90-92$ & 62.9 & & & $9 \mathrm{H}-5,70-72$ & 80.55 & -1.302 & & $11 \mathrm{H}-4,10-12$ & 97.42 & -1.407 & \\
\hline $7 \mathrm{H}-5,110-112$ & 63.1 & -1.208 & & $9 \mathrm{H}-5,90-92$ & 80.75 & & & $11 \mathrm{H}-4,30-32$ & 97.62 & & \\
\hline $7 \mathrm{H}-6,10-12$ & 63.6 & -1.561 & & $9 \mathrm{H}-5,110-112$ & 80.95 & -1.362 & & $11 \mathrm{H}-4,50-52$ & 97.82 & -1.314 & \\
\hline $7 \mathrm{H}-6,30-32$ & 63.8 & & & $9 \mathrm{H}-5,130-132$ & 81.15 & & & $11 \mathrm{H}-4,70-72$ & 98.02 & & \\
\hline $7 \mathrm{H}-6,50-52$ & 64 & -1.495 & & $9 \mathrm{H}-6,10-12$ & 81.45 & -0.916 & & $11 \mathrm{H}-4,90-92$ & 98.22 & -1.639 & \\
\hline $7 \mathrm{H}-6,70-72$ & 64.2 & -1.179 & & $9 \mathrm{H}-6,30-32$ & 81.65 & & & $11 \mathrm{H}-4,110-112$ & 98.42 & & \\
\hline $7 \mathrm{H}-6,90-92$ & 64.4 & -1.197 & & $9 \mathrm{H}-6,50-52$ & 81.85 & -0.734 & & $11 \mathrm{H}-4,130-132$ & 98.62 & -2.091 & \\
\hline $7 \mathrm{H}-6,110-112$ & 64.6 & -1.122 & & $9 \mathrm{H}-6,70-72$ & 82.05 & & & $11 \mathrm{H}-5,10-12$ & 98.92 & & \\
\hline $7 \mathrm{H}-6,130-132$ & 64.8 & -1.397 & & $9 \mathrm{H}-6,90-92$ & 82.25 & -0.535 & & $11 \mathrm{H}-5,30-32$ & 99.12 & -1.744 & \\
\hline $7 \mathrm{H}-7,10-12$ & 65.1 & -1.467 & & $9 \mathrm{H}-6,110-112$ & 82.45 & & & $11 \mathrm{H}-5,50-52$ & 99.32 & & \\
\hline $7 \mathrm{H}-7,30-32$ & 65.3 & -1.647 & & $9 \mathrm{H}-6,130-132$ & 82.65 & -0.423 & & $11 \mathrm{H}-5,70-72$ & 99.52 & -1.695 & \\
\hline $7 \mathrm{H}-7,50-52$ & 65.5 & -1.592 & & $9 \mathrm{H}-7,10-12$ & 83.95 & & & $11 \mathrm{H}-5,90-92$ & 99.72 & & \\
\hline $7 \mathrm{H}-7,70-72$ & 65.7 & -1.737 & & $9 \mathrm{H}-7,30-32$ & 83.15 & -0.444 & & $11 \mathrm{H}-5,110-112$ & 99.92 & -1.527 & \\
\hline $8 \mathrm{H}-1,10-12$ & 65.6 & -1.728 & & $9 \mathrm{H}-7,50-52$ & 83.35 & & & $11 \mathrm{H}-5,130-132$ & 100.12 & & \\
\hline $8 \mathrm{H}-1,30-32$ & 65.8 & -1.667 & & $9 \mathrm{H}-7,70-72$ & 83.55 & -0.514 & & $11 \mathrm{H}-6,10-12$ & 100.42 & -1.666 & \\
\hline $8 \mathrm{H}-1,50-52$ & 66 & -1.723 & & $9 \mathrm{H}-7,90-92$ & 83.75 & & & $11 \mathrm{H}-6,30-32$ & 100.62 & & \\
\hline $8 \mathrm{H}-2,10-12$ & 66.22 & -1.739 & & $9 \mathrm{H}-7,110-112$ & 83.95 & -0.381 & & $11 \mathrm{H}-6,50-52$ & 100.82 & -1.866 & \\
\hline $8 \mathrm{H}-2,30-32$ & 66.42 & -1.615 & & $9 \mathrm{H}-7,130-132$ & 84.15 & & & $11 \mathrm{H}-6,70-72$ & 101.02 & & \\
\hline $8 \mathrm{H}-2,50-52$ & 66.62 & -1.58 & & $9 \mathrm{H}-8,10-12$ & 84.45 & -0.391 & & $11 \mathrm{H}-6,90-92$ & 101.22 & -1.715 & \\
\hline $8 \mathrm{H}-2,70-72$ & 66.82 & -1.851 & & $9 \mathrm{H}-8,30-32$ & 84.65 & & & $11 \mathrm{H}-6,110-112$ & 101.42 & & \\
\hline $8 \mathrm{H}-2,90-92$ & 67.02 & -1.628 & & $9 \mathrm{H}-8,50-52$ & 84.85 & -1.365 & & $11 \mathrm{H}-6,130-132$ & 101.62 & -1.607 & \\
\hline $8 \mathrm{H}-2,110-112$ & 67.22 & -1.366 & & $9 \mathrm{H}-8,70-72$ & 85.05 & & & $11 \mathrm{H}-7,10-12$ & 101.92 & & \\
\hline $8 \mathrm{H}-2,130-132$ & 67.42 & -1.366 & & $10 \mathrm{H}-1,10-12$ & 84.6 & -0.453 & & $11 \mathrm{H}-7,30-32$ & 102.12 & -1.629 & \\
\hline $8 \mathrm{H}-3,10-12$ & 67.72 & -1.249 & & $10 \mathrm{H}-1,30-32$ & 84.8 & & & $11 \mathrm{H}-7,50-52$ & 102.32 & -1.424 & \\
\hline $8 \mathrm{H}-3,30-32$ & 67.92 & -1.222 & & $10 \mathrm{H}-2,10-12$ & 85 & -0.642 & & $11 \mathrm{H}-7,70-72$ & 102.52 & & \\
\hline $8 \mathrm{H}-3,50-52$ & 68.12 & -1.391 & & $10 \mathrm{H}-2,30-32$ & 85.2 & & & $11 \mathrm{H}-7,90-92$ & 102.72 & -1.53 & \\
\hline $8 \mathrm{H}-3,70-72$ & 68.32 & -0.928 & & $10 \mathrm{H}-2,50-52$ & 85.4 & -0.49 & & $11 \mathrm{H}-7,110-112$ & 102.92 & & \\
\hline $8 \mathrm{H}-3,90-92$ & 68.52 & -0.936 & & $10 \mathrm{H}-2,70-72$ & 85.6 & & & $11 \mathrm{H}-7,130-132$ & 103.12 & -1.647 & \\
\hline $8 \mathrm{H}-3,110-112$ & 68.72 & -1.157 & & $10 \mathrm{H}-2,90-92$ & 85.8 & -0.634 & & $11 \mathrm{H}-8,10-12$ & 103.42 & -1.708 & \\
\hline $8 \mathrm{H}-3,130-132$ & 68.92 & -0.947 & & $10 \mathrm{H}-2,110-112$ & 86 & & & $11 \mathrm{H}-8,30-32$ & 103.62 & -1.736 & \\
\hline $8 \mathrm{H}-4,10-12$ & 69.22 & -1.071 & & $10 \mathrm{H}-2,130-132$ & 86.2 & -1.262 & & $11 \mathrm{H}-8,50-52$ & 103.82 & & \\
\hline $8 \mathrm{H}-4,30-32$ & 69.42 & -0.958 & & $10 \mathrm{H}-3,10-12$ & 86.5 & & & $11 \mathrm{H}-8,70-72$ & 104.02 & -1.69 & \\
\hline $8 \mathrm{H}-4,50-52$ & 69.62 & -1.07 & & $10 \mathrm{H}-3,30-32$ & 86.7 & -1.57 & & $12 \mathrm{H}-1,10-12$ & 103.6 & & \\
\hline $8 \mathrm{H}-4,70-72$ & 69.82 & -1.342 & & $10 \mathrm{H}-3,50-52$ & 86.9 & & & $12 \mathrm{H}-1,30-32$ & 103.8 & -1.274 & \\
\hline $8 \mathrm{H}-4,90-92$ & 70.02 & -1.362 & & $10 \mathrm{H}-3,70-72$ & 87.1 & -1.474 & & $12 \mathrm{H}-1,50-52$ & 104 & & \\
\hline $8 \mathrm{H}-4,110-112$ & 70.22 & -0.891 & & $10 \mathrm{H}-3,90-92$ & 87.3 & & & $12 \mathrm{H}-1,70-72$ & 104.2 & -1.421 & \\
\hline $8 \mathrm{H}-4,130-132$ & 70.42 & -1.043 & & $10 \mathrm{H}-3,110-112$ & 87.5 & -1.461 & & $12 \mathrm{H}-1,90-92$ & 104.4 & & \\
\hline $8 \mathrm{H}-5,10-12$ & 70.72 & -1.034 & & $10 \mathrm{H}-3,130-132$ & 87.7 & & & $12 \mathrm{H}-2,10-12$ & 105.1 & -1.147 & \\
\hline $8 \mathrm{H}-5,30-32$ & 70.92 & -0.768 & & $10 \mathrm{H}-4,10-12$ & 88 & -1.575 & & $12 \mathrm{H}-2,30-32$ & 105.3 & & \\
\hline $8 \mathrm{H}-5,50-52$ & 71.12 & -1.048 & & $10 \mathrm{H}-4,30-32$ & 88.2 & & & $12 \mathrm{H}-2,50-52$ & 105.5 & -1.364 & \\
\hline $8 \mathrm{H}-5,70-72$ & 71.32 & -0.83 & & $10 \mathrm{H}-4,50-52$ & 88.4 & -1.282 & & $12 \mathrm{H}-2,70-72$ & 105.7 & -1.259 & \\
\hline $8 \mathrm{H}-5,90-92$ & 71.52 & -1.149 & & $10 \mathrm{H}-4,70-72$ & 88.6 & 88.8 & & $12 \mathrm{H}-2,90-92$ & 105.9 & & \\
\hline $8 \mathrm{H}-5,110-112$ & 71.72 & -0.978 & & $10 \mathrm{H}-4,90-92$ & & & -1.398 & $12 \mathrm{H}-2,110-112$ & 106.1 & -0.834 & \\
\hline $8 \mathrm{H}-5,130-132$ & 71.92 & -1.03 & & $10 \mathrm{H}-4,110-112$ & 89 & & & $12 \mathrm{H}-2,130-132$ & 106.3 & & \\
\hline $8 \mathrm{H}-6,10-12$ & 72.22 & -0.858 & & $10 \mathrm{H}-4,130-132$ & 89.2 & -1.538 & & $12 \mathrm{H}-3,10-12$ & 106.6 & -0.472 & \\
\hline $8 \mathrm{H}-6,30-32$ & 72.42 & -0.986 & & $10 \mathrm{H}-5,10-12$ & 89.5 & & & $12 \mathrm{H}-3,30-32$ & 106.8 & -1.283 & \\
\hline $8 \mathrm{H}-6,50-52$ & 72.62 & -1.09 & & $10 \mathrm{H}-5,30-32$ & 89.7 & -1.638 & & $12 \mathrm{H}-3,50-52$ & 107 & -0.913 & \\
\hline $8 \mathrm{H}-6,70-72$ & 72.82 & -1.149 & & $10 \mathrm{H}-5,50-52$ & 89.9 & & & $12 \mathrm{H}-3,70-72$ & 107.2 & -0.578 & \\
\hline $8 \mathrm{H}-6,90-92$ & 73.02 & -1 & & $10 \mathrm{H}-5,70-72$ & 90.1 & -1.465 & & $12 \mathrm{H}-3,83-85$ & 107.33 & & \\
\hline $8 \mathrm{H}-6,110-112$ & 73.22 & -1.004 & & $10 \mathrm{H}-5,90-92$ & 90.3 & & & $12 \mathrm{H}-3,110-112$ & $107-6$ & -0.634 & \\
\hline $8 \mathrm{H}-6,130-132$ & 73.42 & & & $10 \mathrm{H}-5,110-112$ & 90.5 & -1.523 & & $12 \mathrm{H}-4,10-12$ & 108.1 & -0.691 & \\
\hline
\end{tabular}


Table 2 (continued).

\begin{tabular}{|c|c|c|c|}
\hline Sample & $\begin{array}{l}\text { Depth } \\
\text { (mbsf) }\end{array}$ & $\begin{array}{l}\text { G. ruber } \\
\delta^{18} \mathrm{O} \% \\
(\mathrm{PDB})\end{array}$ & $\begin{array}{c}\text { Cibicidoides spp. } \\
\delta^{18} \mathrm{O} \% \\
\text { (PDB) }\end{array}$ \\
\hline $12 \mathrm{H}-4,30-32$ & 108.3 & & \\
\hline $12 \mathrm{H}-4,50-52$ & 108.5 & -0.654 & \\
\hline $12 \mathrm{H}-4,70-72$ & 108.7 & & \\
\hline $12 \mathrm{H}-4,90-92$ & 108.9 & -0.036 & \\
\hline $12 \mathrm{H}-4,110-112$ & 109.1 & -0.409 & \\
\hline $12 \mathrm{H}-4,130-132$ & 109.3 & -0.767 & \\
\hline $12 \mathrm{H}-5,10-12$ & 109.6 & & \\
\hline $12 \mathrm{H}-5,30-32$ & 109.8 & & \\
\hline $12 \mathrm{H}-5,50-52$ & 110 & & \\
\hline $12 \mathrm{H}-5,70-72$ & 110.2 & -0.874 & \\
\hline $12 \mathrm{H}-5,90-92$ & 110.4 & & \\
\hline $12 \mathrm{H}-5,110-112$ & 110.6 & -0.665 & \\
\hline $12 \mathrm{H}-5,128-130$ & 110.78 & -0.762 & \\
\hline $13 \mathrm{H}-1,10-12$ & 111.1 & & \\
\hline $13 \mathrm{H}-1,30-32$ & 111.3 & -0.013 & \\
\hline $13 \mathrm{H}-1,55-57$ & 111.55 & & \\
\hline $13 \mathrm{H}-2,10-12$ & 111.68 & 0.193 & \\
\hline $13 \mathrm{H}-2,30-32$ & 111.88 & & \\
\hline $13 \mathrm{H}-2,50-52$ & 112.08 & 0.093 & \\
\hline $13 \mathrm{H}-2,70-72$ & 112.28 & & \\
\hline $13 \mathrm{H}-2,88-90$ & 112.46 & -0.144 & \\
\hline $13 \mathrm{H}-2,110-112$ & 112.68 & & \\
\hline $13 \mathrm{H}-2,130-132$ & 112.88 & 0.015 & \\
\hline $13 \mathrm{H}-3,10-12$ & 113.13 & & \\
\hline $13 \mathrm{H}-3,30-32$ & 113.38 & -0.085 & \\
\hline $13 \mathrm{H}-3,50-52$ & 113.58 & & \\
\hline $13 \mathrm{H}-3,70-72$ & 113.78 & -0.045 & \\
\hline $13 \mathrm{H}-3,88-90$ & 113.96 & & \\
\hline $13 \mathrm{H}-3,110-112$ & 114.18 & -0.328 & \\
\hline $13 \mathrm{H}-3,130-132$ & 114.38 & & \\
\hline $13 \mathrm{H}-4,10-12$ & 114.68 & -0.882 & \\
\hline $13 \mathrm{H}-4,30-32$ & 114.88 & & \\
\hline $13 \mathrm{H}-4,50-52$ & 115.08 & -0.875 & \\
\hline $13 \mathrm{H}-4,70-72$ & 115.28 & & \\
\hline $13 \mathrm{H}-4,88-90$ & 115.46 & -0.856 & \\
\hline $13 \mathrm{H}-4,110-112$ & 115.68 & & \\
\hline $13 \mathrm{H}-4,130-132$ & 115.88 & -0.552 & \\
\hline $13 \mathrm{H}-5,10-12$ & 116.18 & & \\
\hline $13 \mathrm{H}-5,30-32$ & 116.38 & -0.935 & \\
\hline $13 \mathrm{H}-5,50-52$ & 116.58 & & \\
\hline $13 \mathrm{H}-5,70-72$ & 116.78 & -0.981 & \\
\hline $13 \mathrm{H}-5,88-90$ & 116.96 & & \\
\hline $13 \mathrm{H}-5,110-112$ & 117.18 & -1.291 & \\
\hline $13 \mathrm{H}-6,10-12$ & 117.68 & & \\
\hline $13 \mathrm{H}-6,30-32$ & 117.88 & -1.543 & \\
\hline $13 \mathrm{H}-6,50-52$ & 118.08 & & \\
\hline $13 \mathrm{H}-6,70-72$ & 118.28 & -1.594 & \\
\hline $13 \mathrm{H}-6,88-90$ & 118.46 & & \\
\hline $13 \mathrm{H}-6,110-112$ & 118.68 & -1.384 & \\
\hline $13 \mathrm{H}-6,130-132$ & 118.88 & & \\
\hline $13 \mathrm{H}-7,10-12$ & 119.18 & -1.107 & \\
\hline $13 \mathrm{H}-7,30-32$ & 119.38 & & \\
\hline $13 \mathrm{H}-7,50-52$ & 119.58 & -1.28 & \\
\hline $13 \mathrm{H}-7,70-72$ & 119.78 & & \\
\hline $13 \mathrm{H}-7,88-90$ & 119.96 & -1.073 & \\
\hline $13 \mathrm{H}-7,110-112$ & 120.18 & & \\
\hline $13 \mathrm{H}-7,130-132$ & 120.38 & -1.138 & \\
\hline
\end{tabular}

Below $32.5 \mathrm{mbsf}$, the isotopic record is thought to be more complete. Isotopic stages 14 through 21 , at about $47 \mathrm{mbsf}$, can be recognized in the record at hole $819 \mathrm{~A}$, except for the absence of a clear stage 18. Below about $50 \mathrm{mbsf}$ continuous correlation with the isotopic record of Raymo et al. (1990) becomes more difficult. For example, some uncertainty exists as to whether the slight enrichment event between 68 and $74 \mathrm{mbsf}$ is in point of fact stage 24 , and not part of stage 25 . Oxygen isotope stage 28 has been placed at about $114 \mathrm{mbsf}$, which is supported by the tentative location of the Jaramillo subchron (0.97-0.91 Ma) between 115-120 mbsf (Barton et al., this volume). This implies that the recorded nannofossil datum level of $\mathrm{HO}$ of small Gephyrocapsa spp. C-D at 56 mbsf (Wei and Gartner, this volume) is unreliable and possibly indicates either reworking or sample contamination.
In general, the benthic foraminiferal oxygen isotope record, relative to the planktonic oxygen isotope record, displays a greater intrastage amplitudinal variation in isotopic value. This greater amplitudinal variation, in isotopic value, is particularly evident within isotope stages 4,7 , and 8 . Benthic oxygen isotope values are typically about 2.5 to $3.5 \%$ heavier than corresponding planktonic oxygen isotope values. The oxygen isotope difference between benthic and planktonic foraminifers $\left(\Delta \delta^{18} \mathrm{O}_{\mathrm{B}-\mathrm{P}}\right)$ are shown in Figure 4. Comparison of the $\Delta \delta^{18} \mathrm{O}_{\mathrm{B}-\mathrm{P}}$ record in the upper and lower parts of the sequence at Hole $819 \mathrm{~A}$ indicates that during stages 22 through 24 the mean isotopic difference is about $1.5 \%$. This compares with about $3.0 \%$ during stage 9 and $3.5 \%$ during the Holocene.

The planktonic foraminiferal oxygen isotope record displays a trend of depletion in mean $\delta^{18} \mathrm{O}$ value, from $32.5 \mathrm{mbsf}$ through the Holocene, of about $1.5 \%$. This upward decreasing trend is not so clear in the benthic foraminiferal curve and is not present below about 42 mbsf in the planktonic curve. Below 42 mbsf, the planktonic foraminiferal curve displays a trend, similar to that displayed by the benthic foraminiferal $\delta^{18} \mathrm{O}$ record, of depletion in mean $\delta^{18} \mathrm{O}$ value, with depth.

\section{Mineral Magnetic Properties}

Figures 5 and 6 summarize the variations of mineral magnetic properties and planktonic foraminiferal oxygen isotope ratio with depth in Hole 819A. Magnetic susceptibility and saturation isothermal magnetization (Fig. 5) predominantly reflect the concentration of magnetite in the sediments. The highest concentrations are found in the top $7 \mathrm{~m}$ and at four horizons at 21.7, 24.6, 67.2, and $76.2 \mathrm{mbsf}$. Other sediment horizons have dramatically reduced magnetite concentrations, thus necessitating the use of logarithmic $X$ and SIRM scales to display the magnetic variations.

Below the marked change in magnetic concentration at $7 \mathrm{mbsf}$ and excluding the peaks at 21.7 and $24.6 \mathrm{mbsf}$, specific magnetic susceptibility and saturation IRM display a general trend of decreasing value with depth to about $32.5 \mathrm{mbsf}$, roughly following the foraminiferal $\delta^{18} \mathrm{O}$ trend (Fig. 5). In general, below $32.5 \mathrm{mbsf}$, high magnetite content occurs during the interglacial periods and low concentrations during the glacial periods (e.g., stages 22 to 24 and stages 26 and 28). By contrast, above 32.5 mbsf the relationship between specific magnetic susceptibility and SIRM is more capricious, with no clear relationship between magnetic concentration and foraminiferal $\delta^{18} \mathrm{O}$ values and with the peaks in magnetic mineral content occurring in both the glacial and interglacial periods.

The mineral magnetic ratios of S-ratio $\left(\mathrm{IRM}_{100 \mathrm{mT}} / \mathrm{IRM}_{1000 \mathrm{mT}}\right)$ and $\mathrm{ARM} / X$ were plotted down the core to $120 \mathrm{mbsf}$ for Hole $819 \mathrm{~A}$ in Figure 6 (Table 1). Both ratios display a marked change in value at about $7.5 \mathrm{mbsf}$, the same depth at which the magnetic concentrations were found to change (Figs. 5 and 7). Both the S-ratio and ARM/X ratio are again high at depths of 21.7 and $24.6 \mathrm{mbsf}$, where the magnetic concentrations also are high. Below $7.5 \mathrm{mbsf}$, both ratios show a slight overall trend of increasing mean value with depth.

Below $32.5 \mathrm{mbsf}$, peaks in magnetic mineral concentration tend to be associated with relatively low percentage carbonate content (see Fig. 8). This inverse relationship is particularly evident in the percentage of carbonate content archive (bulk) sample plot alongside magnetic susceptibility (see Fig. 8). However, subtle variations in this overall antithetical relationship occur, for example, at about 5 and 32 mbsf. Curves for percentages of carbonate and susceptibility exhibit a positive correlation.

\section{Particle Size Characteristics}

The relative proportions of fine fraction $(<63 \mu \mathrm{m})$ and mean particle size $(\mu \mathrm{m})$ with depth at Hole $819 \mathrm{~A}$ are illustrated in Figure 9 


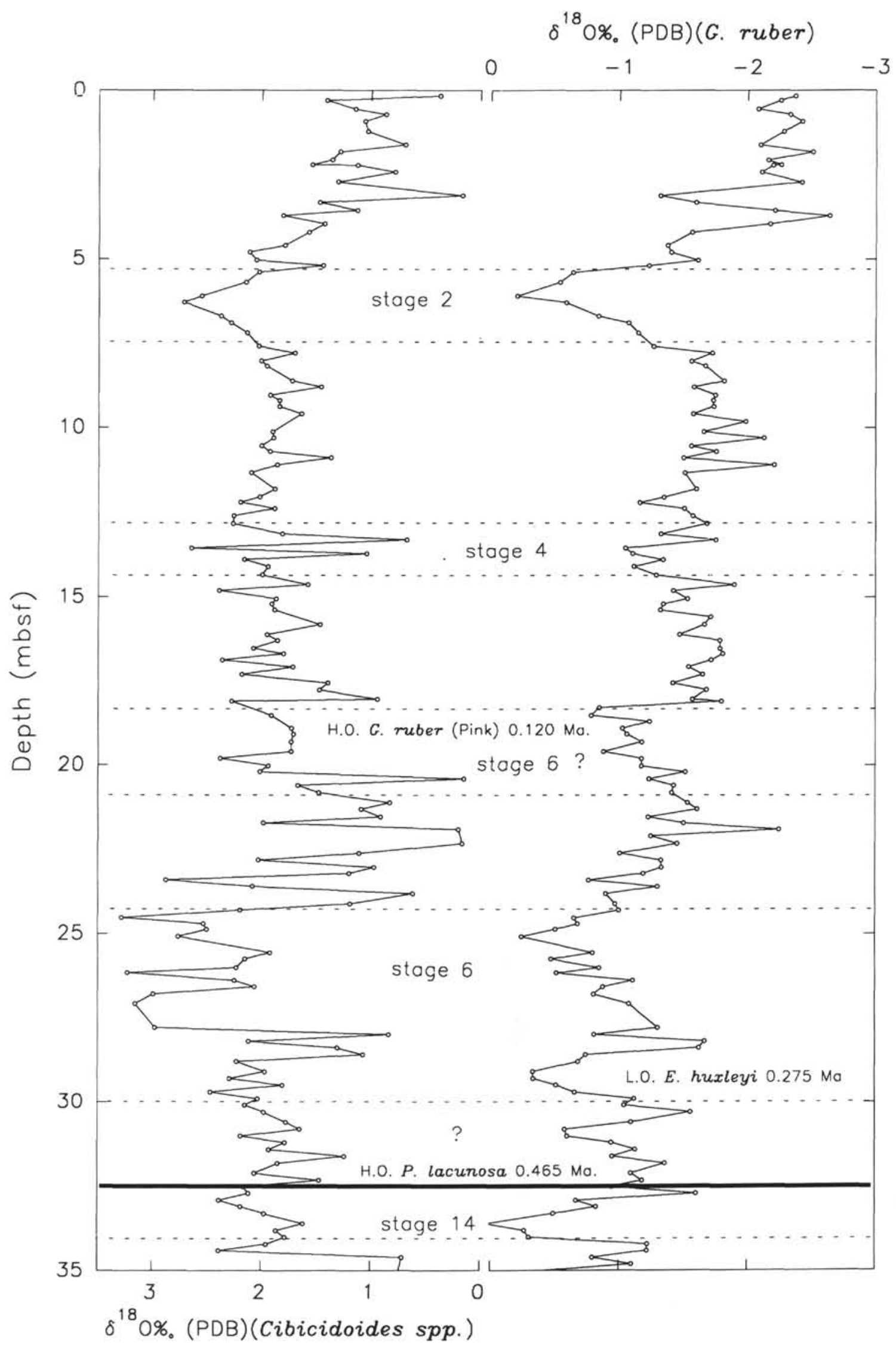

Figure 3. Planktonic (G. ruber) and benthic (Cibicidoides spp.) foraminiferal oxygen isotope records at Hole 819A plotted downcore to 35 mbsf. The solid horizontal line at 32.5 mbsf marks the position of a major stratigraphical hiatus. 
Table 3. Particle size distribution for sampldes collected at Hole 819A.

\begin{tabular}{|c|c|c|c|c|c|c|c|c|c|c|c|}
\hline $\begin{array}{l}\text { Sample } \\
\text { depth } \\
\text { (mbsf) }\end{array}$ & $\begin{array}{c}\text { A } \\
\% \text { fine } \\
\text { fraction } \\
(<63 \mu \mathrm{m})\end{array}$ & $\begin{array}{c}\text { B } \\
\% \text { fine } \\
\text { fraction } \\
(<63 \mu \mathrm{m})\end{array}$ & $\begin{array}{c}\text { Mean } \\
\text { particle size } \\
(\mu \mathrm{m})\end{array}$ & $\begin{array}{l}\text { Sample } \\
\text { depth } \\
\text { (mbsf) }\end{array}$ & $\begin{array}{c}\text { A } \\
\% \text { fine } \\
\text { fraction } \\
(<63 \mu \mathrm{m})\end{array}$ & $\begin{array}{c}\text { B } \\
\% \text { fine } \\
\text { fraction } \\
(<63 \mu \mathrm{m})\end{array}$ & $\begin{array}{c}\text { Mean } \\
\text { particle size } \\
(\mu \mathrm{m})\end{array}$ & $\begin{array}{l}\text { Sample } \\
\text { depth } \\
\text { (mbsf) }\end{array}$ & $\begin{array}{c}\text { A } \\
\% \text { fine } \\
\text { fraction } \\
(<63 \mu \mathrm{m})\end{array}$ & $\begin{array}{c}\text { B } \\
\% \text { fine } \\
\text { fraction } \\
(<63 \mu \mathrm{m})\end{array}$ & $\begin{array}{c}\text { Mean } \\
\text { particle size } \\
(\mu \mathrm{m})\end{array}$ \\
\hline 0.16 & 81.8 & 86.4 & 37.8 & 18.9 & 82.2 & 90.1 & 40.8 & 37.1 & 85.4 & 86.1 & 36.9 \\
\hline 0.3 & 81.6 & 84.4 & 49.0 & 19.09 & 75.2 & 85.3 & 64.1 & 37.3 & 84.5 & 84.5 & 37.0 \\
\hline 0.54 & 81.8 & 90.7 & 47.1 & 19.31 & 78.8 & 88.7 & 54.2 & 37.5 & 82.2 & 86.1 & 39.3 \\
\hline 0.7 & 85.5 & 90.5 & 40.5 & 19.6 & 74.0 & 87.8 & 65.9 & 37.7 & 75.8 & 85.5 & 41.9 \\
\hline 0.9 & 83.3 & 90.6 & 42.1 & 19.8 & 57.5 & 76.6 & 129.3 & 37.9 & 76.9 & 86.2 & 44.3 \\
\hline 1.2 & 88.1 & 93.7 & 36.5 & 20.02 & 72.6 & 83.4 & 77.2 & 38.1 & 79.9 & 84.3 & 41.6 \\
\hline 1.6 & 80.1 & 93.3 & 39.5 & 20.2 & 82.4 & 85.9 & 51.0 & & & & \\
\hline 1.8 & 95.7 & 94.7 & 25.7 & 20.4 & 75.5 & 81.6 & 57.0 & 38.3 & 83.3 & 87.6 & 37.3 \\
\hline 2.04 & 88.4 & 93.1 & 35.7 & 20.59 & 81.0 & 87.8 & 50.2 & 38.6 & 81.3 & 75.5 & 48.7 \\
\hline 2.18 & 92.0 & 93.5 & 32.5 & 20.81 & 80.2 & 87.0 & 46.2 & 38.8 & 84.0 & 81.6 & 36.2 \\
\hline 2.2 & 88.7 & 93.0 & 33.9 & 21.1 & 85.1 & 90.8 & 40.5 & 39 & 83.7 & 81.1 & 41.7 \\
\hline 2.4 & 87.2 & 90.5 & 38.2 & 21.3 & 91.1 & 93.9 & 28.5 & 39.2 & 87.8 & 88.1 & 31.1 \\
\hline 2.7 & 88.7 & 93.8 & 35.9 & 21.52 & 92.3 & 95.3 & 28.4 & 39.4 & 87.3 & 90.6 & 33.9 \\
\hline 3.1 & 83.4 & 92.5 & 40.0 & 21.7 & 90.2 & 95.2 & 31.6 & 39.6 & 76.5 & 80.5 & 47.3 \\
\hline 3.3 & 87.2 & 95.7 & 35.1 & 21.9 & 92.8 & 96.6 & 27.1 & 39.8 & 93.7 & 76.8 & 39.9 \\
\hline 3.54 & 96.9 & 95.6 & 22.8 & 22.09 & 92.8 & 96.7 & 28.0 & 40.1 & 89.5 & 75.4 & 30.6 \\
\hline 3.7 & 91.1 & 96.5 & 29.7 & 22.31 & 99.7 & 97.2 & 17.3 & 40.3 & 86.3 & 85.3 & 35.2 \\
\hline 3.95 & 96.0 & 95.6 & 22.6 & 22.6 & 48.2 & 71.2 & 107.1 & 40.5 & 87.5 & 89.5 & 32.0 \\
\hline 4.2 & 94.2 & 96.0 & 24.9 & 22.8 & 93.0 & 97.5 & 26.4 & 40.7 & 82.4 & 90.2 & 38.6 \\
\hline 4.6 & 92.6 & 95.0 & 25.8 & 23.02 & 97.6 & 98.3 & 18.9 & 40.9 & 85.2 & 87.2 & 36.9 \\
\hline 4.8 & 92.2 & 95.8 & 25.9 & 23.2 & 96.2 & 97.3 & 21.7 & 41.1 & 82.6 & 85.2 & 38.7 \\
\hline 5.04 & 93.0 & 95.0 & 26.6 & 23.4 & 89.7 & 96.1 & 32.5 & 41.3 & 80.8 & 84.9 & 40.8 \\
\hline 5.2 & 85.5 & 93.4 & 38.3 & 23.59 & 98.2 & 98.3 & 19.3 & 41.6 & 82.9 & 87.9 & 38.8 \\
\hline 5.4 & 79.7 & 88.8 & 50.3 & 23.81 & 83.8 & 94.9 & 42.0 & 41.8 & 79.2 & 84.0 & 41.6 \\
\hline 5.7 & 71.7 & 81.0 & 69.3 & 24.1 & 76.9 & 77.5 & 57.9 & 42 & 79.9 & 85.9 & 40.1 \\
\hline 6.1 & 84.1 & 88.7 & 32.7 & 24.3 & 70.6 & 74.1 & 85.5 & 42.2 & 82.8 & 81.4 & 38.0 \\
\hline 6.3 & 64.0 & 70.9 & 89.0 & 24.52 & 63.0 & 78.9 & 128.8 & 42.4 & 81.6 & 84.4 & 40.6 \\
\hline 6.7 & 53.6 & 63.3 & 113.1 & 24.7 & 84.7 & 85.1 & 45.8 & 42.6 & 78.1 & 83.8 & 43.0 \\
\hline 6.9 & 59.3 & 66.3 & 96.9 & 24.87 & 74.8 & 80.6 & 67.8 & 42.8 & 79.0 & 81.7 & 43.2 \\
\hline 7.2 & 58.1 & 70.1 & 97.8 & 25.09 & 73.7 & 67.8 & 80.9 & 43.1 & 72.8 & 79.4 & 48.8 \\
\hline 7.6 & 65.6 & 80.9 & 82.1 & 25.57 & 60.6 & 58.8 & 112.6 & 43.3 & 76.2 & 79.6 & 45.0 \\
\hline 7.8 & 64.9 & 85.0 & 77.5 & 25.76 & 50.1 & 47.6 & 150.5 & 43.5 & 68.3 & 77.7 & 55.3 \\
\hline 8.04 & 78.8 & 85.2 & 52.0 & 26.02 & 59.8 & 63.6 & 120.0 & 43.7 & 77.0 & 81.2 & 42.8 \\
\hline 8.18 & 80.8 & 89.1 & 47.8 & 26.17 & 71.1 & 72.5 & 79.0 & 43.9 & 71.5 & 74.5 & 51.6 \\
\hline 8.62 & 79.5 & 87.6 & 53.6 & 26.4 & 73.6 & 73.3 & 79.6 & 44.1 & 75.1 & 80.0 & 46.9 \\
\hline 8.8 & 84.7 & 90.6 & 43.3 & 26.59 & 55.4 & 52.9 & 148.5 & 44.3 & 77.9 & 81.4 & 43.3 \\
\hline 9.04 & 84.2 & 92.1 & 42.8 & 26.81 & 57.1 & 55.3 & 135.9 & 44.6 & 76.4 & 79.8 & 45.2 \\
\hline 9.2 & 85.4 & 92.6 & 41.1 & 27.1 & 53.0 & 56.3 & 147.4 & 44.8 & 79.0 & 84.2 & 42.8 \\
\hline 9.38 & 90.1 & 93.6 & 35.7 & 27.8 & 79.4 & 85.4 & 59.0 & 45 & 81.3 & 85.6 & 38.8 \\
\hline 9.59 & 87.4 & 93.5 & 31.9 & 28 & 83.0 & 78.8 & 49.8 & 45.2 & 84.9 & 85.4 & 34.1 \\
\hline 9.82 & 89.4 & 92.6 & 32.1 & 28.2 & 100.0 & 95.5 & 16.6 & 45.4 & 81.7 & 85.8 & 39.0 \\
\hline 10.12 & 86.2 & & 38.6 & 28.4 & 100.0 & 95.3 & 16.0 & 45.6 & 84.0 & 88.4 & 35.9 \\
\hline 10.3 & 89.7 & 94.2 & 28.4 & 28.6 & 77.5 & 85.9 & 71.9 & 45.8 & 79.4 & 87.5 & 41.0 \\
\hline 10.54 & 90.7 & 93.9 & 32.4 & 28.8 & 68.3 & 63.1 & 100.0 & 46.1 & 80.5 & 89.2 & 40.1 \\
\hline 10.7 & 87.2 & 94.0 & 36.0 & 29.1 & 63.6 & & 121.3 & 46.3 & 83.7 & 87.5 & 37.6 \\
\hline 10.88 & 84.2 & 95.2 & 39.2 & 29.3 & 58.3 & & 127.9 & 46.5 & 78.6 & 80.0 & 43.1 \\
\hline 11.09 & 90.1 & 94.3 & 32.6 & 29.5 & 56.2 & & 126.1 & 46.7 & 81.2 & 81.9 & 41.1 \\
\hline 11.32 & 90.7 & 95.1 & 33.0 & 29.7 & 55.4 & & 114.4 & 46.6 & 84.8 & 87.5 & 36.7 \\
\hline 11.8 & 82.7 & 95.0 & 41.4 & 29.9 & 59.8 & 61.5 & 130.9 & 46.8 & 86.5 & 91.2 & 36.3 \\
\hline 12.04 & 90.6 & 92.2 & 30.3 & 30.1 & 68.6 & & 104.9 & 47 & 88.0 & 91.1 & 31.5 \\
\hline 12.2 & 98.1 & 92.7 & 25.5 & 30.3 & 73.6 & 72.2 & 70.4 & 47.2 & 81.5 & 88.9 & 39.5 \\
\hline 12.38 & 86.3 & 93.5 & 38.5 & 30.6 & 80.0 & 80.9 & 57.7 & 47.4 & 81.5 & 89.4 & 39.8 \\
\hline \multirow[t]{2}{*}{12.59} & 86.1 & 91.4 & 37.2 & 30.8 & 79.1 & 80.5 & 61.6 & 47.6 & 87.5 & 89.4 & 31.7 \\
\hline & & & & 31 & 79.4 & 78.7 & 69.1 & 47.8 & 89.1 & 91.6 & 29.4 \\
\hline 12.82 & 89.0 & 92.9 & 33.1 & 31.2 & 81.6 & 83.4 & 62.4 & 48.1 & 91.2 & 92.6 & 27.7 \\
\hline 13.12 & 77.1 & 85.5 & 54.0 & 31.4 & 89.9 & 87.9 & 30.7 & 48.3 & 88.1 & 92.1 & 32.6 \\
\hline 13.3 & 68.4 & 84.6 & 80.9 & 31.6 & 83.8 & 83.7 & 47.6 & 48.5 & 77.3 & 87.8 & 43.9 \\
\hline 13.54 & 61.2 & 81.3 & 102.3 & 31.8 & 79.8 & 86.4 & 52.8 & 48.7 & 87.1 & 90.8 & 31.8 \\
\hline 13.7 & 66.3 & 80.0 & 81.1 & 32.1 & 83.2 & 87.8 & 45.7 & 48.9 & 85.6 & 91.8 & 34.2 \\
\hline 13.88 & 70.2 & 87.5 & 73.6 & 32.3 & 88.9 & 90.2 & 38.6 & 49.1 & 90.0 & 91.7 & 27.4 \\
\hline 14.09 & 78.1 & 89.1 & 51.5 & 32.5 & 84.3 & 86.8 & 38.3 & 49.3 & 91.0 & 92.4 & 28.6 \\
\hline 14.34 & 72.0 & 86.2 & 68.5 & 32.7 & 85.4 & 85.1 & 38.2 & 49.6 & 86.3 & 92.5 & 33.3 \\
\hline 14.62 & 76.9 & 86.8 & 60.4 & 32.9 & 81.2 & 80.7 & 37.8 & 49.8 & 84.2 & 91.0 & 36.5 \\
\hline 14.8 & 84.7 & 90.4 & 44.2 & 33.1 & 93.4 & 94.5 & 24.1 & 50 & 84.5 & 89.9 & 34.7 \\
\hline 15.04 & 74.2 & 86.6 & 59.4 & 33.3 & 88.4 & 91.4 & 30.2 & 50.2 & 82.1 & 89.0 & 38.1 \\
\hline 15.2 & 68.1 & 85.2 & 102.3 & 33.6 & 78.3 & 83.9 & 42.8 & 50.4 & 85.9 & 88.9 & 35.4 \\
\hline 15.38 & 78.2 & 86.8 & 57.2 & 33.8 & 78.9 & 83.1 & 46.0 & 50.6 & 83.8 & 87.2 & 36.3 \\
\hline 15.59 & 84.1 & 88.4 & 44.5 & 34 & 77.1 & 80.3 & 53.3 & 50.8 & 83.4 & 87.9 & 36.6 \\
\hline 15.82 & 79.1 & 87.7 & 52.1 & 34.2 & 71.9 & 81.1 & 51.4 & 51.1 & 85.6 & 88.9 & 34.6 \\
\hline 16.12 & 81.7 & 86.8 & 57.8 & 34.4 & 73.3 & 82.2 & 49.0 & 51.3 & 85.0 & 88.5 & 35.4 \\
\hline 16.3 & 82.1 & 86.4 & 45.5 & 34.6 & 75.0 & 82.1 & 49.1 & 51.5 & 85.5 & 90.7 & 35.6 \\
\hline 16.54 & 73.8 & 84.1 & 59.8 & 34.8 & 90.1 & 90.5 & 24.9 & 51.7 & 85.9 & 88.5 & 33.7 \\
\hline 16.7 & 73.9 & 84.3 & 64.0 & 35.1 & 82.7 & 85.4 & 37.3 & 51.9 & 86.1 & 89.1 & 33.5 \\
\hline 16.88 & 76.7 & 84.1 & 58.9 & 35.3 & 88.2 & 89.6 & 31.4 & 52.1 & 87.0 & 88.2 & 33.2 \\
\hline 17.09 & 61.2 & 75.8 & 123.9 & 35.5 & 82.8 & $\begin{array}{l}89.0 \\
87.7\end{array}$ & 38.5 & 52.3 & 79.9 & 85.7 & 41.2 \\
\hline 17.32 & 77.8 & 86.7 & 58.0 & 35.7 & 84.6 & 89.8 & 35.4 & 52.6 & 81.3 & 87.9 & 34.6 \\
\hline 17.57 & 72.2 & 100.0 & 70.0 & 35.9 & 83.0 & 89.3 & 37.5 & 52.8 & 80.7 & 86.5 & 42.0 \\
\hline 17.77 & 79.7 & 85.4 & 53.0 & 36.1 & 84.5 & 86.9 & 36.2 & 53 & 84.7 & 87.3 & 37.3 \\
\hline 18.04 & 76.1 & 86.5 & 56.5 & 36.3 & 83.6 & 87.3 & 37.2 & 53.2 & 84.6 & 87.4 & 37.2 \\
\hline 18.1 & 81.2 & 90.6 & 46.8 & 36.6 & 86.4 & 86.8 & 29.9 & 53.4 & 80.2 & 87.1 & 40.2 \\
\hline 18.3 & 88.4 & 91.5 & 35.5 & 36.8 & 68.0 & 85.5 & 56.2 & 53.6 & 84.8 & 87.3 & 36.5 \\
\hline 18.52 & 87.0 & 92.8 & 34.5 & 37 & 78.6 & 84.5 & 42.4 & 53.8 & 81.6 & 85.7 & 39.7 \\
\hline 18.7 & 87.5 & 91.2 & 36.0 & 37.2 & 78.7 & 82.3 & 41.9 & 54.1 & 85.0 & 88.0 & 36.7 \\
\hline
\end{tabular}


Table 3 (continued).

\begin{tabular}{|c|c|c|c|c|c|c|c|c|c|c|c|}
\hline $\begin{array}{l}\text { Sample } \\
\text { depth } \\
\text { (mbsf) }\end{array}$ & $\begin{array}{c}\text { A } \\
\% \text { fine } \\
\text { fraction } \\
(<63 \mu \mathrm{m})\end{array}$ & $\begin{array}{c}\text { B } \\
\% \text { fine } \\
\text { fraction } \\
(<63 \mu \mathrm{m})\end{array}$ & $\begin{array}{c}\text { Mean } \\
\text { particle size } \\
(\mu \mathrm{m})\end{array}$ & $\begin{array}{l}\text { Sample } \\
\text { depth } \\
\text { (mbsf) }\end{array}$ & $\begin{array}{c}\text { A } \\
\% \text { fine } \\
\text { fraction } \\
(<63 \mu \mathrm{m})\end{array}$ & $\begin{array}{c}\text { B } \\
\% \text { fine } \\
\text { fraction } \\
(<63 \mu \mathrm{m})\end{array}$ & $\begin{array}{c}\text { Mean } \\
\text { particle size } \\
(\mu \mathrm{m})\end{array}$ & $\begin{array}{l}\text { Sample } \\
\text { depth } \\
\text { (mbsf) }\end{array}$ & $\begin{array}{c}\text { A } \\
\% \text { fine } \\
\text { fraction } \\
(<63 \mu \mathrm{m})\end{array}$ & $\begin{array}{c}\text { B } \\
\% \text { fine } \\
\text { fraction } \\
(<63 \mu \mathrm{m})\end{array}$ & $\begin{array}{c}\text { Mean } \\
\text { particle size } \\
(\mu \mathrm{m})\end{array}$ \\
\hline 54.3 & 82.9 & 86.4 & 39.8 & 73.02 & 76.8 & 91.9 & 45.0 & 90.5 & 89.8 & 95.0 & 28.9 \\
\hline 54.5 & 82.7 & 86.3 & 39.5 & 73.22 & 77.8 & 86.6 & 43.4 & 91 & 87.1 & 92.9 & 31.3 \\
\hline 54.7 & 85.9 & 87.4 & 36.0 & 73.42 & 86.3 & 89.5 & 33.4 & 91.2 & 83.4 & 90.4 & 34.6 \\
\hline 54.9 & 82.9 & 84.5 & 38.6 & 73.72 & 90.0 & 89.3 & 26.2 & 91.4 & 82.7 & 94.1 & 35.2 \\
\hline 55.1 & 82.9 & 85.9 & 38.9 & 73.92 & 85.8 & 91.3 & 29.8 & 91.6 & 87.2 & 92.2 & 28.7 \\
\hline 55.3 & 81.6 & 87.7 & 39.4 & 74.12 & 86.9 & 92.1 & 27.8 & 91.8 & 70.0 & 79.8 & 47.7 \\
\hline 56.1 & 87.0 & 85.1 & 33.8 & 74.32 & 83.3 & 90.8 & 32.1 & 92 & 84.1 & 90.6 & 32.8 \\
\hline 56.3 & 84.2 & 86.7 & 36.7 & 74.52 & 78.4 & 90.0 & 36.4 & 92.2 & 89.2 & 93.0 & 27.1 \\
\hline 56.5 & 76.7 & 82.0 & 45.3 & 74.72 & 88.7 & 91.4 & 27.6 & 92.5 & 86.6 & 92.6 & 31.6 \\
\hline 56.7 & 78.1 & 86.2 & 38.7 & 74.92 & 84.3 & 93.3 & 30.8 & 92.7 & 88.6 & 93.5 & 27.1 \\
\hline 56.9 & 83.0 & 92.1 & 35.3 & 75.1 & 79.6 & 87.2 & 36.6 & 92.9 & 91.1 & 94.9 & 26.2 \\
\hline 57.1 & 81.2 & 88.5 & 35.4 & 75.3 & 87.7 & 92.2 & 27.4 & 93.1 & 88.1 & 91.5 & 28.2 \\
\hline 57.3 & 82.8 & 90.4 & 35.2 & 75.45 & 94.6 & 94.7 & 22.3 & 93.3 & 88.7 & 90.6 & 27.7 \\
\hline 57.6 & 80.5 & 89.2 & 39.1 & 75.65 & 90.3 & 94.3 & 24.9 & 93.5 & 85.8 & 93.2 & 30.4 \\
\hline 57.8 & 88.7 & 91.0 & 29.8 & 75.85 & 92.0 & 92.9 & 24.5 & 93.7 & 87.7 & 92.7 & 29.0 \\
\hline 58 & 80.8 & 89.1 & 36.5 & 76.05 & 92.6 & 94.0 & 23.5 & 94 & 86.5 & 93.4 & 28.9 \\
\hline 58.2 & 81.0 & 89.8 & 37.2 & 76.25 & 87.3 & 90.1 & 27.5 & 94.2 & 91.3 & 93.1 & 25.0 \\
\hline 58.4 & 86.2 & 88.9 & 32.2 & 76.45 & 90.5 & 93.0 & 25.3 & 94.4 & 81.2 & 93.6 & 34.1 \\
\hline 58.6 & 80.1 & 88.4 & 37.5 & 76.65 & 89.9 & 92.1 & 25.1 & 94.6 & 84.7 & 87.4 & 31.2 \\
\hline 58.8 & 76.9 & 81.7 & 40.2 & 76.95 & 89.5 & 92.9 & 26.5 & 94.1 & 87.6 & 93.6 & 28.0 \\
\hline 59.1 & 86.8 & 90.5 & 31.2 & 77.15 & 89.6 & 93.2 & 25.9 & 94.42 & 90.0 & 91.2 & 25.3 \\
\hline 59.3 & 81.4 & 85.7 & 36.7 & 77.35 & 81.3 & 91.7 & 34.1 & 94.62 & 79.7 & 89.2 & 36.2 \\
\hline 59.5 & 84.2 & 88.5 & 33.1 & 78.15 & 85.3 & 90.4 & 30.7 & 94.82 & 80.0 & 92.6 & 36.2 \\
\hline 59.7 & 85.0 & 87.2 & 35.6 & 78.45 & 88.9 & 90.9 & 27.1 & 95.02 & 89.0 & 93.9 & 25.9 \\
\hline 59.9 & 85.9 & 87.8 & 33.7 & 78.65 & 75.4 & 90.0 & 41.2 & 95.22 & 85.4 & 91.9 & 29.9 \\
\hline 60.1 & 83.5 & 89.3 & 34.5 & 78.85 & 85.5 & 91.0 & 31.2 & 95.42 & 85.9 & 90.2 & 29.6 \\
\hline 60.3 & 88.4 & 89.0 & 30.1 & 79.05 & 82.9 & 92.9 & 33.2 & 95.62 & 83.9 & 91.2 & 31.6 \\
\hline 60.6 & 85.3 & 90.8 & 35.8 & 79.25 & 77.3 & 89.1 & 38.0 & 95.92 & 87.1 & 92.3 & 28.2 \\
\hline 60.8 & 86.1 & 92.2 & 32.8 & 79.45 & 86.0 & 91.7 & 28.9 & 96.12 & 88.8 & 92.4 & 27.0 \\
\hline 61 & 84.2 & 91.0 & 37.7 & 79.65 & 90.7 & 94.6 & 25.8 & 96.32 & 84.2 & 91.9 & 31.1 \\
\hline 61.2 & 86.9 & 90.0 & 30.7 & 79.95 & 82.4 & 88.4 & 33.6 & 96.52 & 80.7 & 88.0 & 35.6 \\
\hline 61.4 & 84.7 & 92.2 & 36.8 & 80.15 & 80.6 & 89.8 & 38.4 & 96.72 & 85.8 & 90.6 & 30.4 \\
\hline 61.6 & 88.8 & 91.4 & 29.7 & 80.35 & 86.1 & 88.3 & 30.4 & 96.92 & 84.2 & 90.2 & 31.5 \\
\hline 61.8 & 88.8 & 92.9 & 28.6 & 80.55 & 82.5 & 89.3 & 34.1 & 97.12 & 86.0 & 91.8 & 29.1 \\
\hline 62.1 & 87.8 & 88.1 & 30.7 & 80.75 & 82.5 & 87.5 & 34.7 & 97.42 & 90.4 & 91.9 & 25.1 \\
\hline 62.3 & 87.3 & 92.9 & 31.2 & 80.95 & 82.7 & 88.8 & 35.0 & 97.62 & 85.7 & 91.2 & 29.9 \\
\hline 62.5 & 78.1 & 88.5 & 45.1 & 81.15 & 89.3 & 89.0 & 27.2 & 97.82 & 85.5 & 90.4 & 30.5 \\
\hline 62.7 & 90.9 & 95.0 & 29.8 & 81.45 & 88.3 & 90.6 & 27.7 & 98.02 & 87.9 & 92.0 & 27.5 \\
\hline 62.9 & 85.1 & 91.9 & 37.3 & 81.65 & 85.8 & 90.4 & 32.0 & 98.22 & 84.7 & 90.3 & 30.9 \\
\hline 63.1 & 89.0 & 91.2 & 28.9 & 81.85 & 89.8 & 90.5 & 27.1 & 98.42 & 85.9 & 90.6 & 30.6 \\
\hline 63.6 & 88.4 & 91.3 & 28.8 & 82.05 & 89.4 & 89.3 & 26.9 & 98.62 & 84.5 & 88.4 & 33.6 \\
\hline 63.8 & 90.7 & 93.3 & 30.3 & 82.25 & 91.1 & 91.8 & 25.8 & 98.92 & 87.9 & 90.8 & 29.4 \\
\hline 64 & 82.9 & 89.7 & 34.5 & 82.45 & 90.9 & 91.7 & 25.3 & 99.12 & 91.9 & 93.1 & 24.2 \\
\hline 64.2 & 87.3 & 93.1 & 30.8 & 82.65 & 80.1 & 90.5 & 38.1 & 99.32 & 89.2 & 87.3 & 26.9 \\
\hline 64.4 & 90.2 & 92.8 & 29.9 & 83.95 & 85.9 & 90.4 & 31.8 & 99.52 & 83.8 & 89.2 & 32.7 \\
\hline 64.6 & 91.7 & 94.0 & 26.8 & 83.15 & 86.5 & 92.4 & 31.3 & 99.72 & 90.7 & 92.8 & 25.2 \\
\hline 64.8 & 87.8 & 93.9 & 33.3 & 83.35 & 88.1 & 86.1 & 28.2 & 99.92 & 87.4 & 89.6 & 29.2 \\
\hline 65.1 & 89.2 & 90.7 & 33.6 & 83.55 & 92.6 & 90.1 & 27.3 & 100.12 & 86.2 & 89.1 & 30.3 \\
\hline 65.3 & 86.2 & 90.5 & 34.6 & 83.75 & 90.3 & 92.0 & 26.5 & 100.42 & 85.3 & 86.1 & 31.2 \\
\hline 65.5 & 83.9 & 86.8 & 37.9 & 83.95 & 85.1 & 89.6 & 32.1 & 100.62 & 87.6 & 88.0 & 30.0 \\
\hline 65.7 & 74.9 & 87.0 & 43.9 & 84.15 & 81.8 & 89.8 & 35.7 & 100.82 & 89.6 & 89.2 & 27.3 \\
\hline 65.6 & 69.3 & 77.3 & 61.1 & 84.45 & 85.7 & 89.3 & 30.9 & 101.02 & 85.2 & 89.3 & 31.6 \\
\hline 65.8 & 64.8 & 77.3 & 58.5 & 84.65 & 82.1 & 89.4 & 35.5 & 101.22 & 86.2 & 89.6 & 30.5 \\
\hline 66 & 79.1 & 84.9 & 38.1 & 84.85 & 91.4 & 91.4 & 24.8 & 101.42 & 86.3 & 87.0 & 30.4 \\
\hline 66.22 & 67.6 & 83.3 & 51.7 & 85.05 & 88.7 & 90.3 & 29.6 & 101.62 & 85.8 & 87.8 & 31.8 \\
\hline 66.42 & 72.5 & 81.8 & 47.5 & 84.6 & 79.5 & 86.2 & 39.3 & 101.92 & 89.7 & 91.2 & 28.2 \\
\hline 66.62 & 77.3 & 87.2 & 40.6 & 84.8 & 90.2 & 92.1 & 25.9 & 102.12 & 88.1 & 85.3 & 29.2 \\
\hline 66.82 & 84.9 & 86.1 & 37.7 & 85 & 91.6 & 93.4 & 25.7 & 102.32 & 84.5 & 88.3 & 32.8 \\
\hline 67.02 & 81.2 & 88.9 & 36.9 & 85.2 & 87.5 & 92.1 & 29.0 & 102.52 & 88.7 & 90.7 & 28.3 \\
\hline 67.22 & 83.5 & 87.4 & 33.3 & 85.4 & 85.8 & 90.9 & 30.7 & 102.72 & 82.4 & 87.3 & 34.3 \\
\hline 67.42 & 79.9 & 87.8 & 36.8 & 85.6 & 89.3 & 91.8 & 26.9 & 102.92 & 82.0 & 88.3 & 35.3 \\
\hline 67.72 & 73.9 & 89.1 & 43.0 & 85.8 & 84.1 & 88.3 & 32.6 & 103.12 & 82.4 & 89.1 & 35.0 \\
\hline 67.92 & 65.9 & 80.9 & 52.1 & 86 & 72.7 & 82.1 & 43.3 & 103.42 & 82.0 & 86.1 & 35.3 \\
\hline 68.12 & 77.5 & 86.8 & 40.3 & 86.2 & 88.5 & 91.9 & 29.1 & 103.62 & 86.1 & 88.3 & 30.4 \\
\hline 68.92 & 75.6 & 85.4 & 47.1 & 86.5 & 90.3 & 42.4 & 27.8 & 103.82 & 84.8 & 89.2 & 31.9 \\
\hline 69.22 & 70.3 & 87.9 & 47.2 & 86.7 & 75.5 & 87.3 & 41.1 & 104.02 & 82.1 & 85.7 & 35.2 \\
\hline 69.42 & 67.6 & 79.8 & 56.7 & 86.9 & 86.9 & 89.8 & 31.1 & 103.6 & 78.9 & 85.9 & 39.1 \\
\hline 69.62 & 68.5 & 77.4 & 48.4 & 87.1 & 77.0 & 87.6 & 40.2 & 103.8 & 76.9 & 86.6 & 39.6 \\
\hline 69.82 & 72.2 & 85.6 & 51.4 & 87.3 & 75.9 & 88.7 & 41.0 & $104^{\circ}$ & 77.7 & 86.3 & 39.5 \\
\hline 70.02 & 55.6 & 75.5 & 63.2 & 87.5 & 86.4 & 90.4 & 30.9 & 104.2 & 81.4 & 85.6 & 35.7 \\
\hline 70.22 & 68.5 & 83.2 & 55.1 & 87.7 & 81.8 & 90.3 & 35.8 & 104.4 & & & \\
\hline 70.42 & 81.2 & 88.6 & 36.4 & 88 & 83.6 & 90.3 & 34.4 & 105.1 & 75.0 & 84.7 & 42.4 \\
\hline 70.72 & 84.7 & 86.5 & 33.0 & 88.2 & 86.1 & 92.0 & 32.0 & 105.3 & 74.4 & 84.8 & 43.6 \\
\hline 70.92 & 66.4 & 85.9 & 49.9 & 88.4 & 86.4 & 91.7 & 32.6 & 105.5 & 76.8 & 84.5 & 40.3 \\
\hline 71.12 & 79.0 & 88.7 & 43.3 & 88.6 & 89.7 & 93.7 & 28.8 & 105.7 & 79.9 & 82.3 & 37.2 \\
\hline 71.32 & 82.6 & 91.3 & 38.4 & 88.8 & 90.7 & 90.6 & 29.9 & 105.9 & 72.3 & 80.2 & 45.0 \\
\hline 71.52 & 85.1 & 92.0 & 32.5 & 89 & 91.5 & 92.3 & 28.5 & 106.1 & 68.9 & 73.1 & 47.6 \\
\hline 71.72 & 84.7 & 91.8 & 32.1 & 89.2 & 85.3 & 95.2 & 29.8 & 106.3 & 72.5 & 78.7 & 44.4 \\
\hline 71.92 & 88.9 & 91.4 & 30.0 & 89.5 & 66.3 & 90.4 & 50.7 & 106.6 & 74.0 & 82.1 & 42.5 \\
\hline 72.22 & 81.8 & 91.9 & 39.2 & 89.7 & 92.8 & 95.3 & 26.6 & 106.8 & 77.3 & 82.6 & 40.0 \\
\hline 72.42 & 82.3 & 92.9 & 38.7 & 89.9 & 86.2 & 93.0 & 32.8 & 107 & 75.9 & 80.2 & 41.1 \\
\hline 72.62 & 83.7 & 87.7 & 36.9 & 90.1 & 93.0 & 96.0 & 26.1 & 107.2 & 64.2 & 73.5 & 53.9 \\
\hline 72.82 & 86.9 & 90.9 & 33.0 & 90.3 & 91.1 & 93.5 & 27.4 & 107.33 & 66.2 & 69.4 & 54.1 \\
\hline
\end{tabular}


Table 3 (continued).

\begin{tabular}{|c|c|c|c|}
\hline $\begin{array}{c}\text { Sample } \\
\text { depth } \\
\text { (mbsf) }\end{array}$ & $\begin{array}{c}\text { A } \\
\% \text { fine } \\
\text { fraction } \\
(<63 \mu \mathrm{m})\end{array}$ & $\begin{array}{c}\text { B } \\
\% \text { fine } \\
\text { fraction } \\
(<63 \mu \mathrm{m})\end{array}$ & $\begin{array}{c}\text { Mean } \\
\text { particle size } \\
(\mu \mathrm{m})\end{array}$ \\
\hline 107.6 & 74.0 & 76.7 & 43.4 \\
\hline 108.1 & 77.4 & 81.8 & 41.6 \\
\hline 108.3 & 66.7 & 34.9 & 56.7 \\
\hline 108.5 & 82.5 & 79.5 & 33.1 \\
\hline 108.7 & 84.1 & 58.8 & 31.3 \\
\hline 108.9 & 79.4 & 80.4 & 36.4 \\
\hline 109.1 & 76.6 & 73.3 & 44.3 \\
\hline 109.3 & 79.2 & 82.6 & 36.7 \\
\hline 109.6 & 75.6 & 84.2 & 40.6 \\
\hline 109.8 & 77.8 & 82.0 & 38.6 \\
\hline 110 & 83.3 & 19.3 & 33.0 \\
\hline 110.2 & 75.9 & 79.0 & 41.1 \\
\hline 110.4 & 78.2 & 79.8 & 37.9 \\
\hline 110.6 & 73.1 & 80.5 & 45.9 \\
\hline 110.78 & 71.5 & 81.4 & 46.6 \\
\hline 111.1 & 86.0 & 86.7 & 30.7 \\
\hline 111.3 & 87.3 & 89.6 & 30.5 \\
\hline 111.55 & 91.4 & 85.6 & 27.4 \\
\hline 111.68 & 89.3 & 89.9 & 29.4 \\
\hline 111.88 & 87.7 & 88.0 & 30.5 \\
\hline 112.08 & 88.3 & 76.5 & 30.0 \\
\hline 112.28 & 93.8 & 89.8 & 24.1 \\
\hline 112.46 & 85.2 & 90.3 & 32.4 \\
\hline 112.68 & 91.0 & 91.9 & 27.0 \\
\hline 112.88 & 91.2 & 85.8 & 25.9 \\
\hline 113.13 & 88.6 & 83.9 & 28.7 \\
\hline 113.38 & 91.5 & 89.1 & 26.9 \\
\hline 113.58 & 90.5 & 88.8 & 26.1 \\
\hline 113.78 & 88.2 & 86.7 & 29.6 \\
\hline 113.96 & 87.9 & 89.3 & 29.4 \\
\hline 114.18 & 90.4 & 86.1 & 27.4 \\
\hline 114.38 & 85.3 & 88.2 & 32.3 \\
\hline 114.68 & 82.4 & 87.5 & 35.5 \\
\hline 114.88 & 88.5 & 88.6 & 29.3 \\
\hline 115.08 & 87.4 & 86.7 & 30.6 \\
\hline 115.28 & 79.5 & 83.1 & 41.5 \\
\hline 115.46 & 91.0 & 91.6 & 26.1 \\
\hline 115.68 & 91.7 & 90.5 & 26.1 \\
\hline 115.88 & 88.6 & 89.4 & 30.7 \\
\hline 116.18 & 89.8 & 90.0 & 27.1 \\
\hline 116.38 & 92.3 & 88.8 & 24.6 \\
\hline 116.58 & 89.6 & 88.6 & 27.6 \\
\hline 116.78 & 81.0 & 90.0 & 39.4 \\
\hline 116.96 & 87.6 & 89.2 & 29.8 \\
\hline 117.18 & 83.4 & 90.7 & 35.7 \\
\hline 117.68 & 83.8 & 88.5 & 35.7 \\
\hline 117.88 & 84.9 & 87.2 & 32.6 \\
\hline 118.08 & 79.8 & 85.7 & 37.9 \\
\hline 118.28 & 74.4 & 81.9 & 43.1 \\
\hline 118.46 & 73.6 & 83.0 & 45.0 \\
\hline 118.68 & 72.6 & 78.4 & 44.7 \\
\hline 118.88 & 77.6 & 77.4 & 40.6 \\
\hline 119.18 & 69.5 & 76.2 & 48.3 \\
\hline 119.38 & 67.3 & 78.5 & 49.0 \\
\hline 119.58 & 72.1 & 76.5 & 46.4 \\
\hline 119.78 & 74.3 & 78.9 & 43.6 \\
\hline 119.96 & 70.3 & 80.0 & 46.1 \\
\hline 120.18 & 73.7 & 80.8 & 43.2 \\
\hline
\end{tabular}

Column B data generated using sieving method (see analytical techniques), data in columns $\mathrm{A}$ and mean particle size generated using Coulter particle size analyzer.

(Table 3). Particle size characteristics obtained from the sieving method (see analytical techniques) show a general decrease in mean percentage of fine fraction down the core to about $32.5 \mathrm{mbsf}$. The amplitude of fluctuations of the percentage of fine fraction is significantly higher above rather than below $32.5 \mathrm{mbsf}$ for both sieving and Coulter methods of particle size analysis.

The mean particle size curve for Hole $819 \mathrm{~A}$ (generated by the Coulter LS-100 particle size analyzer) shows that little variation in mean grain size occurs below $32.5 \mathrm{mbsf}$, but considerable variation occurs above 32.5 mbsf.

The relationships between particle grain size distribution and planktonic foraminiferal oxygen isotope ratios are particularly complicated and are illustrated in Figures 9 and 10. Below 32.5 mbsf, no clear relationship exists between these two parameters. However, above $32.5 \mathrm{mbsf}$, the relationship between particle size and planktonic

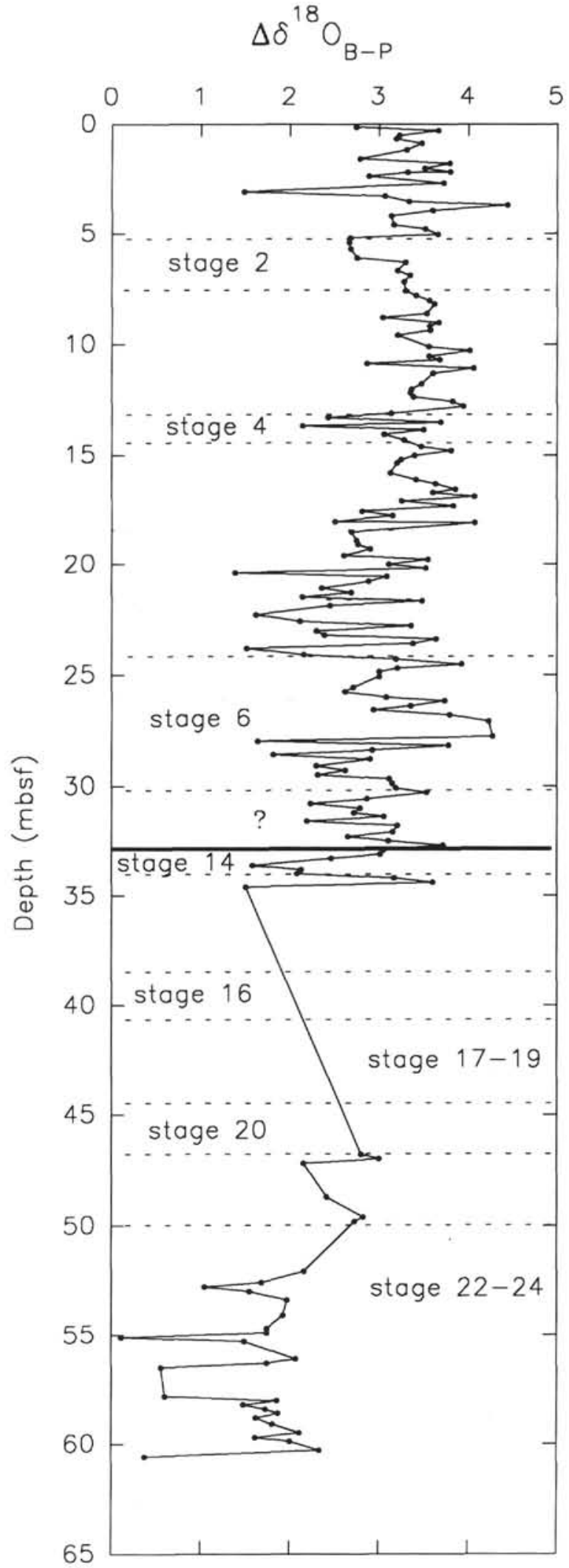

Figure 4. The difference between the planktonic and benthic foraminiferal oxygen isotope records $\left(\Delta \delta^{18} \mathrm{O}_{\mathrm{B}-\mathrm{P}}\right.$ ) plotted vs. depth at Hole $819 \mathrm{~A}$. Note the difference in the mean $\Delta \delta^{18} \mathrm{O}_{\mathrm{B}-\mathrm{P}}$ values above and below the Brunhes/Matuyama boundary at 41 mbsf. 


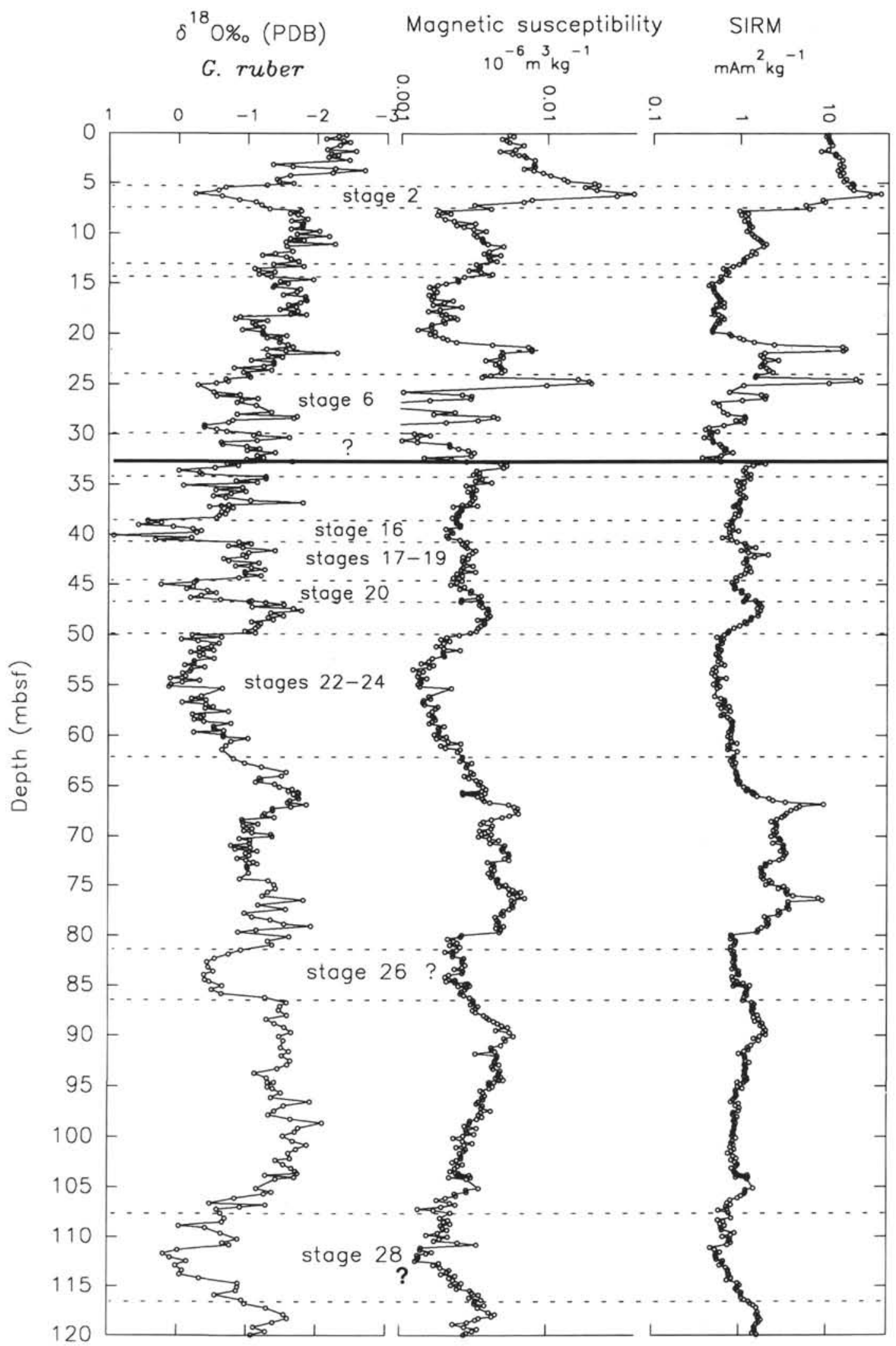

Figure 5. Magnetic mineral measurements ( $X$ and SIRM) and planktonic foraminiferal oxygen isotope record at Hole 819A plotted vs. depth. Note the magnetic relic dissolution layers at 21.7,24.6, 67.2, and $76.2 \mathrm{mbsf}$ and the antithetic relationships among $X$, SIRM, and the oxygen isotope record below the hiatus (solid horizontal line) at $32.5 \mathrm{mbsf}$. Both $X$ and SIRM values were plotted on a common logarithmic scale. 


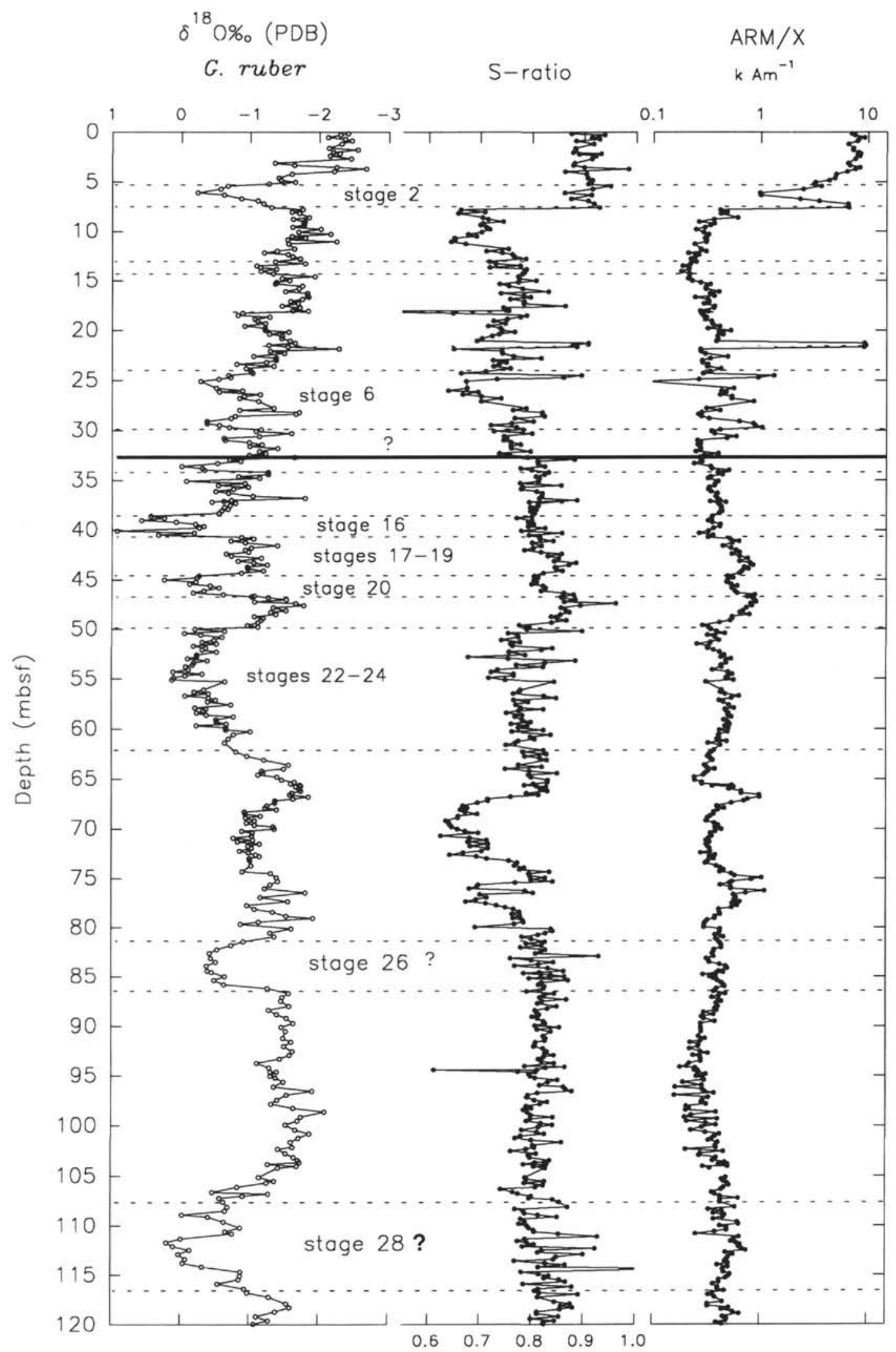

Figure 6. Magnetic mineral ratios (S and ARM/X) and planktonic foraminiferal oxygen isotope record at Hole 819A plotted vs. depth. The solid horizontal line at $32.5 \mathrm{mbsf}$ marks the position of a major stratigraphical hiatus. ARM/X ratios have been plotted on a common logarithmic scale. 

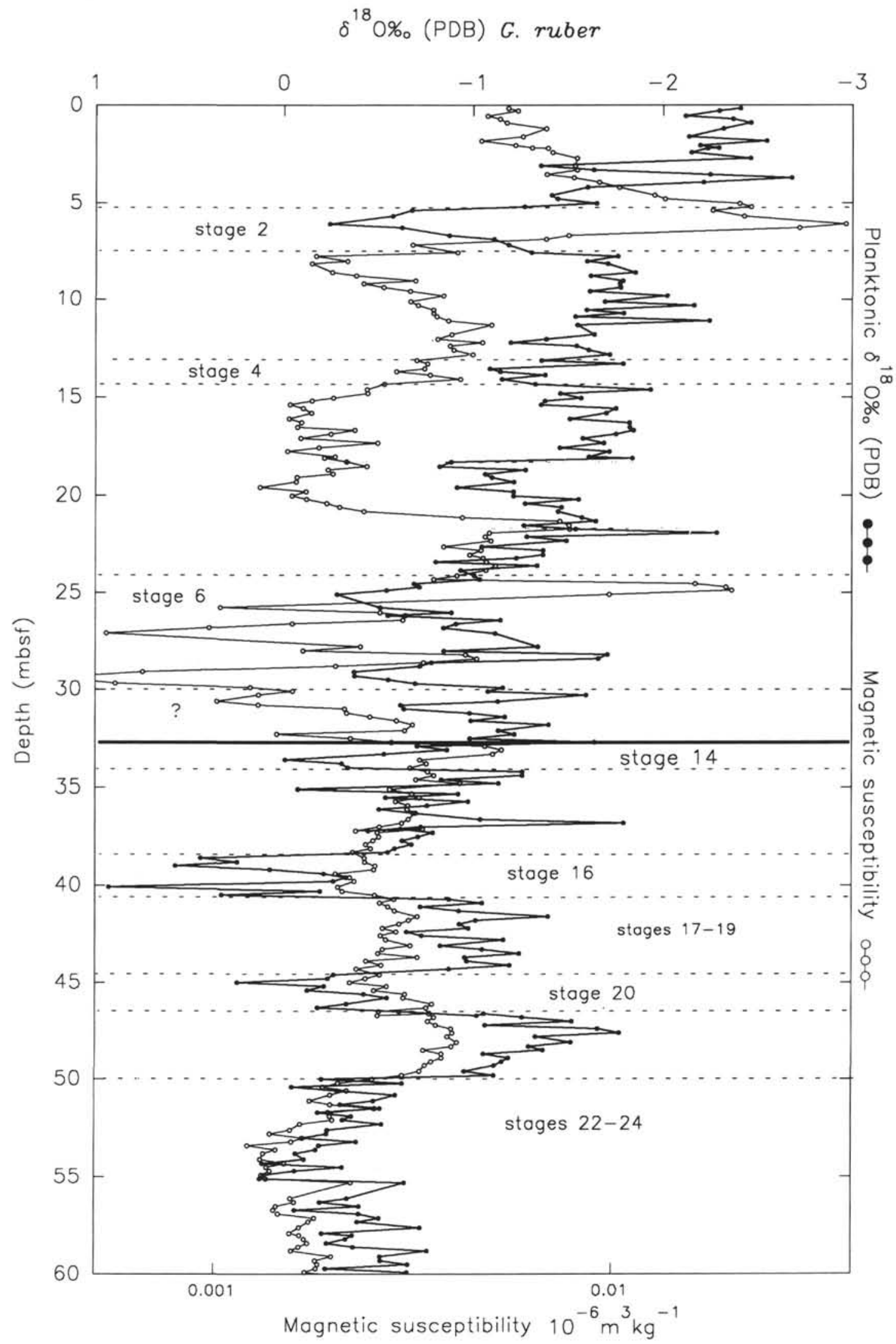

Figure 7. Specific magnetic susceptibility (open circles) and planktonic foraminiferal oxygen isotope record (closed circles) plotted vs. depth at Hole 819A. Note the complex relationship between the records above and below the hiatus (solid horizontal line) at 32.5 mbsf. Magnetic susceptibility values have been plotted on a common logarithmic scale. 


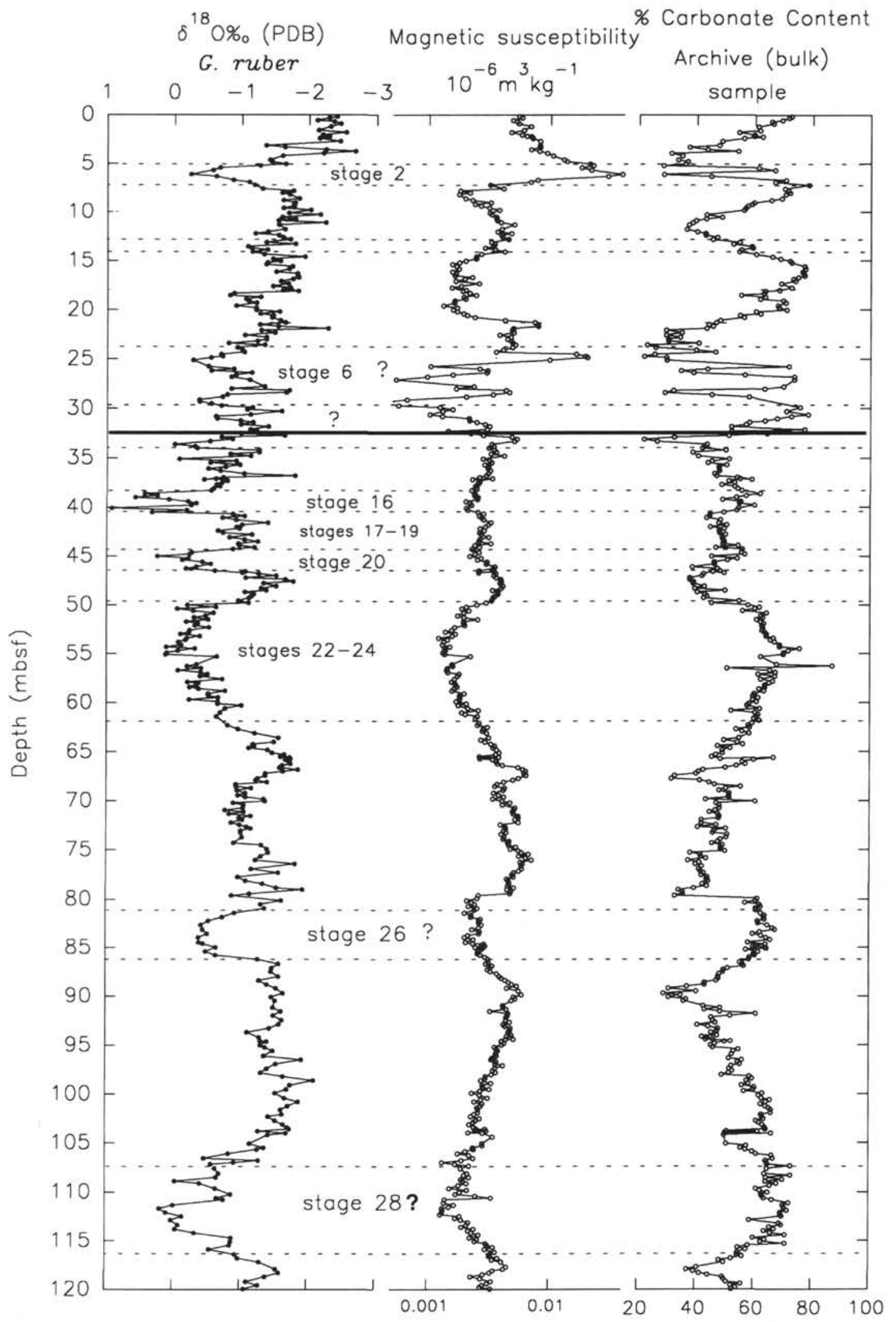

Figure 8. Planktonic foraminiferal oxygen isotope record, magnetic susceptibility, and percentage of carbonate (archive sample) plotted vs. depth. Magnetic susceptibility values have been plotted on a common logarithmic scale. 


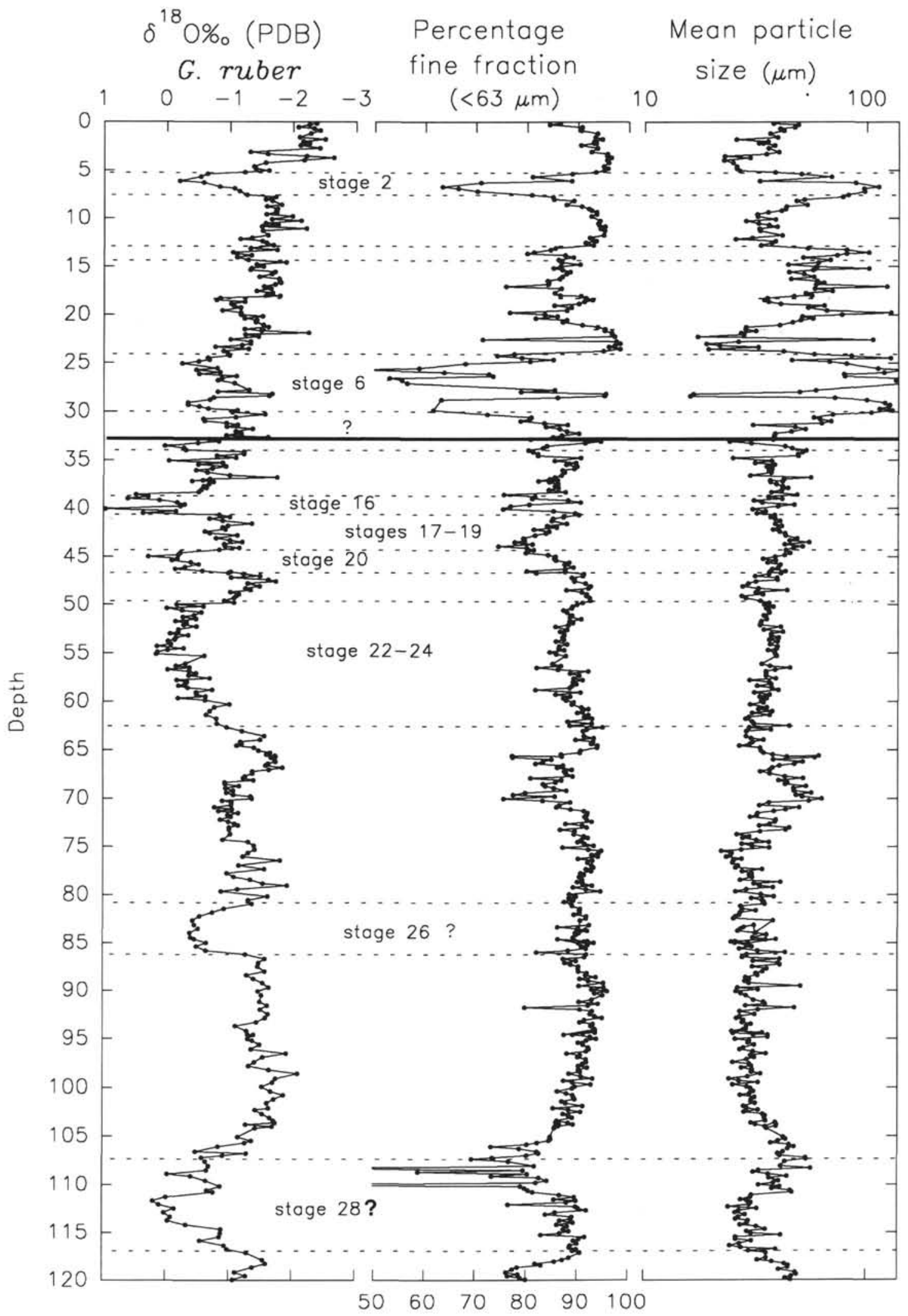

Figure 9. Composite plot showing the percentage of fine fraction (values derived from the wet-sieving method, see analytical techniques) plotted vs. planktonic foraminiferal oxygen isotope record. Note the significant offset of curves at approximately 6 and 25 mbsf. The adjoining plot displays mean particle size values, generated by the Coulter LS-100 counter and shows diminished mean particle size fluctuations below about 32.5 mbsf. 


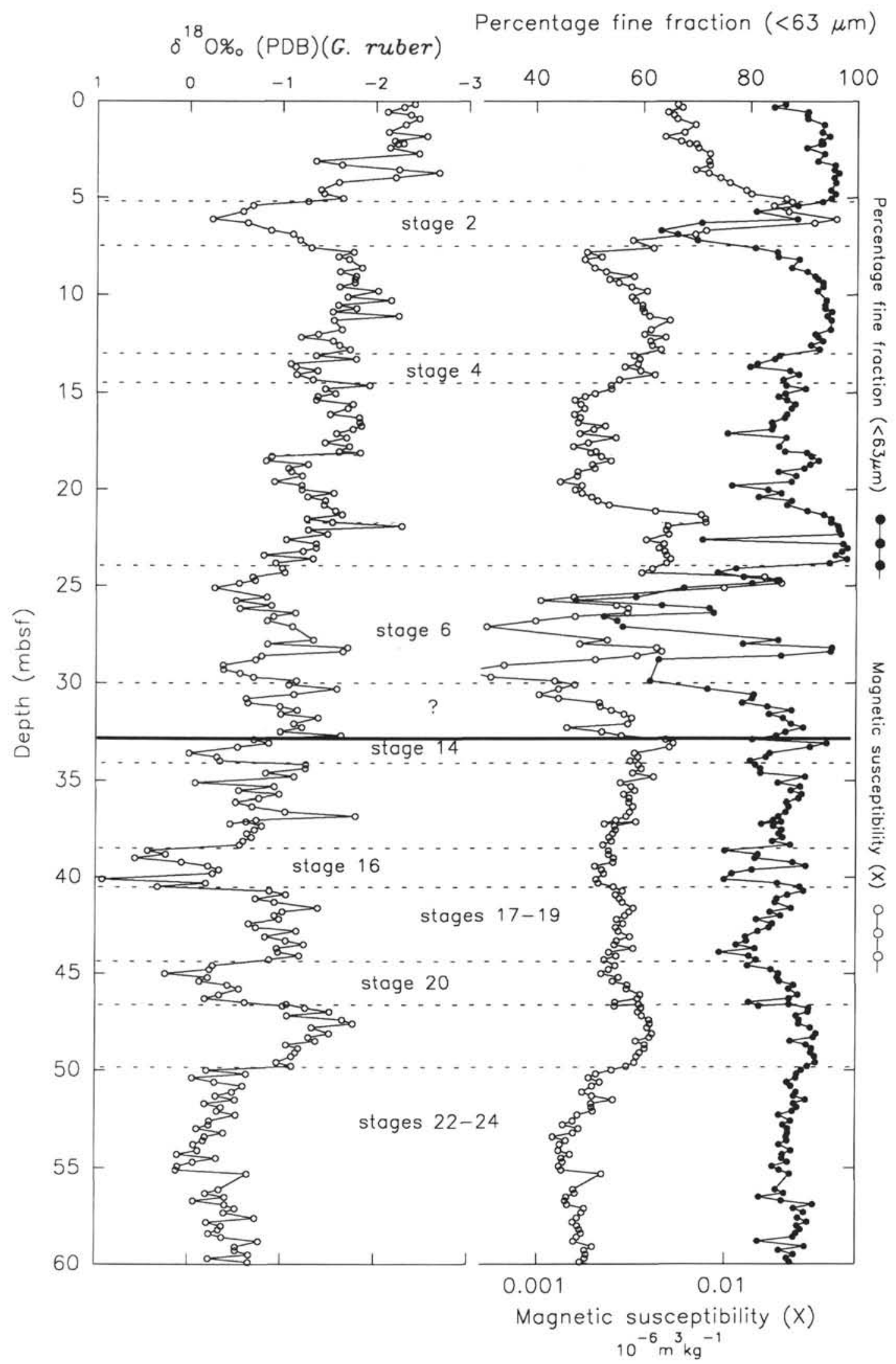

Figure 10. Planktonic foraminiferal oxygen isotope record, percentage of fine fraction $(<63 \mu \mathrm{m}$; closed circles) and magnetic susceptibility (open circles) plotted vs. depth. Magnetic susceptibility values have been plotted on a common logarithmic scale. 
foraminiferal oxygen isotope values is more clearly developed. Above $32.5 \mathrm{mbsf}$, glacial periods were mainly dominated by low percentages of the fine fraction content, while high percentages of the fine fraction content dominate the interglacial periods. Although broadly similar, both particle grain size and planktonic foraminiferal oxygen isotope curves above 32.5 mbsf may be significantly out of phase in places; for example, during stages 2 and 6 , the peak in percentages of the fine fraction clearly occurred before the glacial maxima.

\section{Carbonate Content Determination}

The percentage carbonate content for samples collected at Hole 819 A ranged from $21 \%$ to $87 \%$ (see Fig. 11 and Table 4). The percentages of carbonate content within both the coarse fraction and the archive (bulk) sample, at Hole 819A, display less variation below 32.5 mbsf than above $32.5 \mathrm{mbsf}$. Compared with the archive samples, the coarse fraction samples displayed significantly higher values (by as much as $45 \%$ ) of carbonate content. Below 32.5 mbsf, glacial horizons of the sequence (relative to interglacial horizons) are characterized by a higher percentage of carbonate content. Above $32.5 \mathrm{mbsf}$, relationships between planktonic foraminiferal oxygen isotope values and the percentage of carbonate content are more complex. For example, high percentage of carbonate peaks occur in both stages 5 and 8 . Although the curve for the coarse fraction is broadly similar to the archive sample curve, it does display subtle differences, particularly in the peak positions at $5 \mathrm{mbsf}$ and between 10 to $15 \mathrm{mbsf}$.

The comparison of the percentage of fine fraction with the percentage of carbonate content (Fig. 12), particularly in the upper part of the sequence at Hole 819A, shows that when the fine fraction content is high, the percentage of carbonate content generally is low. Thus, the fine fraction is primarily controlled by detrital influx of a terrigenous origin, resulting in a low carbonate content. A high percentage of fine fraction content generally occurs during early interglacial stages.

\section{DISCUSSION}

The mineral magnetic, particle size, and percentage of carbonate content records all show a major change at $32.5 \mathrm{mbsf}$, which marks the position of a major stratigraphic hiatus. A tentative interpretation of our isotopic data suggests that the hiatus at Hole 819A represents missing marine isotopic stages 10 to 13 (a stratigraphic time gap of about 200 k.y.), or possibly a longer period of time, depending on isotopic stage assignment. The seemingly abrupt change in the nature of sedimentation thus is an artifact of the major hiatus at $32.5 \mathrm{mbsf}$. It is most likely that a gradual or transitional change occurred in the records, between the lower and upper portions of the sequence at Hole 819A.

\section{Mineral Magnetism}

Mineral magnetic characteristics in many marine sediment sequences, for example, on bathymetric highs of the King's Trough area in the North Atlantic (Oldfield and Robinson, 1985; Robinson, 1986) or the Owen Ridge in the Indian Ocean (Bloemendal, 1989) have been found to reflect paleoclimatic changes closely. In these regions, the mineral magnetic variations involve both mineralogical fluctuations and changes in concentration. Mineral magnetic variations often repeat extremely well between neighboring core segments and between drill holes (Bloemendal et al., 1988; Hounslow, 1990). As such, they form the basis of a precise form of lithostratigraphic core correlation. Despite the value of magnetic signatures in core matching and paleoclimatic studies, the cause of magnetic signatures remains poorly understood.

The concentration of magnetic minerals in marine sediments is diluted by any carbonate (or biogenic silica) component (Kent, 1982). Reductive dissolution of iron oxides in the organic-rich upper layers also can profoundly reduce the magnetic content and also change the magnetic grain size (Karlin and Levi, 1983). Diagenesis and authi- genic changes also can produce additional post-depositional magnetic minerals (Henshaw and Merrill, 1980).

For Hole 819A, we propose that the main processes controlling the overall magnetic signature have, in order of importance, been as follows:

1. Dissolution of terrigenous magnetic particles by reductive diagenesis.

2. Dilution by high concentrations of carbonate minerals, and

3. Heavy mineral concentration variations associated with sedimentological particle size changes.

The overriding importance of dissolution at Hole 819A can be seen in the 10-fold decline of magnetic concentration (as measured by, $X$, $\mathrm{ARM}$, or IRM) at a depth of about 7 mbsf (see Figs. 5 and 6). Occasionally, such dissolution processes at Hole 819A have been interrupted, and magnetic mineral relics have been preserved in the sediment sequence. We interpret the magnetic concentration peaks at depths of 21.7, 24.6, 67.2, and 76.2 mbsf as horizons that have escaped dissolution. As described by Karlin and Levi (1983), dissolution changes the grain size of the magnetic minerals as well as the concentration. Such grain size changes clearly are seen in the downcore $\log$ of the $\mathrm{S}$ and $\mathrm{ARM} / \mathrm{X}$ ratios (see Fig. 6). Above 6 mbsf, $\mathrm{S}$ ratios of 0.9 or more were found. These ratios are characteristic of large $(>10 \mu \mathrm{m})$, easily magnetized magnetic grains. Following dissolution below $7 \mathrm{mbsf}, \mathrm{S}$ ratios were reduced to about 0.7 . These lower ratios are typical of stable, single-domain grains having diameters of less than $0.1 \mu \mathrm{m}$ that are more difficult to magnetize (e.g., Thompson, 1986; Dankers, 1978). A likely source of these small magnetite crystals that have survived reductive diagenesis, are inclusions in "dusty" feldspar or other silicate grains (Morgan and Smith, 1981).

A change can be seen in the magnetic characteristics at about 32.5 mbsf. Below this depth, magnetic concentration is inversely related to the $\delta^{18} \mathrm{O}$ ratio. Again, the overall low concentrations are attributable to reductive diagenesis, but the variations that remain in this part of the sequence have been caused by dilution by carbonates (Kent, 1982). This inverse relationship between magnetic concentration and carbonate concentration also holds above the magnetic relic dissolution layers at depths of about 21 to 18 mbsf (see Figs. 5, 6, and 10). Dilution by carbonates, however, is not the only process affecting the magnetic properties in these horizons, because the $\mathrm{S}$ ratio also is changing in parallel with the $\delta^{18} \mathrm{O}$ profile. Low $\mathrm{S}$ ratios of about 0.75 (corresponding to smaller magnetic grain size) are found in the glacial stages, while $\mathrm{S}$ ratios of about 0.9 are found in the interglacial stages. In these deposits, a relationship also has been observed between the particle size distribution ( $\%$ fine fraction) and magnetic concentration (see Figs. 10 and 13). Higher magnetic concentrations are associated with finer-grained sediments. These variations may be explained by changing heavy-mineral fractions that are associated with transportation or by variable dissolution related to changes in the original organic carbon content and productivity.

\section{Present-day Oceanic Circulation}

As we are concerned with the late Quaternary paleoenvironmental history of the continental margin, the question arises of what environmental factors caused the change in the records. The records represent a major shift in the dominant sedimentological regime between the lower and upper parts of the sequence at Hole 819A. Was it a change in the paleoclimate that caused different influxes of sediment, or was it a reorganization of the paleoceanographic circulation, or a combination of these factors? In an attempt to answer these questions, the modern oceanographic setting of Site 819 may provide some clues.

Today's ocean circulation patterns for the western Coral Sea are illustrated in Figure 14 (Pickard et al., 1977; Church, 1987). Surfacewater circulation patterns in the western Coral Sea are largely dominated by the westward-flowing South Equatorial Current (SEC; see 
Table 4. Percentage carbonate content determination for samples collected at Hole 819A.

\begin{tabular}{|c|c|c|c|c|c|c|c|}
\hline $\begin{array}{l}\text { Sample, } \\
\text { interval }(\mathrm{cm})\end{array}$ & $\begin{array}{l}\text { Depth } \\
\text { (mbsf) }\end{array}$ & $\begin{array}{l}\text { \% Carbonate content } \\
\text { Coarse fraction }\end{array}$ & $\begin{array}{l}\text { \% Carbonate content } \\
\text { Archive (bulk) }\end{array}$ & $\begin{array}{c}\text { Sample, } \\
\text { interval }(\mathrm{cm})\end{array}$ & $\begin{array}{l}\text { Depth } \\
\text { (mbsf) }\end{array}$ & $\begin{array}{l}\text { \% Carbonate content } \\
\text { Coarse fraction }\end{array}$ & $\begin{array}{l}\text { \% Carbonate content } \\
\text { Archive (bulk) }\end{array}$ \\
\hline $1 \mathrm{H}-1,16-18$ & 0.16 & 86.4 & 72.4 & $3 \mathrm{H}-2,10-12$ & 19.60 & 89.9 & 67.8 \\
\hline $1 \mathrm{H}-1,30-32$ & 0.30 & 86.9 & 71.6 & $3 \mathrm{H}-2,30-32$ & 19.80 & 87.0 & 68.3 \\
\hline $1 \mathrm{H}-1,54-56$ & 0.54 & 84.7 & 69.3 & $3 \mathrm{H}-2,52-54$ & 20.02 & 87.4 & 70.9 \\
\hline $1 \mathrm{H}-1,70-72$ & 0.70 & 86.7 & 65.6 & $3 \mathrm{H}-2,70-72$ & 20.20 & 81.8 & 60.3 \\
\hline $1 \mathrm{H}-1,90-92$ & 0.90 & 86.3 & 66.2 & $3 \mathrm{H}-2,90-92$ & 20.40 & 78.5 & 61.9 \\
\hline $1 \mathrm{H}-1,120-122$ & 1.20 & 85.9 & 61.0 & $3 \mathrm{H}-2,109-111$ & 20.59 & 80.9 & 54.8 \\
\hline $1 \mathrm{H}-2,10-12$ & 1.60 & 86.2 & 61.5 & $3 \mathrm{H}-2,130-132$ & 20.81 & 79.6 & 56.2 \\
\hline $1 \mathrm{H}-2,30-32$ & 1.80 & 87.8 & 54.4 & $3 \mathrm{H}-3,10-12$ & 21.10 & 71.0 & 48.0 \\
\hline $1 \mathrm{H}-2,54-56$ & 2.04 & 92.1 & 59.7 & $3 \mathrm{H}-3,30-32$ & 21.30 & 74.4 & 46.7 \\
\hline $1 \mathrm{H}-2,68-70$ & 2.18 & 94.9 & 59.4 & $3 \mathrm{H}-3,52-54$ & 21.52 & 72.2 & 44.1 \\
\hline $1 \mathrm{H}-2,70-72$ & 2.20 & 86.2 & 62.5 & $3 \mathrm{H}-3,70-72$ & 21.70 & 82.5 & 45.5 \\
\hline $1 \mathrm{H}-2,90-92$ & 2.40 & 77.4 & 56.1 & $3 \mathrm{H}-3,90-92$ & 21.90 & 88.9 & 43.3 \\
\hline $1 \mathrm{H}-2,120-122$ & 2.70 & 75.6 & 48.6 & $3 \mathrm{H}-3,109-111$ & 22.09 & 71.6 & 29.3 \\
\hline $1 \mathrm{H}-3,10-12$ & 3.10 & 71.1 & 47.5 & $3 \mathrm{H}-3,131-132$ & 22.31 & 70.5 & 34.9 \\
\hline $1 \mathrm{H}-3,30-32$ & 3.30 & 69.9 & 37.3 & $3 \mathrm{H}-4,10-12$ & 22.60 & 46.8 & 29.3 \\
\hline $1 \mathrm{H}-3,54-56$ & 3.54 & 82.2 & 43.6 & $3 \mathrm{H}-4,30-32$ & 22.80 & 67.9 & 34.2 \\
\hline $1 \mathrm{H}-3,70-72$ & 3.70 & 84.4 & 54.1 & $3 \mathrm{H}-4,52-54$ & 23.02 & 57.9 & 30.2 \\
\hline $1 \mathrm{H}-3,95-97$ & 3.95 & 61.1 & 31.1 & $3 \mathrm{H}-4,70-72$ & 23.20 & 64.8 & 30.2 \\
\hline $1 \mathrm{H}-3,120-122$ & 4.20 & 60.9 & 34.9 & $3 \mathrm{H}-4,90-92$ & 23.40 & 46.3 & 40.7 \\
\hline $1 \mathrm{H}-4,10-12$ & 4.60 & 84.2 & 33.3 & $3 \mathrm{H}-4,109-111$ & 23.59 & 70.4 & 22.7 \\
\hline $1 \mathrm{H}-4,30-32$ & 4.80 & 80.3 & 36.7 & $3 \mathrm{H}-4,131-133$ & 23.81 & 62.7 & 25.6 \\
\hline $1 \mathrm{H}-4,54-56$ & 5.04 & 84.1 & 36.0 & $3 \mathrm{H}-5,10-12$ & 24.10 & 74.9 & 39.8 \\
\hline $1 \mathrm{H}-4,70-72$ & 5.20 & 84.2 & 28.4 & $3 \mathrm{H}-5,30-32$ & 24.30 & 69.8 & 46.5 \\
\hline $1 \mathrm{H}-4,90-92$ & 5.40 & 83.0 & 61.2 & $3 \mathrm{H}-5,52-54$ & 24.52 & 61.4 & 25.3 \\
\hline $1 \mathrm{H}-4,120-122$ & 5.70 & 67.6 & 66.8 & $3 \mathrm{H}-5,70-72$ & 24.70 & 46.3 & 21.7 \\
\hline $1 \mathrm{H}-5,10-12$ & 6.10 & 54.5 & 28.5 & $3 \mathrm{H}-5,87-89$ & 24.87 & 58.6 & 29.5 \\
\hline $1 \mathrm{H}-5,30-32$ & 6.30 & 68.8 & 44.8 & $3 \mathrm{H}-5,109-111$ & 25.09 & 76.6 & 29.6 \\
\hline $1 \mathrm{H}-5,70-72$ & 6.70 & 83.1 & 70.6 & $3 \mathrm{H}-6,7-9$ & 25.57 & & \\
\hline $1 \mathrm{H}-5,90-92$ & 6.90 & 84.8 & 66.9 & $3 \mathrm{H}-6,26-28$ & 25.76 & 81.5 & 71.7 \\
\hline $1 \mathrm{H}-5,120-122$ & 7.20 & 86.3 & 78.6 & $3 \mathrm{H}-6,52-54$ & 26.02 & 69.8 & 43.7 \\
\hline $1 \mathrm{H}-6,10-12$ & 7.60 & 88.1 & 70.2 & $3 \mathrm{H}-6,67-69$ & 26.17 & 63.9 & 34.5 \\
\hline $1 \mathrm{H}-6,30-32$ & 7.80 & 87.0 & 71.5 & $3 \mathrm{H}-6,90-92$ & 26.40 & 66.7 & 38.8 \\
\hline $1 \mathrm{H}-6,54-56$ & 8.04 & 87.0 & 72.0 & $3 \mathrm{H}-6,109-111$ & 26.59 & 80.2 & 56.5 \\
\hline $1 \mathrm{H}-6,68-70$ & 8.18 & 83.7 & 70.5 & $3 \mathrm{H}-6,131-133$ & 26.81 & 83.5 & 73.7 \\
\hline $2 \mathrm{H}-1,12-14$ & 8.62 & 86.3 & 69.0 & $3 \mathrm{H}-7,10-12$ & 27.10 & 82.9 & 73.6 \\
\hline $2 \mathrm{H}-1,30-32$ & 8.80 & 86.5 & 65.6 & $4 \mathrm{H}-1,30-32$ & 27.80 & 83.5 & 69.9 \\
\hline $2 \mathrm{H}-1,54-56$ & 9.04 & 82.4 & 59.6 & $4 \mathrm{H}-1,50-52$ & 28.0 & 81.0 & 63.4 \\
\hline $2 \mathrm{H}-1,70-72$ & 9.20 & 88.8 & 58.9 & $4 \mathrm{H}-1,70-72$ & 28.20 & 62.5 & 32.0 \\
\hline $2 \mathrm{H}-1,88-90$ & 9.38 & 84.4 & 57.4 & $4 \mathrm{H}-1,90-92$ & 28.40 & 59.1 & 29.0 \\
\hline $2 \mathrm{H}-1,109-111$ & 9.59 & 82.4 & 56.6 & $4 \mathrm{H}-1,110-112$ & 28.60 & 75.4 & 45.1 \\
\hline $2 \mathrm{H}-1,132-134$ & 9.82 & 81.6 & 56.0 & $4 \mathrm{H}-1,130-132$ & 28.80 & 73.0 & 57.9 \\
\hline $2 \mathrm{H}-2,12-14$ & 10.12 & & & $4 \mathrm{H}-2,10-12$ & 29.10 & & \\
\hline $2 \mathrm{H}-2,30-32$ & 10.03 & 77.3 & 43.2 & $4 \mathrm{H}-2,30-32$ & 29.30 & & \\
\hline $2 \mathrm{H}-2,54-56$ & 10.54 & 81.0 & 48.5 & $4 \mathrm{H}-2,50-52$ & 29.50 & & \\
\hline $2 \mathrm{H}-2,70-72$ & 10.70 & 80.6 & 43.1 & $4 \mathrm{H}-2,70-72$ & 29.70 & & \\
\hline $2 \mathrm{H}-2,88-90$ & 10.88 & 78.6 & 39.5 & $4 \mathrm{H}-2,90-92$ & 29.90 & 82.5 & 75.5 \\
\hline $2 \mathrm{H}-2,109-111$ & 11.09 & 73.3 & 38.5 & $4 \mathrm{H}-2,110-112$ & 30.10 & 85.7 & 67.7 \\
\hline $2 \mathrm{H}-2,132-134$ & 11.32 & 74.4 & 37.1 & $4 \mathrm{H}-2,130-132$ & 30.30 & 85.3 & 70.8 \\
\hline $2 \mathrm{H}-3,30-32$ & 11.80 & 93.7 & 36.4 & $4 \mathrm{H}-3,10-12$ & 30.60 & 86.5 & 78.4 \\
\hline $2 \mathrm{H}-3,54-56$ & 12.04 & 77.3 & 40.0 & $4 \mathrm{H}-3,30-32$ & 30.80 & 86.1 & 72.6 \\
\hline $2 \mathrm{H}-3,70-72$ & 12.20 & 82.3 & 43.0 & $4 \mathrm{H}-3,50-52$ & 31.0 & 85.7 & 67.7 \\
\hline $2 \mathrm{H}-3,88-90$ & 12.38 & 81.6 & 43.0 & $4 \mathrm{H}-3,70-72$ & 31.20 & 88.3 & 63.4 \\
\hline $2 \mathrm{H}-3,109-111$ & 12.59 & 75.0 & 47.0 & $4 \mathrm{H}-3,90-92$ & 31.40 & 86.5 & 58.2 \\
\hline $2 \mathrm{H}-3,132-134$ & 12.82 & 81.8 & 45.4 & $4 \mathrm{H}-3,110-112$ & 31.60 & 86.0 & 57.0 \\
\hline $2 \mathrm{H}-4,12-14$ & 13.12 & 88.6 & 54.7 & $4 \mathrm{H}-3,130-132$ & 31.80 & 87.5 & 51.9 \\
\hline $2 \mathrm{H}-4,30-32$ & 13.30 & 71.9 & 52.5 & $4 \mathrm{H}-4,10-12$ & 32.10 & 90.7 & 77.3 \\
\hline $2 \mathrm{H}-4,54-56$ & 13.54 & 89.5 & 58.8 & $4 \mathrm{H}-4,30-32$ & 32.30 & 89.1 & 52.0 \\
\hline $2 \mathrm{H}-4,70-72$ & 13.70 & 91.4 & 59.1 & $4 \mathrm{H}-4,50-52$ & 32.50 & 87.2 & 64.2 \\
\hline $2 \mathrm{H}-4,88-90$ & 13.88 & 89.8 & 55.2 & $4 \mathrm{H}-4,70-72$ & 32.70 & 80.2 & 51.1 \\
\hline $2 \mathrm{H}-4,109-111$ & 14.09 & 91.5 & 54.5 & $4 \mathrm{H}-4,90-92$ & 32.90 & 54.1 & 32.2 \\
\hline $2 \mathrm{H}-4,132-134$ & 14.34 & 90.5 & 60.3 & $4 \mathrm{H}-4,110-112$ & 33.10 & 51.4 & 21.7 \\
\hline $2 \mathrm{H}-5,12-14$ & 14.62 & 89.0 & 65.9 & $4 \mathrm{H}-4,130-132$ & 33.30 & 50.6 & 26.3 \\
\hline $2 \mathrm{H}-5,30-32$ & 14.80 & 91.7 & 68.7 & $4 \mathrm{H}-5,10-12$ & 33.60 & 55.3 & 43.5 \\
\hline $2 \mathrm{H}-5,54-56$ & 15.04 & 92.5 & 72.0 & $4 \mathrm{H}-5,30-32$ & 33.80 & 57.8 & 42.6 \\
\hline $2 \mathrm{H}-5,70-72$ & 15.20 & 93.3 & 72.6 & $4 \mathrm{H}-5,50-52$ & 34.0 & 55.6 & 41.9 \\
\hline $2 \mathrm{H}-5,88-90$ & 15.38 & & & $4 \mathrm{H}-5,70-72$ & 34.20 & 59.3 & 50.0 \\
\hline $2 \mathrm{H}-5,109-111$ & 15.59 & 92.9 & 77.0 & $4 \mathrm{H}-5,90-92$ & 34.40 & 57.6 & 38.6 \\
\hline $2 \mathrm{H}-5,132-134$ & 15.82 & 91.8 & 77.3 & $4 \mathrm{H}-5,110-112$ & 34.60 & & \\
\hline $2 \mathrm{H}-6,12-14$ & 16.12 & 89.2 & 76.4 & $4 \mathrm{H}-5,130-132$ & 34.80 & 65.3 & 40.5 \\
\hline $2 \mathrm{H}-6,30-32$ & 16.30 & 87.5 & 76.8 & $4 \mathrm{H}-6,10-12$ & 35.10 & 66.2 & 51.2 \\
\hline $2 \mathrm{H}-6,54-56$ & 16.54 & 89.3 & 77.0 & $4 \mathrm{H}-6,30-32$ & 35.30 & 59.9 & 45.2 \\
\hline $2 \mathrm{H}-6,70-72$ & 16.70 & 88.4 & 75.0 & $4 \mathrm{H}-6,50-52$ & 35.50 & 63.4 & 43.8 \\
\hline $2 \mathrm{H}-6,88-90$ & 16.88 & 88.0 & 73.1 & $4 \mathrm{H}-6,70-72$ & 35.70 & 68.6 & 50.3 \\
\hline $2 \mathrm{H}-6,109-111$ & 17.09 & 88.3 & 73.9 & $4 \mathrm{H}-6,90-92$ & 35.90 & 67.9 & 47.5 \\
\hline $2 \mathrm{H}-6,132-134$ & 17.32 & 88.1 & 69.0 & $4 \mathrm{H}-6,110-112$ & 36.10 & 66.5 & 47.6 \\
\hline $2 \mathrm{H}-7,7-8$ & 17.57 & & & $4 \mathrm{H}-6,130-132$ & 36.30 & 72.2 & 47.8 \\
\hline $2 \mathrm{H}-7,27-29$ & 17.77 & 89.3 & 72.5 & $4 \mathrm{H}-7,10-12$ & 36.60 & 71.4 & 46.1 \\
\hline $2 \mathrm{H}-7,54-56$ & 18.04 & 86.5 & 68.3 & $4 \mathrm{H}-7,30-32$ & 36.80 & 71.9 & 46.8 \\
\hline $3 \mathrm{H}-1,10-12$ & 18.10 & 88.7 & 63.4 & $4 \mathrm{H}-7,50-52$ & 37.0 & 70.5 & 53.9 \\
\hline $3 \mathrm{H}-1,30-32$ & 18.30 & 83.3 & 63.1 & $4 \mathrm{H}-7,70-72$ & 37.20 & 72.3 & 58.9 \\
\hline $3 \mathrm{H}-1,52-54$ & 18.52 & 84.8 & 55.2 & $5 \mathrm{H}-1,10-12$ & 37.10 & 69.8 & 55.0 \\
\hline $3 \mathrm{H}-1,70-72$ & 18.70 & 84.6 & 63.5 & $5 \mathrm{H}-1,30-32$ & 37.30 & 68.8 & 51.0 \\
\hline $3 \mathrm{H}-1,90-92$ & 18.90 & 89.1 & 61.8 & $5 \mathrm{H}-1,50-52$ & 37.50 & 71.5 & 53.1 \\
\hline $3 \mathrm{H}-1,109-111$ & 19.09 & 87.3 & 69.7 & $5 \mathrm{H}-1,70-72$ & 37.70 & 71.6 & 48.6 \\
\hline $3 \mathrm{H}-1,131-132$ & 19.31 & 86.6 & 70.5 & $5 \mathrm{H}-1,90-92$ & 37.90 & 75.2 & 53.0 \\
\hline
\end{tabular}


Table 4 (continued).

\begin{tabular}{|c|c|c|c|c|c|c|c|}
\hline $\begin{array}{c}\text { Sample, } \\
\text { interval }(\mathrm{cm})\end{array}$ & $\begin{array}{l}\text { Depth } \\
\text { (mbsf) }\end{array}$ & $\begin{array}{c}\text { \% Carbonate content } \\
\text { Coarse fraction }\end{array}$ & $\begin{array}{l}\text { \% Carbonate content } \\
\text { Archive (bulk) }\end{array}$ & $\begin{array}{c}\text { Sample, } \\
\text { interval }(\mathrm{cm})\end{array}$ & $\begin{array}{l}\text { Depth } \\
\text { (mbsf) }\end{array}$ & $\begin{array}{l}\text { \% Carbonate content } \\
\text { Coarse fraction }\end{array}$ & $\begin{array}{l}\text { \% Carbonate content } \\
\text { Archive (bulk) }\end{array}$ \\
\hline $5 \mathrm{H}-1,110-112$ & 38.10 & 72.2 & 54.0 & $7 \mathrm{H}-1,50-52$ & 56.50 & 69.6 & 50.6 \\
\hline $5 \mathrm{H}-1,130-132$ & 38.30 & 76.0 & 55.5 & $7 \mathrm{H}-1,70-72$ & 56.70 & 68.5 & 65.4 \\
\hline $5 \mathrm{H}-2,10-12$ & 38.60 & 68.5 & 61.9 & $7 \mathrm{H}-1,90-92$ & 56.90 & 74.4 & 67.2 \\
\hline $5 \mathrm{H}-2,30-32$ & 38.80 & 69.5 & 57.2 & $7 \mathrm{H}-1,110-112$ & 57.10 & 62.3 & 61.3 \\
\hline $5 \mathrm{H}-2,50-52$ & 39.0 & 68.5 & 53.8 & $7 \mathrm{H}-1,130-132$ & 57.30 & 71.4 & 67.1 \\
\hline $5 \mathrm{H}-2,70-72$ & 39.20 & 67.4 & 49.0 & $7 \mathrm{H}-2,10-12$ & 57.60 & 73.0 & 62.2 \\
\hline $5 \mathrm{H}-2,90-92$ & 39.40 & 68.9 & 55.2 & $7 \mathrm{H}-2,30-32$ & 57.80 & 74.2 & 66.4 \\
\hline $5 \mathrm{H}-2,110-112$ & 39.60 & 69.1 & 54.8 & $7 \mathrm{H}-2,50-52$ & 58.0 & 70.0 & 65.1 \\
\hline $5 \mathrm{H}-2,130-132$ & 39.80 & 71.3 & 60.0 & $7 \mathrm{H}-2,70-72$ & 58.20 & 70.1 & 63.5 \\
\hline $5 \mathrm{H}-3,10-12$ & 40.10 & 67.3 & 54.4 & $7 \mathrm{H}-2,90-92$ & 58.40 & 72.0 & 63.6 \\
\hline $5 \mathrm{H}-3,30-32$ & 40.30 & 63.3 & 53.9 & $7 \mathrm{H}-2,110-112$ & 58.60 & 72.3 & 61.6 \\
\hline $5 \mathrm{H}-3,50-52$ & 40.50 & 63.4 & 49.5 & $7 \mathrm{H}-2,130-132$ & 58.80 & 68.7 & 63.3 \\
\hline $5 \mathrm{H}-3,70-72$ & 40.70 & 63.5 & 44.6 & $7 \mathrm{H}-3,10-12$ & 59.10 & 73.2 & 60.0 \\
\hline $5 \mathrm{H}-3,90-92$ & 40.90 & 64.6 & 44.5 & $7 \mathrm{H}-3,30-32$ & 59.30 & 65.8 & 59.6 \\
\hline $5 \mathrm{H}-3,110-112$ & 41.10 & 56.3 & 43.5 & $7 \mathrm{H}-3,50-52$ & 59.50 & 70.5 & 61.3 \\
\hline $5 \mathrm{H}-3,130-132$ & 41.30 & 67.7 & 48.3 & $7 \mathrm{H}-3,70-72$ & 59.70 & 69.0 & 59.4 \\
\hline $5 \mathrm{H}-4,10-12$ & 41.60 & 67.3 & 47.7 & $7 \mathrm{H}-3,90-92$ & 59.90 & 75.2 & 60.7 \\
\hline $5 \mathrm{H}-4,30-32$ & 41.80 & 68.7 & 50.5 & $7 \mathrm{H}-3,110-112$ & 60.10 & 70.3 & 58.1 \\
\hline $5 \mathrm{H}-4,50-52$ & 42.0 & 67.0 & 44.8 & $7 \mathrm{H}-3,130-132$ & 60.30 & 70.8 & 57.6 \\
\hline $5 \mathrm{H}-4,70-72$ & 42.20 & 68.3 & 49.5 & $7 \mathrm{H}-4,10-12$ & 60.60 & 70.4 & 61.9 \\
\hline $5 \mathrm{H}-4,90-92$ & 42.40 & 71.5 & 47.9 & $7 \mathrm{H}-4,30-32$ & 60.80 & 66.3 & 52.0 \\
\hline $5 \mathrm{H}-4,110-112$ & 42.60 & 72.0 & 48.0 & $7 \mathrm{H}-4,50-52$ & 61.0 & 72.3 & 60.3 \\
\hline $5 \mathrm{H}-4,130-132$ & 42.80 & 71.8 & 50.0 & $7 \mathrm{H}-4,70-72$ & 61.20 & 75.1 & 61.3 \\
\hline $5 \mathrm{H}-5,10-12$ & 43.10 & 68.9 & 48.9 & $7 \mathrm{H}-4,90-92$ & 61.40 & 74.8 & 60.8 \\
\hline $5 \mathrm{H}-5,30-32$ & 43.30 & 71.6 & 49.1 & $7 \mathrm{H}-4,110-112$ & 61.60 & 71.1 & 59.8 \\
\hline $5 \mathrm{H}-5,50-52$ & 43.50 & 72.5 & 49.6 & $7 \mathrm{H}-4,130-132$ & 61.80 & 74.1 & 61.7 \\
\hline $5 \mathrm{H}-5,70-72$ & 43.70 & 72.1 & 49.2 & $7 \mathrm{H}-5,10-12$ & 62.10 & 66.8 & 59.2 \\
\hline $5 \mathrm{H}-5,90-92$ & 43.90 & 76.2 & 54.4 & $7 \mathrm{H}-5,30-32$ & 62.30 & 72.8 & 58.0 \\
\hline $5 \mathrm{H}-5,110-112$ & 44.10 & 69.8 & 46.6 & $7 \mathrm{H}-5,50-52$ & 62.50 & 72.7 & 58.4 \\
\hline $5 \mathrm{H}-5,130-132$ & 44.30 & 75.5 & 55.8 & $7 \mathrm{H}-5,70-72$ & 62.70 & 72.4 & 53.8 \\
\hline $5 \mathrm{H}-6,10-12$ & 44.60 & 71.3 & 55.5 & $7 \mathrm{H}-5,90-92$ & 62.90 & 71.0 & 55.8 \\
\hline $5 \mathrm{H}-6,30-32$ & 44.80 & 75.3 & 56.7 & $7 \mathrm{H}-5,110-112$ & 63.10 & 70.2 & 58.2 \\
\hline $5 \mathrm{H}-6,50-52$ & 45.0 & 74.8 & 45.3 & $7 \mathrm{H}-6,10-12$ & 63.60 & 69.3 & 54.7 \\
\hline $5 \mathrm{H}-6,70-72$ & 45.20 & 72.7 & 54.1 & $7 \mathrm{H}-6,30-32$ & 63.80 & 71.1 & 49.6 \\
\hline $5 \mathrm{H}-6,90-92$ & 45.40 & 74.3 & 50.7 & $7 \mathrm{H}-6,50-52$ & 64.0 & 68.6 & 54.2 \\
\hline $5 \mathrm{H}-6,110-112$ & 45.60 & 72.2 & 46.1 & $7 \mathrm{H}-6,70-72$ & 64.20 & 67.3 & 56.0 \\
\hline $5 \mathrm{H}-6,130-132$ & 45.80 & 73.3 & 46.2 & $7 \mathrm{H}-6,90-92$ & 64.40 & 71.7 & 47.4 \\
\hline $5 \mathrm{H}-7,10-12$ & 46.10 & 57.5 & 38.6 & $7 \mathrm{H}-6,110-112$ & 64.60 & 71.2 & 51.8 \\
\hline $5 \mathrm{H}-7,30-32$ & 46.30 & 72.2 & 47.3 & $7 \mathrm{H}-6,130-132$ & 64.80 & 69.8 & 48.9 \\
\hline $5 \mathrm{H}-7,50-52$ & 46.50 & 69.7 & 48.1 & $7 \mathrm{H}-7,10-12$ & 65.10 & 71.0 & 49.1 \\
\hline $5 \mathrm{H}-7,70-72$ & 46.70 & 70.0 & 49.5 & $7 \mathrm{H}-7,30-32$ & 65.30 & 73.7 & 46.8 \\
\hline $6 \mathrm{H}-1,10-12$ & 46.60 & 70.5 & 45.7 & $7 \mathrm{H}-7,50-52$ & 65.50 & 69.4 & 45.4 \\
\hline $6 \mathrm{H}-1,30-32$ & 46.80 & 75.5 & 42.5 & $7 \mathrm{H}-7,70-72$ & 65.70 & 71.5 & 48.5 \\
\hline $6 \mathrm{H}-1,50-52$ & 47.0 & 71.4 & 41.9 & $8 \mathrm{H}-1,10-12$ & 65.60 & 75.9 & 66.7 \\
\hline $6 \mathrm{H}-1,70-72$ & 47.20 & 66.1 & 37.7 & $8 \mathrm{H}-1,30-32$ & 65.80 & 74.8 & 59.8 \\
\hline $6 \mathrm{H}-1,90-92$ & 47.40 & 67.2 & 37.8 & $8 \mathrm{H}-1,50-52$ & 66.0 & 71.0 & 55.9 \\
\hline $6 \mathrm{H}-1,110-112$ & 47.60 & 70.2 & 39.0 & $8 \mathrm{H}-2,10-12$ & 66.22 & 69.1 & 57.0 \\
\hline $6 \mathrm{H}-1,130-132$ & 47.80 & 70.7 & 38.9 & $8 \mathrm{H}-2,30-32$ & 66.42 & 65.3 & 54.0 \\
\hline $6 \mathrm{H}-2,10-12$ & 48.10 & 75.2 & 42.7 & $8 \mathrm{H}-2,50-52$ & 66.62 & 66.6 & 50.1 \\
\hline $6 \mathrm{H}-2,30-32$ & 48.30 & 73.5 & 39.4 & $8 \mathrm{H}-2,70-72$ & 66.82 & 64.8 & 42.5 \\
\hline $6 \mathrm{H}-2,50-52$ & 48.50 & 70.3 & 49.9 & $8 \mathrm{H}-2,90-92$ & 67.02 & 61.9 & 40.9 \\
\hline $6 \mathrm{H}-2,70-72$ & 48.70 & 73.3 & 40.3 & $8 \mathrm{H}-2,110-112$ & 67.22 & 54.9 & 40.0 \\
\hline $6 \mathrm{H}-2,90-92$ & 48.90 & 72.8 & 42.9 & $8 \mathrm{H}-2,130-132$ & 67.42 & 58.1 & 32.6 \\
\hline $6 \mathrm{H}-2,110-112$ & 49.10 & 72.7 & 43.6 & $8 \mathrm{H}-3,10-12$ & 67.72 & 59.5 & 31.5 \\
\hline $6 \mathrm{H}-2,130-132$ & 49.30 & 72.8 & 42.5 & $8 \mathrm{H}-3,30-32$ & 67.92 & 63.0 & 41.4 \\
\hline $6 \mathrm{H}-3,10-12$ & 49.60 & 73.4 & 54,8 & $8 \mathrm{H}-3,50-52$ & 68.12 & 70.4 & 44.5 \\
\hline $6 \mathrm{H}-3,30-32$ & 49.80 & 72.6 & 45.2 & $8 \mathrm{H}-3,70-72$ & 68.32 & 68.7 & 46.3 \\
\hline $6 \mathrm{H}-3,50-52$ & 50.0 & 71.5 & 57.8 & $8 \mathrm{H}-3,90-92$ & 68.52 & 68.4 & 55.4 \\
\hline $6 \mathrm{H}-3,70-72$ & 50.20 & 72.8 & 61.7 & $8 \mathrm{H}-3,110-112$ & 68.72 & 68.2 & 50.2 \\
\hline $6 \mathrm{H}-3,90-92$ & 50.40 & 72.6 & 60.2 & $8 \mathrm{H}-3,130-132$ & 68.92 & 70.2 & 48.0 \\
\hline $6 \mathrm{H}-3,110-112$ & 50.60 & 71.3 & 56.0 & $8 \mathrm{H}-4,10-12$ & 69.22 & 71.4 & 51.5 \\
\hline $6 \mathrm{H}-3,130-132$ & 50.80 & 71.5 & 64.2 & $8 \mathrm{H}-4,30-32$ & 69.42 & 68.9 & 51.3 \\
\hline $6 \mathrm{H}-4,10-12$ & 51.10 & 74.3 & 62.1 & $8 \mathrm{H}-4,50-52$ & 69.62 & 69.0 & 51.4 \\
\hline $6 \mathrm{H}-4,30-32$ & 51.30 & 72.4 & 62.9 & $8 \mathrm{H}-4,70-72$ & 69.82 & 65.9 & 43.4 \\
\hline $6 \mathrm{H}-4,50-52$ & 51.50 & 71.6 & 60.7 & $8 \mathrm{H}-4,90-92$ & 70.02 & 72.0 & 60.4 \\
\hline $6 \mathrm{H}-4,70-72$ & 51.70 & 71.5 & 62.1 & $8 \mathrm{H}-4,110-112$ & 70.22 & 69.0 & 47.0 \\
\hline $6 \mathrm{H}-4,90-92$ & 51.90 & 72.5 & 63.2 & $8 \mathrm{H}-4,130-132$ & 70.42 & 71.1 & 48.0 \\
\hline $6 \mathrm{H}-4,110-112$ & 52.10 & 73.2 & 62.6 & $8 \mathrm{H}-5,10-12$ & 70.72 & 67.7 & 48.1 \\
\hline $6 \mathrm{H}-4,130-132$ & 52.30 & 69.7 & 62.9 & $8 \mathrm{H}-5,30-32$ & 70.92 & 69.0 & 46.7 \\
\hline $6 \mathrm{H}-5,10-12$ & 52.600 & 71.4 & 62.6 & $8 \mathrm{H}-5,50-52$ & 71.12 & 66.7 & 44.7 \\
\hline $6 \mathrm{H}-5,30-32$ & 52.80 & 71.1 & 63.5 & $8 \mathrm{H}-5,70-72$ & 71.32 & 68.0 & 47.5 \\
\hline $6 \mathrm{H}-5,50-52$ & 53.0 & 72.4 & 64.2 & $8 \mathrm{H}-5,90-92$ & 71.52 & 71.6 & 48.0 \\
\hline $6 \mathrm{H}-5,70-72$ & 53.20 & 71.6 & 64.1 & $8 \mathrm{H}-5,110-112$ & 71.72 & 70.7 & 47.8 \\
\hline $6 \mathrm{H}-5,90-92$ & 53.40 & 67.4 & 66.5 & $8 \mathrm{H}-5,130-132$ & 71.92 & 79.1 & 41.9 \\
\hline $6 \mathrm{H}-5,110-112$ & 53.60 & 65.6 & 65.5 & $8 \mathrm{H}-6,10-12$ & 72.22 & 70.6 & 41.9 \\
\hline $6 \mathrm{H}-5,130-132$ & 53.80 & 63.1 & 67.0 & $8 \mathrm{H}-6,30-32$ & 72.42 & 68.4 & 47.0 \\
\hline $6 \mathrm{H}-6,10-12$ & 54.10 & 64.9 & 68.7 & $8 \mathrm{H}-6,50-52$ & 72.62 & 71.8 & 40.6 \\
\hline $6 \mathrm{H}-6,30-32$ & 54.30 & 60.9 & 68.7 & $8 \mathrm{H}-6,70-72$ & 72.82 & 69.5 & 50.3 \\
\hline $6 \mathrm{H}-6,50-52$ & 54.50 & 70.9 & 75.6 & $8 \mathrm{H}-6,90-92$ & 73.02 & 65.3 & 46.2 \\
\hline $6 \mathrm{H}-6,70-72$ & 54.70 & 70.1 & 71.7 & $8 \mathrm{H}-6,110-112$ & 73.22 & 63.2 & 45.9 \\
\hline $6 \mathrm{H}-6,90-92$ & 54.90 & 72.2 & 70.1 & $8 \mathrm{H}-6,130-132$ & 73.42 & 64.9 & 50.7 \\
\hline $6 \mathrm{H}-6,110-112$ & 55.10 & 70.0 & 70.0 & $8 \mathrm{H}-7,10-12$ & 73.72 & 72.4 & 50.4 \\
\hline $6 \mathrm{H}-6,130-132$ & 55.30 & 73.1 & 62.2 & $8 \mathrm{H}-7,30-32$ & 73.92 & 68.2 & 48.4 \\
\hline $7 \mathrm{H}-1,10-12$ & 56.10 & 73.0 & 67.7 & $8 \mathrm{H}-7,50-52$ & 74.12 & 67.0 & 47.6 \\
\hline $7 \mathrm{H}-1,30-32$ & 56.30 & 70.9 & 86.9 & $8 \mathrm{H}-7,70-72$ & 74.32 & 70.9 & 45.5 \\
\hline
\end{tabular}


Table 4 (continued).

\begin{tabular}{|c|c|c|c|}
\hline $\begin{array}{c}\text { Sample, } \\
\text { interval }(\mathrm{cm})\end{array}$ & $\begin{array}{l}\text { Depth } \\
\text { (mbsf) }\end{array}$ & $\begin{array}{c}\text { \% Carbonate content } \\
\text { Coarse fraction }\end{array}$ & $\begin{array}{l}\text { \% Carbonate content } \\
\text { Archive (bulk) }\end{array}$ \\
\hline $8 \mathrm{H}-7,90-92$ & 74.52 & 70.1 & 49.2 \\
\hline $8 \mathrm{H}-7,110-112$ & 74.72 & 69.0 & 48.6 \\
\hline $8 \mathrm{H}-7,130-132$ & 74.92 & 73.1 & 48.5 \\
\hline $9 \mathrm{H}-1,10-12$ & 75.10 & 72.5 & 50.0 \\
\hline $9 \mathrm{H}-1,30-32$ & 75.30 & 70.3 & 38.0 \\
\hline $9 \mathrm{H}-2,10-12$ & 75.45 & 66.1 & 41.5 \\
\hline $9 \mathrm{H}-2,30-32$ & 75.65 & 75.1 & 41.8 \\
\hline $9 \mathrm{H}-2,50-52$ & 75.85 & 68.5 & 43.4 \\
\hline $9 \mathrm{H}-2,70-72$ & 76.05 & 70.9 & 37.3 \\
\hline $9 \mathrm{H}-2,90-92$ & 76.25 & 71.3 & 41.2 \\
\hline $9 \mathrm{H}-2,110-112$ & 76.45 & 72.6 & 39.8 \\
\hline $9 \mathrm{H}-2,130-132$ & 76.65 & 62.8 & 42.0 \\
\hline $9 \mathrm{H}-3,30-32$ & 77.15 & 71.7 & 41.6 \\
\hline $9 \mathrm{H}-3,50-52$ & 77.35 & 74.6 & 42.7 \\
\hline $9 \mathrm{H}-3,70-72$ & 77.55 & 74.6 & 41.9 \\
\hline $9 \mathrm{H}-3,90-92$ & 77.75 & 70.3 & 43.9 \\
\hline $9 \mathrm{H}-3,110-112$ & 77.95 & 75.0 & 43.7 \\
\hline $9 \mathrm{H}-3,130-132$ & 78.15 & 71.6 & 44.2 \\
\hline $9 \mathrm{H}-4,10-12$ & 78.45 & 62.7 & 42.3 \\
\hline $9 \mathrm{H}-4,30-32$ & 78.65 & 69.7 & 43.9 \\
\hline $9 \mathrm{H}-4,50-52$ & 78.85 & 67.7 & 39.4 \\
\hline $9 \mathrm{H}-4,70-72$ & 79.05 & 72.5 & 34.0 \\
\hline $9 \mathrm{H}-4,90-92$ & 79.25 & 65.6 & 35.6 \\
\hline $9 \mathrm{H}-4,110-112$ & 79.45 & 58.6 & 35.6 \\
\hline $9 \mathrm{H}-4,130-132$ & 79.65 & 61.0 & 32.8 \\
\hline $9 \mathrm{H}-5,10-12$ & 79.95 & 77.1 & 61.3 \\
\hline $9 \mathrm{H}-5,30-32$ & 80.15 & 74.2 & 60.7 \\
\hline $9 \mathrm{H}-5,50-52$ & 80.35 & 76.2 & 57.2 \\
\hline $9 \mathrm{H}-5,70-72$ & 80.55 & 75.4 & 62.0 \\
\hline $9 \mathrm{H}-5,90-92$ & 80.75 & 71.6 & 62.4 \\
\hline $9 \mathrm{H}-5,110-112$ & 80.95 & 73.1 & 60.8 \\
\hline $9 \mathrm{H}-5,130-132$ & 81.15 & 73.9 & 60.7 \\
\hline $9 \mathrm{H}-6,10-12$ & 81.45 & 76.9 & 62.5 \\
\hline $9 \mathrm{H}-6,30-32$ & 81.65 & 75.9 & 63.8 \\
\hline $9 \mathrm{H}-6,50-52$ & 81.85 & 74.6 & 63.5 \\
\hline $9 \mathrm{H}-6,70-72$ & 82.05 & 75.5 & 63.7 \\
\hline $9 \mathrm{H}-6,90-92$ & 82.25 & 75.4 & 61.5 \\
\hline $9 \mathrm{H}-6,110-112$ & 82.45 & 61.6 & \\
\hline $9 \mathrm{H}-6,130-132$ & 82.65 & 74.6 & 64.7 \\
\hline $9 \mathrm{H}-7,10-12$ & 82.95 & 70.8 & 66.5 \\
\hline $9 \mathrm{H}-7,30-32$ & 83.15 & 73.8 & 67.4 \\
\hline $9 \mathrm{H}-7,50-52$ & 83.35 & 70.9 & 62.1 \\
\hline $9 \mathrm{H}-7,70-72$ & 83.55 & 71.4 & 60.0 \\
\hline $9 \mathrm{H}-7,90-92$ & 83.75 & 73.4 & 62.7 \\
\hline $9 \mathrm{H}-7,110-112$ & 83.95 & 73.3 & 64.3 \\
\hline $9 \mathrm{H}-7,130-132$ & 84.15 & 69.0 & 65.8 \\
\hline $9 \mathrm{H}-8,10-12$ & 84.45 & 69.1 & 58.1 \\
\hline $9 \mathrm{H}-8,30-32$ & 84.65 & 68.6 & \\
\hline $9 \mathrm{H}-8,50-52$ & 84.85 & 67.6 & 59.8 \\
\hline $9 \mathrm{H}-8,70-72$ & 85.05 & 72.4 & 64.6 \\
\hline $10 \mathrm{H}-1,10-12$ & 84.60 & 69.7 & \\
\hline $10 \mathrm{H}-1,30-32$ & 84.80 & 68.7 & 60.7 \\
\hline $10 \mathrm{H}-2,10-12$ & 85.0 & 68.4 & 64.1 \\
\hline $10 \mathrm{H}-2,30-32$ & 85.20 & 69.0 & 57.3 \\
\hline $10 \mathrm{H}-2,50-52$ & 85.40 & 67.4 & 61.6 \\
\hline $10 \mathrm{H}-2,70-72$ & 85.6 & 63.8 & 60.2 \\
\hline $10 \mathrm{H}-2,90-92$ & 85.8 & 63.7 & 60.5 \\
\hline $10 \mathrm{H}-2,110-112$ & 86.0 & 59.6 & 58.7 \\
\hline $10 \mathrm{H}-2,130-132$ & 86.20 & 67.1 & 58.4 \\
\hline $10 \mathrm{H}-3,10-12$ & 86.50 & 66.2 & 55.4 \\
\hline $10 \mathrm{H}-3,30-32$ & 86.70 & 63.9 & 56.3 \\
\hline $10 \mathrm{H}-3,50-52$ & 86.90 & 65.6 & 56.6 \\
\hline $10 \mathrm{H}-3,70-72$ & 87.10 & 62.9 & 51.1 \\
\hline $10 \mathrm{H}-3,90-92$ & 87.30 & 63.2 & 49.7 \\
\hline $10 \mathrm{H}-3,110-112$ & 87.50 & 67.0 & 49.6 \\
\hline $10 \mathrm{H}-3,130-132$ & 87.70 & 63.6 & 48.3 \\
\hline $10 \mathrm{H}-4,10-12$ & 88.0 & 66.6 & 47.8 \\
\hline $10 \mathrm{H}-4,30-32$ & 88.20 & 66.7 & 48.0 \\
\hline $10 \mathrm{H}-4,50-52$ & 88.40 & 64.7 & 47.2 \\
\hline $10 \mathrm{H}-4,70-72$ & 88.60 & 62.0 & 43.2 \\
\hline $10 \mathrm{H}-4,90-92$ & 88.80 & 65.0 & 43.1 \\
\hline $10 \mathrm{H}-4,110-112$ & 89.0 & 59.7 & 37.1 \\
\hline $10 \mathrm{H}-4,130-132$ & 89.20 & 57.1 & 30.8 \\
\hline $10 \mathrm{H}-5,10-12$ & 89.50 & 65.2 & 40.3 \\
\hline $10 \mathrm{H}-5,30-32$ & 89.70 & 55.2 & 29.0 \\
\hline $10 \mathrm{H}-5,50-52$ & 89.90 & 61.5 & 35.0 \\
\hline $10 \mathrm{H}-5,70-72$ & 90.10 & 56.2 & 30.8 \\
\hline $10 \mathrm{H}-5,90-92$ & 90.30 & 59.8 & 36.9 \\
\hline $10 \mathrm{H}-5,110-112$ & 90.50 & 67.0 & 35.9 \\
\hline $10 \mathrm{H}-6,10-12$ & 91.0 & 69.1 & 42.8 \\
\hline $10 \mathrm{H}-6,30-32$ & 91.20 & 73.3 & 48.6 \\
\hline $10 \mathrm{H}-6,50-52$ & 91.40 & 76.7 & 43.2 \\
\hline $10 \mathrm{H}-6,70-72$ & 91.60 & 81.9 & 48.6 \\
\hline $10 \mathrm{H}-6,90-92$ & 91.80 & 84.1 & 60.8 \\
\hline $10 \mathrm{H}-6,110-112$ & 92.0 & 74.1 & 52.1 \\
\hline
\end{tabular}

\begin{tabular}{|c|c|c|c|}
\hline $\begin{array}{c}\text { Sample, } \\
\text { interval }(\mathrm{cm})\end{array}$ & $\begin{array}{l}\text { Depth } \\
\text { (mbsf) }\end{array}$ & $\begin{array}{l}\text { \% Carbonate content } \\
\text { Coarse fraction }\end{array}$ & $\begin{array}{l}\text { \% Carbonate content } \\
\text { Archive (bulk) }\end{array}$ \\
\hline $10 \mathrm{H}-6,130-132$ & 92.20 & 77.3 & 45.6 \\
\hline $10 \mathrm{H}-7,10-12$ & 92.50 & 79.6 & 46.2 \\
\hline $10 \mathrm{H}-7,30-32$ & 92.70 & 83.1 & 47.0 \\
\hline $10 \mathrm{H}-7,50-52$ & 92.90 & 71.9 & 40.7 \\
\hline $10 \mathrm{H}-7,70-72$ & 93.10 & 74.1 & 44.6 \\
\hline $10 \mathrm{H}-7,90-92$ & 93.30 & 77.1 & 47.8 \\
\hline $10 \mathrm{H}-7,110-112$ & 93.50 & 78.0 & 47.6 \\
\hline $10 \mathrm{H}-7,130-132$ & 93.70 & 79.0 & 45.5 \\
\hline $10 \mathrm{H}-8,10-12$ & 94.0 & 84.4 & 47.5 \\
\hline $10 \mathrm{H}-8,30-32$ & 94.20 & 83.8 & 42.3 \\
\hline $10 \mathrm{H}-8,50-52$ & 94.40 & 78.0 & 44.0 \\
\hline $10 \mathrm{H}-8,70-72$ & 94.60 & 81.4 & 46.1 \\
\hline $11 \mathrm{H}-1,10-12$ & 94.10 & 63.0 & 43.7 \\
\hline $11 \mathrm{H}-2,10-12$ & 94.42 & 78.3 & 52.1 \\
\hline $11 \mathrm{H}-2,30-32$ & 94.62 & 83.3 & 50.1 \\
\hline $11 \mathrm{H}-2,50-52$ & 94.82 & 86.5 & 46.8 \\
\hline $11 \mathrm{H}-2,70-72$ & 95.02 & 84.7 & 45.4 \\
\hline $11 \mathrm{H}-2,90-92$ & 95.22 & 85.1 & 46.1 \\
\hline $11 \mathrm{H}-2,110-112$ & 95.42 & 82.2 & 55.0 \\
\hline $11 \mathrm{H}-2,130-132$ & 95.62 & 80.3 & 53.3 \\
\hline $11 \mathrm{H}-3,10-12$ & 95.92 & 85.0 & 52.7 \\
\hline $11 \mathrm{H}-3,30-32$ & 96.12 & 85.6 & 54.3 \\
\hline $11 \mathrm{H}-3,50-52$ & 96.32 & 83.2 & 51.6 \\
\hline $11 \mathrm{H}-3,70-72$ & 96.52 & 84.2 & 56.1 \\
\hline $11 \mathrm{H}-3,90-92$ & 96.72 & 84.3 & 54.7 \\
\hline $11 \mathrm{H}-3,110-112$ & 96.92 & 82.2 & 55.4 \\
\hline $11 \mathrm{H}-3,130-132$ & 97.12 & 80.8 & 52.6 \\
\hline $11 \mathrm{H}-4,10-12$ & 97.42 & 80.9 & 51.7 \\
\hline $11 \mathrm{H}-4,30-32$ & 97.62 & 85.6 & 52.7 \\
\hline $11 \mathrm{H}-4,50-52$ & 97.82 & 78.1 & 51.6 \\
\hline $11 \mathrm{H}-4,70-72$ & 98.02 & 82.0 & 49.2 \\
\hline $11 \mathrm{H}-4,90-92$ & 98.22 & 81.8 & 58.7 \\
\hline $11 \mathrm{H}-4,110-112$ & 98.42 & 79.4 & 59.5 \\
\hline $11 \mathrm{H}-4,130-132$ & 98.62 & 81.4 & 57.7 \\
\hline $11 \mathrm{H}-5,10-12$ & 98.92 & 81.2 & 58.5 \\
\hline $11 \mathrm{H}-5,30-32$ & 99.12 & 82.2 & 56.1 \\
\hline $11 \mathrm{H}-5,50-52$ & 99.32 & 81.3 & 60.4 \\
\hline $11 \mathrm{H}-5,70-72$ & 99.52 & 80.0 & 60.0 \\
\hline $11 \mathrm{H}-5,90-92$ & 99.72 & 79.9 & 56.9 \\
\hline $11 \mathrm{H}-5,110-112$ & 99.92 & 84.8 & 63.1 \\
\hline $11 \mathrm{H}-5,130-132$ & 100.12 & 82.0 & 61.9 \\
\hline $11 \mathrm{H}-6,10-12$ & 100.42 & 76.8 & 62.9 \\
\hline $11 \mathrm{H}-6,30-32$ & 100.62 & 76.2 & 65.8 \\
\hline $11 \mathrm{H}-6,50-52$ & 100.82 & 79.8 & 64.4 \\
\hline $11 \mathrm{H}-6,70-72$ & 101.02 & 76.5 & 62.6 \\
\hline $11 \mathrm{H}-6,90-92$ & 101.22 & 79.9 & 64.2 \\
\hline $11 \mathrm{H}-6,110-112$ & 101.42 & 79.0 & 65.3 \\
\hline $11 \mathrm{H}-6,130-132$ & 101.62 & 80.7 & 66.0 \\
\hline $11 \mathrm{H}-7,10-12$ & 101.92 & 81.5 & 66.0 \\
\hline $11 \mathrm{H}-7,30-32$ & 102.12 & 69.4 & 62.7 \\
\hline $11 \mathrm{H}-7,50-52$ & 102.32 & 76.6 & 63.1 \\
\hline $11 \mathrm{H}-7,70-72$ & 102.52 & 77.4 & 63.6 \\
\hline $11 \mathrm{H}-7,90-92$ & 102.72 & 71.4 & 61.0 \\
\hline $11 \mathrm{H}-7,110-112$ & 102.92 & 79.2 & 62.1 \\
\hline $11 \mathrm{H}-7,130-132$ & 103.12 & 78.8 & 63.3 \\
\hline $11 \mathrm{H}-8,10-12$ & 103.42 & 77.9 & 64.0 \\
\hline $11 \mathrm{H}-8,30-32$ & 103.62 & 78.1 & 64.3 \\
\hline $11 \mathrm{H}-8,50-52$ & 103.82 & 75.5 & 64.4 \\
\hline $11 \mathrm{H}-8,70-72$ & 104.02 & 68.5 & 50.8 \\
\hline $12 \mathrm{H}-1,10-12$ & 103.60 & 76.1 & 61.6 \\
\hline $12 \mathrm{H}-1,30-32$ & 103.08 & 74.1 & 50.3 \\
\hline $12 \mathrm{H}-1,50-52$ & 104.0 & 73.9 & 66.3 \\
\hline $12 \mathrm{H}-1,70-72$ & 104.20 & 67.6 & 50.0 \\
\hline $12 \mathrm{H}-1,90-92$ & 104.40 & & \\
\hline $12 \mathrm{H}-2,10-12$ & 105.10 & 73.1 & 50.7 \\
\hline $12 \mathrm{H}-2,30-32$ & 105.30 & 76.2 & 57.7 \\
\hline $12 \mathrm{H}-2,50-52$ & 105.50 & 76.8 & 55.7 \\
\hline $12 \mathrm{H}-2,70-72$ & 105.70 & 71.8 & 57.4 \\
\hline $12 \mathrm{H}-2,90-92$ & 105.90 & 76.5 & 59.7 \\
\hline $12 \mathrm{H}-2,110-112$ & 106.10 & 75.3 & 61.0 \\
\hline $12 \mathrm{H}-2,130-132$ & 106.30 & 81.0 & 66.4 \\
\hline $12 \mathrm{H}-3,10-12$ & 106.60 & 82.3 & 66.7 \\
\hline $12 \mathrm{H}-3,30-32$ & 106.80 & 78.1 & 64.4 \\
\hline $12 \mathrm{H}-3,50-52$ & 107.0 & 79.2 & 64.7 \\
\hline $12 \mathrm{H}-3,70-72$ & 107.20 & 79.5 & 64.9 \\
\hline $12 \mathrm{H}-3,83-85$ & 107.33 & 82.2 & 73.0 \\
\hline $12 \mathrm{H}-3,110-112$ & 107.60 & 76.5 & 64.7 \\
\hline $12 \mathrm{H}-4,10-12$ & 108.10 & 78.7 & 64.0 \\
\hline $12 \mathrm{H}-4,30-32$ & 108.30 & 78.9 & 72.9 \\
\hline $12 \mathrm{H}-4,50-52$ & 108.50 & 77.0 & 64.6 \\
\hline $12 \mathrm{H}-4,70-72$ & 108.70 & 77.7 & 70.2 \\
\hline $12 \mathrm{H}-4,90-92$ & 108.90 & 74.3 & 64.4 \\
\hline $12 \mathrm{H}-4,110-112$ & 109.10 & 78.3 & 68.1 \\
\hline $12 \mathrm{H}-4,130-132$ & 109.30 & 76.8 & 65.8 \\
\hline $12 \mathrm{H}-5,10-12$ & 109.60 & 81.3 & 61.0 \\
\hline
\end{tabular}


Table 4 (continued).

\begin{tabular}{|c|c|c|c|}
\hline $\begin{array}{c}\text { Sample, } \\
\text { interval }(\mathrm{cm})\end{array}$ & $\begin{array}{l}\text { Depth } \\
\text { (mbsf) }\end{array}$ & $\begin{array}{l}\text { \% Carbonate content } \\
\text { Coarse fraction }\end{array}$ & $\begin{array}{l}\text { \% Carbonate content } \\
\text { Archive (bulk) }\end{array}$ \\
\hline $12 \mathrm{H}-5,30-32$ & 109.80 & 77.7 & 62.3 \\
\hline $12 \mathrm{H}-5,50-52$ & 110.0 & 82.3 & 65.0 \\
\hline $12 \mathrm{H}-5,70-72$ & 110.20 & 76.7 & 63.0 \\
\hline $12 \mathrm{H}-5,90-92$ & 110.40 & 76.4 & 63.2 \\
\hline $12 \mathrm{H}-5,110-112$ & 110.60 & 77.1 & 63.8 \\
\hline $12 \mathrm{H}-5,128-130$ & 110.78 & 81.2 & 66.5 \\
\hline $13 \mathrm{H}-1,10-12$ & 111.10 & 81.0 & 72.3 \\
\hline $13 \mathrm{H}-1,30-32$ & 111.30 & 77.5 & 70.2 \\
\hline $13 \mathrm{H}-1,55-57$ & 111.55 & 75.7 & 70.6 \\
\hline $13 \mathrm{H}-2,10-12$ & 111.68 & 79.2 & 70.7 \\
\hline $13 \mathrm{H}-2,30-32$ & 111.88 & 80.0 & 71.7 \\
\hline $13 \mathrm{H}-2,50-52$ & 112.08 & 77.4 & 69.3 \\
\hline $13 \mathrm{H}-2,70-72$ & 112.28 & 80.7 & 69.4 \\
\hline $13 \mathrm{H}-2,88-90$ & 112.46 & 79.3 & 69.8 \\
\hline $13 \mathrm{H}-2,110-112$ & 112.68 & & \\
\hline $13 \mathrm{H}-2,130-132$ & 112.88 & 78.3 & 58.6 \\
\hline $13 \mathrm{H}-3,10-12$ & 113.13 & 75.6 & 69.0 \\
\hline $13 \mathrm{H}-3,30-32$ & 113.38 & 77.0 & 69.6 \\
\hline $13 \mathrm{H}-3,50-52$ & 113.58 & 78.3 & 65.6 \\
\hline $13 \mathrm{H}-3,70-72$ & 113.78 & 76.6 & 67.2 \\
\hline $13 \mathrm{H}-3,88-90$ & 113.96 & 76.1 & 63.8 \\
\hline $13 \mathrm{H}-3,110-112$ & 114.18 & 74.6 & 65.9 \\
\hline $13 \mathrm{H}-3,130-132$ & 114.38 & 77.4 & 71.0 \\
\hline $13 \mathrm{H}-4,10-12$ & 114.68 & 71.7 & 60.0 \\
\hline $13 \mathrm{H}-4,30-32$ & 114.88 & 72.3 & 64.0 \\
\hline $13 \mathrm{H}-4,50-52$ & 115.08 & 67.6 & 62.6 \\
\hline $13 \mathrm{H}-4,70-72$ & 115.28 & 72.1 & 71.1 \\
\hline $13 \mathrm{H}-4,88-90$ & 115.46 & 67.1 & 58.3 \\
\hline $13 \mathrm{H}-4,110-112$ & 115.68 & 72.4 & 54.7 \\
\hline $13 \mathrm{H}-4,130-132$ & 115.88 & 70.3 & 57.5 \\
\hline $13 \mathrm{H}-5,10-12$ & 116.18 & 60.4 & 54.6 \\
\hline $13 \mathrm{H}-5,30-32$ & 116.38 & 62.4 & 54.4 \\
\hline $13 \mathrm{H}-5,50-52$ & 116.58 & 64.3 & 56.3 \\
\hline $13 \mathrm{H}-5,70-72$ & 116.78 & 69.7 & 52.4 \\
\hline $13 \mathrm{H}-5,88-90$ & 116.96 & 64.0 & 48.2 \\
\hline $13 \mathrm{H}-5,110-112$ & 117.18 & 67.1 & 50.0 \\
\hline $13 \mathrm{H}-6,10-12$ & 117.68 & 73.0 & 40.7 \\
\hline $13 \mathrm{H}-6,30-32$ & 117.88 & 60.0 & 37.1 \\
\hline $13 \mathrm{H}-6,50-52$ & 118.08 & 62.1 & 39.6 \\
\hline $13 \mathrm{H}-6,70-72$ & 118.28 & 66.0 & 41.7 \\
\hline $13 \mathrm{H}-6,88-90$ & 118.46 & 69.0 & 44.5 \\
\hline $13 \mathrm{H}-6,110-112$ & 118.68 & 72.4 & 49.6 \\
\hline $13 \mathrm{H}-6,130-132$ & 118.88 & 70.9 & 50.0 \\
\hline $13 \mathrm{H}-7,10-12$ & 119.18 & 73.0 & 51.0 \\
\hline $13 \mathrm{H}-7,30-32$ & 119.38 & 73.3 & 56.0 \\
\hline $13 \mathrm{H}-7,50-52$ & 119.58 & 69.9 & 52.4 \\
\hline $13 \mathrm{H}-7,70-72$ & 119.78 & 73.6 & 54.2 \\
\hline $13 \mathrm{H}-7,88-90$ & 119.96 & 81.5 & 52.8 \\
\hline $13 \mathrm{H}-7,110-112$ & 120.18 & 71.7 & 49.8 \\
\hline $13 \mathrm{H}-7,130-132$ & 120.38 & 73.3 & 48.9 \\
\hline
\end{tabular}

Fig. 14). Upon entering the western Coral Sea, the SEC either diverges or is turned southward, depending on the austral season (Pickard et al., 1977). The southward-flowing component of the SEC becomes the East Australian Current (EAC) at about $20^{\circ} \mathrm{S}$ (Orme, 1973). Intensification of the northwest monsoon, during the austral summer, may account for the increased southerly currents near the Australian continental margin (Isern et al., this volume). Pickard et al. (1977) suggested that because of the build-up and sinking of watermasses associated with the SEC along today's Australian continental margin, currently, little significant upwelling is occurring.

Typical vertical profiles of temperature $(T)$, salinity $(S)$, density $(\sigma)$ and dissolved oxygen $\left(\mathrm{O}_{2}\right)$ for the western Coral Sea are illustrated in Figure 15 (Pickard et al., 1977). Although Figure 15 shows a set of typical physiochemical profiles from the western Coral Sea in $1200 \mathrm{~m}$ of water, we postulate that this model can be applied directly to the shallower upper slope setting at Hole 819A without significant modification. The important point to note is that the present-day position of Site 819 (water depth of $565.2 \mathrm{~m}$ ) is just below the main thermocline, the zone of rapid physiochemical oceanographic change (see Fig. 15).

In terms of watermasses, Site 819 today is situated between the cores of Subtropical Central Water (SCW; water depth of 50-250 m) and Antarctic Intermediate Water (AAIW; water depth of 650-1150 m), see Pickard et al. (1977) and Wyrtki (1962). Temperature ranges for these watermasses at the time are between $18^{\circ}-25^{\circ} \mathrm{C}$ and $4.2^{\circ}-6^{\circ} \mathrm{C}$ for SCW and AAIW, respectively (Wyrtki, 1962). Given the proximity of Site 819 to the core of AAIW, it is reasonable to assume that sedi- ments today are more likely to be influenced by AAIW during deposition than SCW. However, any changes in relative sea level in the past might have been accompanied by a change in the prevailing watermass.

\section{Paleodepth and Paleoceanography}

The dramatic upward decrease in sedimentation rates at Hole 819A and the overall increase in nannofossil percentages (Shipboard Scientific Party, 1991) below the hiatus (during sedimentological Units 5 through 2) suggest that the lower part of the section was at a shallower depth than the upper portion. Planktonic/benthic (P/B) foraminiferal ratio data (see Kroon et al., this volume), which are available for the upper $45 \mathrm{~m}$ of the sedimentary sequence at Hole $819 \mathrm{~A}$, also suggest shallower water depths during isotopic stages 15 to 28. Possibly, tectonic subsidence across the continental margin caused deepening at Site 819. Subsidence may not have been restricted to the continental margin, but also may have involved the adjoining Marion and Queensland plateaus. Subsidence across these ocean-circulation modulating bathymetric highs would enhance local AAIW circulation patterns and would enable these waters to encroach farther up the continental margin. With increasing water depth, a restructuring of the water column occurred, involving the intensification of both AAIW and SCW flow on the continental margin during the upper part of the sequence at Hole 819A. In addition, the regional spatial watermass distribution might have changed over time in a response to worldwide reorganization of watermasses (see below).

Assuming that Site 819 was at a shallower depth below the hiatus, the dominant watermass affecting sedimentation at Site 819 was most likely the SCW. This part of the record at Site 819 is dominated by predominantly fine-grained sediments (Fig. 9). High concentrations of fine-grained sediments indicate the absence of erosive currents and imply that SCW circulation was reasonably sluggish. The presence of SCW in this part of the record is also suggested by the low $\Delta \delta^{18} \mathrm{O}_{\mathrm{B}-\mathrm{P}}$ values, during stages 22 to 24 (Fig. 4), which imply little temperature difference between surface and bottom waters. Thus, cool AAIWs were unable to affect sedimentation at Site 819 during this period, as they had most likely been displaced farther down the slope by shallower water depths. However, at about stage 21 (i.e., close to the Brunhes/Matuyama boundary), the $\Delta \delta^{18} \mathrm{O}_{\text {B-p }}$ shows a marked increase in value. Higher $\Delta \delta^{18} \mathrm{O}_{\mathrm{B}-\mathrm{p}}$ values during stages 21 to 14 suggest the decoupling of surface and bottom water temperatures. Enhanced temperature stratification may have occurred in response to a change in the distribution of the watermasses, associated with the resurgence of cold AAIW flow at Hole 819A.

Throughout the upper part of the sequence at Hole 819A, we see a further continuation of the pattern of ocean-circulation intensification and reorganization, suggested during stages 21 to 14 . The upcore trend of increasing $\Delta \delta^{18} \mathrm{O}_{\mathrm{B}-\mathrm{P}}$ values, during the upper part of the sequence at Hole $819 \mathrm{~A}$, suggests the progressive decoupling of surface and bottom water temperatures. Furthermore, the planktonic foraminiferal oxygen isotopic record indicates (assuming that salinity changes are negligible) a gradual increase in mean surface-water temperatures above the hiatus. This trend might reflect depletion of ${ }^{16} \mathrm{O}$, associated with the progressive wedging southward of warm EAC surface waters. Increased southerly currents may reflect a longterm trend in the intensification of the northwest monsoon, in a similar manner to that outlined by Isern et al. (this volume).

The upper part of the sequence is also characterized by high $\mathrm{P} / \mathrm{B}$ ratios (Kroon et al., this volume), which possibly indicates higher productivity in the surface waters. This might reflect the increased influence of AAIW flow across the continental margin, although on a glacial/interglacial basis, no significant relationship can be seen. The presence of upwelling is difficult to substantiate using our foraminiferal isotopic data. Warm, well-stratified surface waters may have prevented AAIWs from reaching the ocean surface and only occasionally was the thermocline sufficiently eroded to allow these waters to "outcrop." However, the zone of mixing between the surface and 


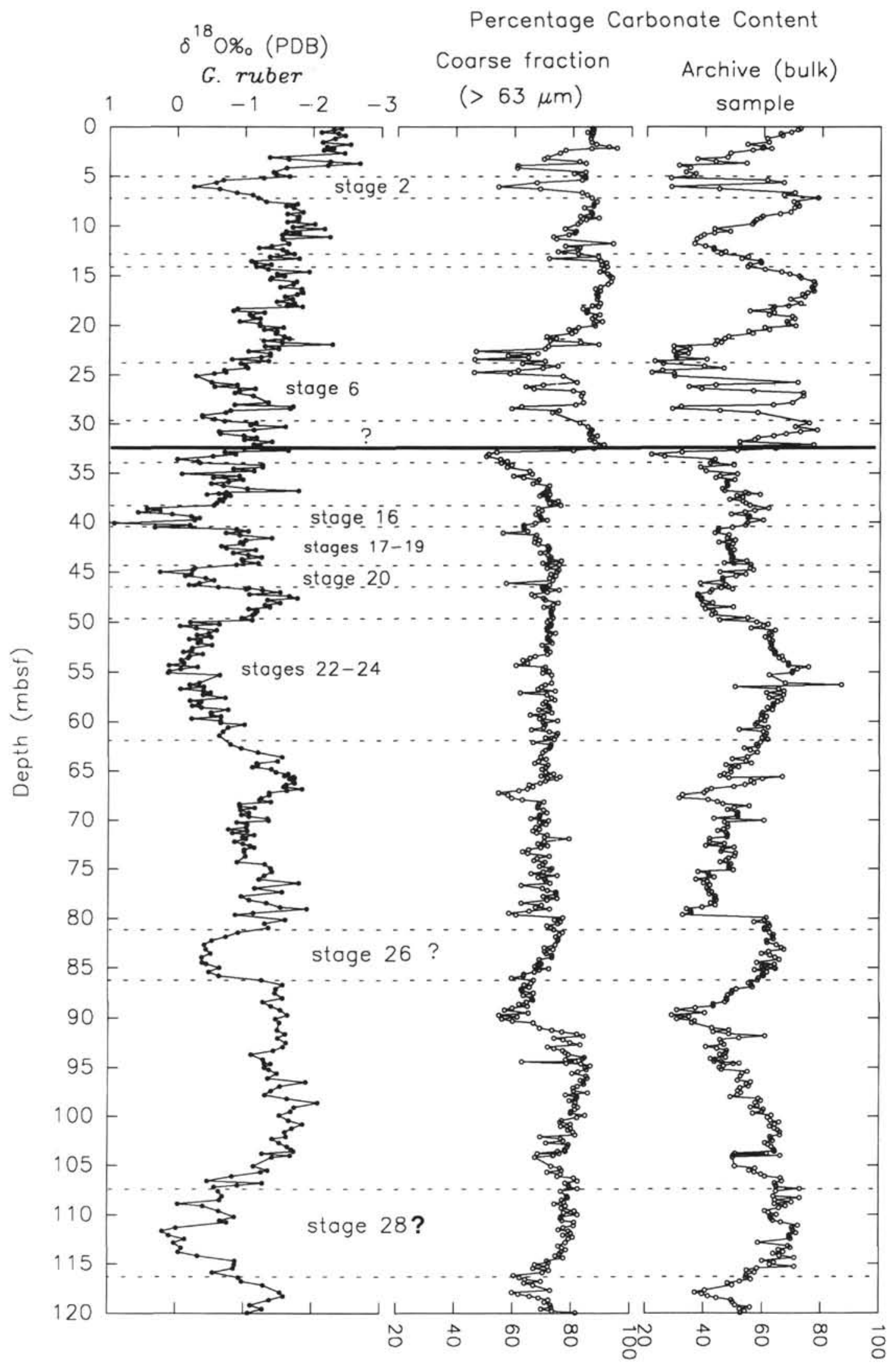

Figure 11. Percentage of carbonate content for coarse fraction $(>63 \mu \mathrm{m})$ and archive (bulk) samples and planktonic foraminiferal oxygen isotope record plotted vs. depth. 


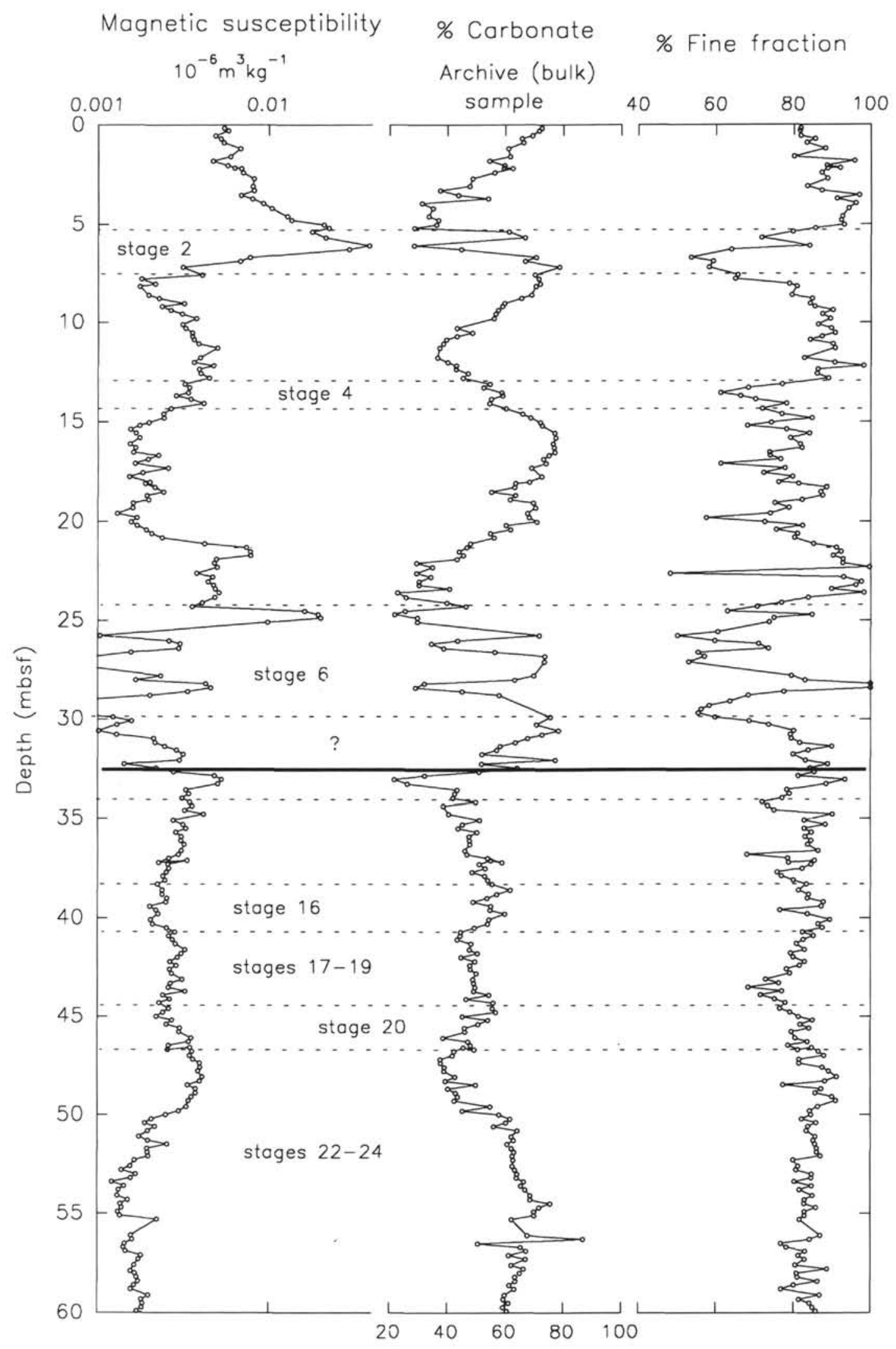

Figure 12. Magnetic susceptibility values, percentage of carbonate (archive sample) and percentage of fine fraction plotted vs. depth. Magnetic susceptibility has been plotted on a common logarithmic scale. 


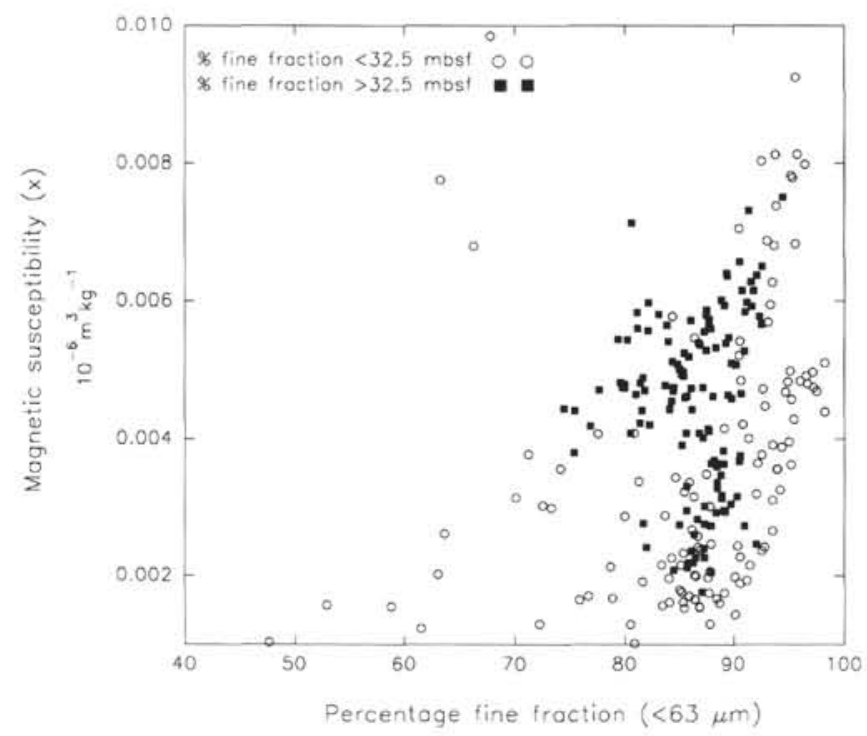

Figure 13. Scatter graph of magnetic susceptibility $(X)$ plotted vs. percentage of fine fraction $(<63 \mu \mathrm{m})$ above (open circles) and below (filled squares) 32.5 mbsf. The plot shows that no relationship occurred between the two variables below $35 \mathrm{mbsf}$ and a strong positive relationship above $35 \mathrm{mbsf}$ (Matthews, 1981). Magnetic susceptibility is plotted on a common logarithmic scale.

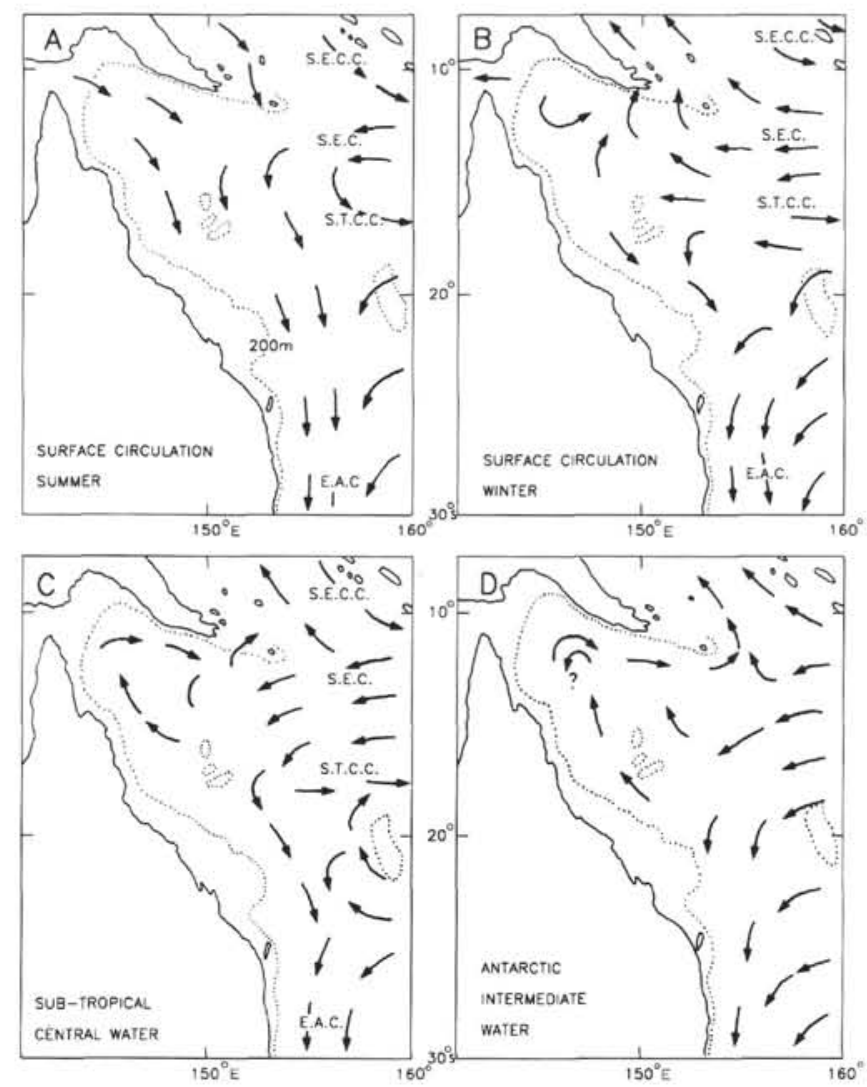

Figure 14. Surface and subsurface circulation patterns in austral summer and winter in the western Coral Sea (modified after Pickard et al., 1977). The South Equatorial Counter Current (SECC), the South Equatorial Current (SEC), the South Tropical Counter Current (STCC), and the East Australian Current (EAC) are illustrated in blocks A through D.

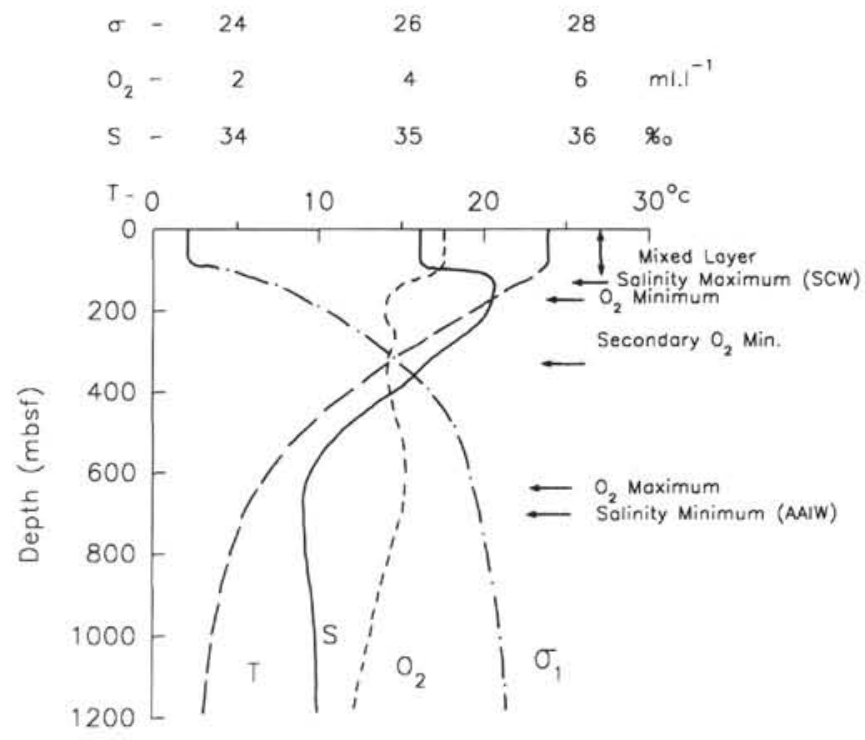

Figure 15. Typical vertical profiles of temperature $(T)$, salinity $(S)$, density $(\sigma)$ and dissolved oxygen $\left(\mathrm{O}_{2}\right)$ in $1200 \mathrm{~m}$ of water for the western Coral Sea (redrawn after Pickard et al., 1977). Note the changes in the structure of the thermocline/mixed layer in a water depth of between 200 and $550 \mathrm{~m}$.

subsurface watermasses may have become sufficiently shallow to facilitate nutrient transfer, thereby enabling the surface waters to support high abundances of planktonic foraminifers. Unfortunately, biogenic silica as a possible indicator of enhanced nutrient supply associated with upwelling (Codispoti, 1983) was not investigated in this study, and as such, our present data set is inconclusive. Further stable isotope and micropaleontological research will be required to substantiate the occurrence of periods of enhanced upwelling on the continental margin during the late Pleistocene at Hole 819A.

Most of our records suggest that a major change in the environment occurred across the major stratigraphic hiatus at Hole 819A. However, the foraminiferal record indicates that the change possibly occurred prior to the formation of the hiatus. Although some inconsistency can be seen among our records as to the exact timing of change, our data imply a significant reorganization of the climate and oceanography at or near the Brunhes/Matuyama boundary at Hole 819A.

The middle to late Pleistocene has been variously interpreted as a period of significant environmental change. Studies by Keany and Kennett (1972) indicated that mean temperatures were generally cooler during the Matuyama Chron than those during the Brunhes Chron. These results conflict with previous determinations of paleotemperature on the basis of radiolarian assemblages, which suggest mean warmer conditions throughout the Matuyama Chron (Hays, 1968; Bandy et al., 1971). Further, Keany and Kennett (1972) imply that climatic fluctuations during the Matuyama Chron were of lower magnitude than those in the Brunhes Chron. Although we did not see any convincing evidence of the temperature differences suggested by Keany and Kennett (1972), our records do suggest an intensification of glacial/interglacial climatic change above the hiatus. Kuijpers (1989) has interpreted the middle Pleistocene, between about 0.7 and $0.4 \mathrm{Ma}$ (early Brunhes), as a period of reduced AAIW flow at depths of its present depth stratum. However, Nelson et al. (1988) interpreted sedimentological data collected from the Challenger Plateau, South Tasman Sea, as indicating an increase in mean AAIW velocities after $0.5 \mathrm{Ma}$ (i.e, close to the timing of the major stratigraphic hiatus at Hole 819A). This suggests an absence of synchroneity in the timing of major oceanographic change throughout the world oceans. Jansen et al. (1986) reported a transition toward higher sea-surface temperatures (SSTs) and more "interglacial" conditions in the equatorial 
regions during the mid-Brunhes period (between 0.4 and $0.3 \mathrm{Ma}$.) and attributed the longterm climatic change in the Southern Ocean circulation to a worldwide reorganization of paleoclimate and paleoceanography, possibly linked to perturbation by the orbital eccentricity cycle. Thus, the records obtained from Hole $819 \mathrm{~A}$ indicate the occurrence of environmental change during the mid- to late Pleistocene. Our records, when combined with those from other Leg 133 holes, offer insight into the response of the Australian continental margin to fluctuations in paleoclimate and paleoceanography.

\section{ACKNOWLEDGMENTS}

We thank Tony Fallick and Terry Donnelly of the Scottish Universities Research Reactor Centre at East Kilbride for their assistance and endless patience with the "Prism," Ann Mennim and Kate Darling for their help with choosing foraminifers, and Mike Saunders and Tim Brand for their guidance during carbonate analysis. I.T. Alexander and D. Kroon gratefully acknowledge financial support from NERC (Grant No.GST/02/524).

\section{REFERENCES}

Agrawal, Y.C., McCave, I.N., and Riley, J.B., 1991. Laser diffraction size analysis. In Syvitski, J.P.M. (Ed.), Principles, Methods, and Application of Particle Size Analysis: (Cambridge Univ. Press), 368.

Bandy, O.L., Casey, R.E., and Wright, R.C., 1971. Late Neogene planktonic zonation, magnetic reversals and radiometric dates, Antarctic to tropics. Antarct. Res. Ser., 15:1-26.

Bloemendal, J., 1983. Paleoenvironmental implications of the magnetic characteristics of sediments from Deep Sea Drilling Project Site 514, southeast Argentine Basin. In Ludwig, W.J., Krasheninnikov, V.A., et al., Init. Repts. DSDP, 71 (Pt. 2): Washington (U.S. Govt. Printing Office), 1097-1108.

1989. Evidence for a change in the periodicity of tropical climate cycles at 2.4 Myr from whole-core magnetic susceptibility measurements. Nature, 342:897-900.

Bloemendal, J., Tauxe, L., Valet, J.-P., and Shipboard Scientific Party, 1988. High-resolution, whole-core magnetic susceptibility logs from Leg 108. In Ruddiman, W., Sarnthein, M., Baldauf, J., et al., Proc. ODP, Init. Repts., 108: College Station, TX (Ocean Drilling Program), 1005-1014.

Church, J.A., 1987. East Australian current adjacent to the Great Barrier Reef. Aust. J. Mar. Freshwater Res., 38:671-683.

Codispoti, L.A., 1983. On nutrient variability and sediments in upwelling regions. In Suess, E., and Thiede, J. (Eds.), Coastal Upwelling: Its Sediment Record: New York (Plenum), 125-145.

Craig, H., 1957. Isotopic standards for carbon and oxygen correction factors for mass spectrometric analysis of $\mathrm{CO}_{2}$. Geochim. Cosmochim. Acta, 12:133-149.

Dankers, P.H.M., 1978. Magnetic properties of dispersed natural iron-oxides of known grain-size [Ph.D. thesis]. Univ. of Utrecht.

Davies, P.J., Symonds, P.A., Feary, D.A., and Pigram, C.J., 1989. The evolution of the carbonate platforms of northeast Australia. In Crevello, P.D., Wilson, J.L., Sarg, J.F., Read, J.F. (Eds.), Controls on Carbonate Platform and Basin Development. Spec. Publ.-Soc. Econ. Paleontol. Mineral., 44:233-258.

Grimaldi, F.S., Shapiro, L., and Schnepfe, M., 1966. Determination of carbon dioxide in limestones and dolomite by acid-base titration. Geol. Surv. Prof. Pap. U.S., 550-B:B186-188.

Harris, P.T., Davies, P.J., and Marshall, J.F., 1990. Late Quaternary sedimentation on the Great Barrier Reef continental shelf and slope east of Townsville, Australia. Mar. Geol., 94:55-77.

Hays, J.D., 1968. Climatic record of late Cenozoic Antarctic Ocean sediments related to the record of world climate. In van Zinreren Bakker, E.M. (Ed.), Paleoecology of Africa and Antarctica: Cape Town (Balkema), 5:139-163.

Henshaw, P.C., Jr., and Merrill, R.T., 1980. Magnetic and chemical changes in marine sediments. Rev. Geophys. Space Phys., 18:483-504.
Hounslow, M.W., 1990. A magnetic susceptibility stratigraphy for Pleistocene and Pliocene sediments in the vicinity of the Barbados Ridge. In Moore, J.C., Mascle, A., et al., Proc. ODP, Sci. Results, 110: College Station, TX (Ocean Drilling Program), 365-377.

Jansen, J.H.F., Kuijpers, A., and Troelstra, S.R., 1986. A mid-Brunhes climatic event: long-term changes in global atmospheric and ocean circulation. Science, 232:619-622.

Karlin, R., and Levi, S., 1983. Diagenesis of magnetic minerals in recent hemipelagic sediments. Nature, 303:327-330.

Keany, J., and Kennett, J.P., 1972. Pliocene-early Pleistocene palaeoclimatic history recorded in Antarctic-Subantarctic deep-sea cores. Deep-Sea Res. Part A, 19:529-548.

Kent, D.V., 1982. Apparent correlation of paleomagnetic intensity and climatic records in deep-sea sediments. Nature, 299:538-539.

Kuijpers, A., 1986. Southern Ocean circulation and global climate in the Middle Pleistocene (early Brunhes). Palaeogeogr., Palaeoclimatol., Palaeoecol., 76:000-000.

Matthews, J.A., 1981. Quantitative and Statistical Approaches to Geography: A Practical Manual: Oxford (Pergamon Press), 138-154.

Morgan, G.E., and Smith, P.P.K., 1981. Transmission electron microscope and rock magnetic investigations of remanance carriers in a Precambrian metadolerite. Earth Planet. Sci. Lett., 53:226-240.

Nelson, C.S., Hendy, C.H., and Dudley, W.C., 1986. Quaternary isotope stratigraphy of Hole 593, Challenger Plateau, South Tasman Sea: preliminary observations based on foraminifers and calcareous nannofossils. In Kennett, J.P., von der Borch, C.C. et al., Init. Repts. DSDP, 90 (Pt. 2): Washington (U.S. Govt. Printing Office), 1413-1424.

Oldfield, F., and Robinson, S.G., 1985. Geomagnetism and palaeoclimate. In Tooley, M.J., and Sheail, G.M. (Eds.), The Climatic Scene: London (Allen and Unwin), 186-205.

Orme, G.R., 1973. The Coral Sea Plateau: a major reef province. In Jones, O.A., and Endean, R. (Eds.), Biology and Geology of Coral Reefs: New York (Academic Press), 267-306.

Pickard, G.L., Donguy, J.R., Henin, C., and Rougerie, F., 1977. A Review of the Physical Oceanography of the Great Barrier Reef and western Coral Sea. Aust. Inst. Mar. Sci. Monogr. Ser., 2.

Raymo, M.E., Ruddiman, W.F., Shackleton, N.J., and Oppo, D.W., 1990. Evolution of global ice volume and Atlantic-Pacific $\delta^{13} \mathrm{C}$ gradients over the last 2.5 m.y. Earth Planet. Sci. Lett., 97:353-368.

Robinson, S.G., 1986. The late Pleistocene paleoclimatic record of North Atlantic deep-sea sediments revealed by mineral-magnetic measurements. Phys. Earth Planet. Inter., 42:22-47.

Shipboard Scientific Party, 1991 Site 819. In Davies, P.J., McKenzie, J.A., Palmer-Julson, A., et al., Proc. ODP, Init. Repts., 133: College Station, TX (Ocean Drilling Program).

Tarling, D.H., 1983. Palaeomagnetism, Principles and Applications in Geology, Geophysics and Archaeology: New York (Chapman and Hall).

Thompson, P.R., Allan, W.H.B., Duplessy, J.-C., and Shackleton, N.J., 1979 Disappearance of pink-pigmented Globigerinoides ruber at $120,000 \mathrm{yr}$ BP in the Indian and Pacific oceans. Nature, 280:554-558.

Thompson, R., 1979. Palaeomagnetic correlation and dating. In Berglund, B.E. (Ed.), Palaeohydrological Changes in the Temperate Zone in the Last 150,000 Years. Subproject B: Lake and Mire Environments (Vol. 2): Dep. Quat. Geol., 39-59.

1986. Modelling magnetization data using SIMPLEX:Phys. Earth. Planet. Int., 42:113-127.

Wyrtki, K., 1962. The subsurface water masses in the western South Pacific Ocean. Aust. J. Mar. Freshwater Res., 13:18-47.

\footnotetext{
- Abbreviations for names of organizations and publication titles in ODP reference lists follow the style given in Chemical Abstracts Service Source Index (published by American Chemical Society).
}

Date of initial receipt: 30 April 1992

Date of acceptance: 12 February 1993

Ms 133SR-224 